\title{
Universality of one-dimensional Fermi systems, I. Response functions and critical exponents.
}

\author{
G. Benfatto ${ }^{1}$ \\ P. Falco ${ }^{2}$ \\ V. Mastropietro ${ }^{3}$
}

March 22, 2022

\begin{abstract}
The critical behavior of one-dimensional interacting Fermi systems is expected to display universality features, called Luttinger liquid behavior. Critical exponents and certain thermodynamic quantities are expected to be related among each others by model-independent formulas. We establish such relations, the proof of which has represented a challenging mathematical problem, for a general model of spinning fermions on a one dimensional lattice; interactions are short ranged and satisfy a positivity condition which makes the model critical at zero temperature. Proofs are reported in two papers: in the present one, we demonstrate that the zero temperature response functions in the thermodynamic limit are Borel summable and have anomalous power-law decay with multiplicative logarithmic corrections. Critical exponents are expressed in terms of convergent expansions and depend on all the model details. All results are valid for the special case of the Hubbard model.
\end{abstract}

\section{Main Results}

\section{$1.1 \quad$ Introduction}

The charge carriers in metals are described by a gas of non relativistic quantum particles (fermions). In the absence of interactions their thermodynamic properties can be computed and provide a good understanding of the physical properties of several systems. However, the free gas description fails in many important cases and cannot explain phenomena, such as the superconductivity, which are of the greatest importance both from the applicative and theoretical point of view, providing a dramatic manifestation of quantum physics at the macroscopic scales.

The analytic study of the properties of interacting fermions at zero temperature and in the thermodynamic limit is an extremely difficult task, and in several important cases even a convincing qualitative picture is lacking. From the point of view of mathematical physics, to this date only in two cases the ground state properties of a gas of weakly interacting fermions in dimensions greater than one has been constructed with full mathematical rigor by using Renormalization Group methods coming from Constructive Quantum Field Theory: the case of non symmetric Fermi surface [1] and the case of fermions on the honeycomb lattice at halffilling [2]. Both cases are rather special, as the interaction does not qualitatively modify the physical properties even at zero temperature. The rigorous study of an interacting model with a non trivial behavior in two or three dimensions is still a challenging problem.

The situation is analytically more accessible for a one dimensional gas of interacting fermions, where the interaction produces a number of remarkable effects which are believed to have a

\footnotetext{
${ }^{1}$ Dipartimento di Matematica, Università di Roma "Tor Vergata", 00133 Roma, Italy.

${ }^{2}$ Department of Mathematics, California State university, Northridge, CA 91330.

${ }^{3}$ Dipartimento di Matematica F.Enriquez, Università di Milano, Via Saldini 50, Milano, Italy.
} 
counterpart even at higher dimensions, in some special cases [3. In addition to this role as a benchmark for higher dimensions, the rapid progress of technology is producing materials which are a physical realization of such systems. One dimensional fermion gases have been extensively analyzed in the physical literature in the last forty years by a variety of methods. Their behavior is radically different with respect to the free gas, and the physical picture which is commonly accepted is the so-called Luttinger liquid conjecture proposed by Haldane [4] (extending previous ideas by Kadanoff [5], and Luther and Peschel [6]): according to such conjecture, the low energy properties at zero temperature of a wide class of interacting many body fermion systems in one dimension are characterized by: a) anomalous dimensions, that is the presence of critical model dependent exponents in the correlations decay; b) universality, in the sense that the exponents and other thermodynamic quantities verify a set of model independent relations.

The universality property is particularly remarkable; in experiments we have a poor knowledge of the microscopic parameters, but the validity of the universal relations imply that one can predict exact and parameter-free relations among exponents which could be experimentally measured. The universal Luttinger liquid relations are verified in a special solvable spinless models, the Luttinger model, which is the prototype of Luttinger liquid behavior. Its exact solvability relies on the absence of the spin and on the linear dispersion relation of the fermions, two features allowing for the mapping in a model of free bosons by Mattis and Lieb 7. Non relativistic fermions have a non linear dispersion relation, but Haldane 8 provided arguments that, at least in some cases, the relations can be true even if the mapping to free bosons is lost. The conjecture was partially verified in a solvable model, the XYZ spin chain, which is equivalent to a system of spinless fermions on the lattice with a nearest neighbor interaction, whose ground state energy can be computed by the Bethe ansatz. The solvability relies however on special and non generic peculiarity of certain models, and traditional methods cannot say too much on the validity of the Luttinger liquid relations in generic non solvable models. For instance, Field theoretic Renormalization Group analysis 9 confirms the existence of anomalous exponents and shows that the contributions from the non-linear part of the dispersion relation is irrelevant in the Renormalization Group sense; however such irrelevant terms, which contribute to the exponents, are simply discarded in this approach so that nothing can be concluded on the validity of the universal relations. In recent times indeed a caveat for a not too extensive application of the Luttinger liquid picture has been emerged; in particular, it appeared that non linear bands surely affect the finite temperature and the dynamical properties, see e.g. 10. In addition, the possibility of different physical properties, at least for the finite temperature and the dynamical properties, between integrable and non integrable 1D models has been extensively investigated, especially regarding the conduction properties.

All the above considerations surely provide a strong motivation for a mathematical proof of the Luttinger liquid relations, and we will provide here such a proof for a standard (generically non solvable) model of a gas of spinning fermions on a one dimensional lattice with a short range interaction satisfying a positivity condition, to be defined later. We will call such system extended Hubbard model, as it reduces to the (solvable) Hubbard model in the special case of ultralocal interaction. As it will clear by our analysis, the proof will be independent from the details of the model considered, and it could be generalized to a wider class of systems. However, for definiteness and sake of simplicity, we will not try to consider the most general class of models. We use non-perturbative Renormalization Group methods implemented with Ward Identities at each Renormalization Group step, using a technique introduced in [11, 12]. Such methods have provided for the first time the self-consistent construction (that is, without resorting to properties found by exact solutions as was done in previous works [13]) of an interacting non solvable many body model with a non trivial behavior (that is, where the interaction produces a different behavior with respect to the free case); namely a system of weakly interacting spinless fermions in one dimension. Subsequently, by such methods the Luttinger liquid relations [14, 15] for this spinless case were proven. 
The analysis of the spinning case, which is discussed in the present work, present considerable new difficulties. Indeed the fact that the inclusion of the spin in one dimensional physics produces new phenomena, such as the spin charge separation, logarithmic corrections and the possibility of metal-insulator transitions, is well known in the physical literature, see e.g. 3, 16, 17. The approximations leading to the solvable Luttinger model in the spinless case, namely the linearization of the dispersion relation, in the spinning case lead to a non solvable model. Power law decay with anomalous exponents are found only for repulsive interactions and in the non half filled band case; besides the power law decay has multiplicative logarithmic corrections. Despite such features, we can establish for the first time the validity of a number of universal Luttinger liquid relations connecting the exponents and other thermodynamical quantities in a generic non solvable model of $1 \mathrm{D}$ spinning fermions on a lattice.

The proof is split in two papers. In the present one we present the Renormalization Group construction of the model, which allows us to analyze the asymptotic behavior of the correlations, to prove the existence of critical exponents and logarithmic corrections and to establish their Borel summability, assuming the validity of a property called asymptotic vanishing of the Beta function. The exponents and the other physical quantities are expressed by sophisticated expansions, and while the validity of the universal relations can be checked at lowest order, a direct verification at all orders from the expansions look essentially impossible. Therefore, in the subsequent paper [18, we introduce an effective model verifying a several extra symmetries (which are only asymptotic in the lattice model); by fine tuning of its parameters one can show that its exponents are the same as in the original model, and on the other hand such symmetries imply Ward Identities, from which the asymptotic vanishing of the Beta function and the universal relations can be derived. This method is a way to implement the concept of emerging symmetries in a rigorous mathematical setting.

\subsection{Extended Hubbard Model and Physical Observables}

The Hamiltonian of a standard model of spinning fermions on a one dimensional lattice (also called extended Hubbard model) is

$$
H=-\frac{1}{2} \sum_{\substack{x \in \mathcal{C} \\ s= \pm}}\left(a_{x, s}^{+} a_{x+1, s}^{-}+a_{x, s}^{+} a_{x-1, s}^{-}\right)+\bar{\mu} \sum_{\substack{x \in \mathcal{C} \\ s= \pm}} a_{x, s}^{+} a_{x, s}^{-}+\lambda \sum_{\substack{x, y \in \mathcal{C} \\ s, s^{\prime}= \pm}} v_{L}(x-y) a_{x, s}^{+} a_{x, s}^{-} a_{y, s^{\prime}}^{+} a_{y, s^{\prime}}^{-}
$$

where

1. $\mathcal{C}=\{-[L / 2] \leq x \leq[(L-1) / 2]\}$ is a one dimensional lattice of step 1 and $L$ sites;

2. $a_{x, s}^{ \pm}$are fermion creation and annihilation operators at site $x$ with spin $s$, verifying

$$
\left\{a_{x, s}^{+}, a_{x^{\prime}, s^{\prime}}^{-}\right\}=\delta_{x, x^{\prime}} \delta_{x, x^{\prime}} \quad\left\{a_{x, s}^{+}, a_{x^{\prime}, s^{\prime}}^{+}\right\}=\left\{a_{x, s}^{+}, a_{x^{\prime}, s^{\prime}}^{+}\right\}=0
$$

and such that $a_{-[L / 2], s}^{ \pm}=a_{-[(L-1) / 2]+1, s}^{ \pm}$(periodic boundary conditions);

3. $v_{L}(x)$ is a function on $\mathbb{Z}$, periodic of period $L$, such that $v_{L}(x)=v(x)$ for $x \in \mathcal{C}, v(x)$ being an even function on $\mathbb{Z}$ satisfying the short range condition $|v(x)| \leq C e^{-\kappa|x|}$;

4. $-\bar{\mu} \in(-1,+1)$ is the chemical potential.

The results of this paper are only valid under the following condition on the potential $v(x)$, that we call the positivity condition:

$$
\lambda \hat{v}(2 \arccos (\bar{\mu})) \geq 0
$$

The model is $S U(2)$ symmetric, as the Hamiltonian is invariant under the transformation $a_{x, s}^{ \pm} \rightarrow \sum_{s^{\prime}} M_{s, s^{\prime}} a_{x, s^{\prime}}^{ \pm}$, with $M \in S U(2)$, and includes the standard (exactly solvable, [19]) and 
the U-V Hubbard models, corresponding to the interactions $\lambda v(x-y)=U \delta_{x, y}$ and $\lambda v(x-y)=$ $U \delta_{x, y}+\frac{1}{2} V \delta_{|x-y|, 1}$, respectively: in the former case the positive condition is $U \geq 0$.

We consider the operators $a_{\mathbf{x}, s}^{ \pm}=e^{x_{0} H} a_{x}^{ \pm} e^{-H x_{0}}$, with

$$
\mathbf{x}=\left(x, x_{0}\right), \quad 0 \leq x_{0}<\beta
$$

for some $\beta>0$ ( $\beta^{-1}$ is the temperature); on $x_{0}$ antiperiodic boundary conditions are imposed, that is, if $a_{\mathbf{x}, s}^{ \pm}=a_{x, x_{0}, s}^{ \pm}$, then $a_{x, \beta, s}^{ \pm}=-a_{x, 0, s}^{ \pm}$. Defining

$$
\langle\cdot\rangle_{L, \beta}:=\frac{\operatorname{Tr}\left[e^{-\beta H} \cdot\right]}{\operatorname{Tr}\left[e^{-\beta H}\right]}
$$

and $\langle\cdot\rangle_{L, \beta}^{T}$ the corresponding truncated expectation, the energy of the thermal ground state is

$$
E(\lambda):=-\lim _{\beta \rightarrow \infty} \lim _{L \rightarrow \infty}(L \beta)^{-1} \log \operatorname{Tr}\left[e^{-\beta H}\right],
$$

The Schwinger functions are defined as

$$
S_{n}^{\beta, L}\left(\mathbf{x}_{1}, \varepsilon_{1}, s_{1} ; \ldots ; \mathbf{x}_{n}, \varepsilon_{n}, s_{n}\right)=\left\langle\mathbf{T}\left\{a_{\mathbf{x}_{1}, s_{1}}^{\varepsilon_{1}} \cdots a_{\mathbf{x}_{n}, s_{n}}^{\varepsilon_{1}}\right\}\right\rangle_{\beta, L}^{T}
$$

where $\mathbf{T}$ is the operator of time ordering, acting on a product of fermion fields as:

$$
\mathbf{T}\left(a_{\mathbf{x}_{1}, s_{1}}^{\varepsilon_{1}} \ldots a_{\mathbf{x}_{n}, s_{n}}^{\varepsilon_{n}}\right)=(-1)^{\pi} a_{\mathbf{x}_{\pi(1)}, \sigma_{\pi(1)}}^{\varepsilon_{\pi(1)}} \ldots a_{\mathbf{x}_{\pi(n)}, s_{\pi(n)}}^{\varepsilon_{\pi(n)}}
$$

where $\pi$ is a permutation of $\{1, \ldots, n\}$, chosen in such a way that $x_{\pi(1) 0} \geq \cdots \geq x_{\pi(n) 0}$, and $(-1)^{\pi}$ is its sign. [If some of the time coordinates are equal each other, the arbitrariness of the definition is solved by ordering each set of operators with the same time coordinate so that creation operators precede the annihilation operators.] Note that $S_{n}^{\beta, L}$ is $L$-periodic in each $x_{i}$, $\beta$-antiperiodic in $x_{0, i}$ and is identically zero if $\sum_{i=1}^{n} \varepsilon_{i} \neq 0$.

We will introduce also the densities $\rho_{\mathbf{x}}^{\alpha}$ :

$$
\begin{aligned}
& \rho_{\mathbf{x}}^{C}=\sum_{s= \pm} a_{\mathbf{x}, s}^{+} a_{\mathbf{x}, s}^{-} \\
& \rho_{\mathbf{x}}^{S_{i}}=\sum_{s, s^{\prime}= \pm} a_{\mathbf{x}, s}^{+} \sigma_{s, s^{\prime}}^{(i)} a_{\mathbf{x}, s^{\prime}}^{-} \\
& \rho_{\mathbf{x}}^{S C}=\frac{1}{2} \sum_{\substack{s= \pm \varepsilon= \pm}} s a_{\mathbf{x}, s}^{\varepsilon} a_{\mathbf{x},-s}^{\varepsilon} \\
& \rho_{\mathbf{x}}^{T C_{i}}=\frac{1}{2} \sum_{\substack{s, s^{\prime}= \pm \varepsilon= \pm}} a_{\mathbf{x}, s}^{\varepsilon} \widetilde{\sigma}_{s, s^{\prime}}^{(i)} a_{\mathbf{x}+\mathbf{e}, s^{\prime}}^{\varepsilon}, \quad \mathbf{e}=(1,0)
\end{aligned}
$$

where $i=1,2,3$ and

$$
\begin{array}{rlrl}
\sigma^{(1)} & =\left(\begin{array}{ll}
0 & 1 \\
1 & 0
\end{array}\right) & \sigma^{(2)}=\left(\begin{array}{cc}
0 & -i \\
i & 0
\end{array}\right) & \sigma^{(3)}=\left(\begin{array}{cc}
1 & 0 \\
0 & -1
\end{array}\right) \\
\widetilde{\sigma}^{(1)}=\left(\begin{array}{ll}
1 & 0 \\
0 & 0
\end{array}\right) & \widetilde{\sigma}^{(2)}=\left(\begin{array}{ll}
0 & 1 \\
1 & 0
\end{array}\right) & \widetilde{\sigma}^{(3)}=\left(\begin{array}{ll}
0 & 0 \\
0 & 1
\end{array}\right)
\end{array}
$$

The response functions are defined by the following truncated correlations:

$$
\Omega_{\alpha, \beta, L}(\mathbf{x}-\mathbf{y}):=\left\langle\mathbf{T} \rho_{\mathbf{x}}^{\alpha} \rho_{\mathbf{y}}^{\alpha}\right\rangle_{\beta, L}^{T}:=\left\langle\mathbf{T} \rho_{\mathbf{x}}^{\alpha} \rho_{\mathbf{y}}^{\alpha}\right\rangle_{\beta, L}-\left\langle\rho_{\mathbf{x}}^{\alpha}\right\rangle_{\beta, L}\left\langle\rho_{\mathbf{y}}^{\alpha}\right\rangle_{\beta, L}
$$

where, if $O_{\mathbf{x}}$ is quadratic in the fermion operators, $\mathbf{T} O_{\mathbf{x}} O_{\mathbf{y}}=O_{\mathbf{x}} O_{\mathbf{y}}$ if $x_{0} \geq y_{0}$ and $O_{\mathbf{y}} O_{\mathbf{x}}$ if $x_{0} \leq y_{0}$. If $\mathbf{x}-\mathbf{y}=(\xi, \tau)$, the response functions are defined in $(-L, L) \times[-\beta, \beta]$ and are 
$\beta$-periodic in $\tau$ and $L$-periodic in $\xi$. If $F_{\beta, L}$ is any function of this type, we define its Fourier transform as

$$
\hat{F}_{\beta, L}(\mathbf{p})=\int_{-\frac{\beta}{2}}^{\frac{\beta}{2}} d x_{0} \sum_{x \in \mathcal{C}} e^{i \mathbf{p x}} F_{\beta, L}(\mathbf{x})
$$

where $\mathbf{p}=\left(p, p_{0}\right)$, with $p=\frac{2 \pi}{L} n,-[L / 2] \leq n \leq[(L-1) / 2]$ and $p_{0} \in \frac{2 \pi}{\beta} \mathbb{Z}$.

In the following we will be interested in the zero temperature limit of the Schwinger functions and response functions, calculated in the thermodynamic limit. We shall denote these functions by the same symbols, without the $\beta$ and $L$ labels; for example, we shall write: $\lim _{\beta \rightarrow \infty} \lim _{L \rightarrow \infty} \hat{\Omega}_{L, \beta, \alpha}(\mathbf{p}) \equiv \hat{\Omega}_{\alpha}(\mathbf{p})$. Note that the thermodynamic limit $L \rightarrow \infty$ is taken before the zero temperature limit $\beta \rightarrow \infty$; this allows us to derive properties of the thermal ground state. To shorten the notation, in the following we shall use the definition

$$
\lim _{\beta, L \rightarrow \infty} \equiv \lim _{\beta \rightarrow \infty} \lim _{L \rightarrow \infty}
$$

\subsection{The non interacting case}

In absence of interaction, the Hamiltonian is

$$
H_{0}=-\frac{1}{2} \sum_{\substack{x \in \mathcal{C} \\ s= \pm}}\left(a_{x, s}^{+} a_{x+1, s}^{-}+a_{x, s}^{+} a_{x-1, s}^{-}\right)+\mu \sum_{\substack{x \in \mathcal{C} \\ s= \pm 1}} a_{x, s}^{+} a_{x, s}^{-}
$$

Being $H_{0}$ quadratic, the $2 n$-point (not truncated) correlation functions of the $a_{\mathbf{x}, s}^{ \pm}$operators satisfy the Wick rule, i.e.

$$
\begin{aligned}
& \left\langle\mathbf{T}\left\{a_{\mathbf{x}_{1}, s_{1}}^{-} \cdots a_{\mathbf{x}_{n}, s_{n}}^{-} a_{\mathbf{y}_{1}, s_{1}^{\prime}}^{+} \cdots a_{\mathbf{y}_{n}, s_{n}^{\prime}}^{+}\right\}\right\rangle_{\beta, L}=\operatorname{det} G, \\
& G_{i j}=\delta_{s_{i}, s_{j}^{\prime}}\left\langle\mathbf{T}\left\{a_{\mathbf{x}_{i}, s_{i}}^{-} a_{\mathbf{y}_{j}, s_{j}^{\prime}}^{+}\right\}\right\rangle_{\beta, L} .
\end{aligned}
$$

Therefore, all the $n$-point Schwinger function $S_{n}^{\beta, L}\left(\mathbf{x}_{1}, \varepsilon_{1}, s_{1} ; \ldots ; \mathbf{x}_{n}, \varepsilon_{n}, \sigma_{n}\right)$ (truncated by definition) are identically zero for any $n>2$, and, in order to construct the whole set of response functions, it is enough to compute the 2-point function $g^{\beta, L}(\mathbf{x}-\mathbf{y})=\left\langle\mathbf{T}\left\{a_{\mathbf{x}, s}^{-} a_{\mathbf{y}, s}^{+}\right\}\right\rangle_{\beta, L}$, which is equal to

$$
\begin{aligned}
& g^{\beta, L}(\mathbf{x}-\mathbf{y})=\frac{\operatorname{Tr}\left[e^{-\beta H_{0}} \mathbf{T}\left(a_{\mathbf{x}}^{-} a_{\mathbf{y}}^{+}\right)\right]}{\operatorname{Tr}\left[e^{-\beta H_{0}}\right]}=\frac{1}{L} \sum_{k \in \mathcal{D}_{L}} e^{-i k(x-y)} \hat{g}^{\beta, L}\left(k, x_{0}-y_{0}\right)= \\
& =\frac{1}{L} \sum_{k \in \mathcal{D}_{L}} e^{-i k(x-y)}\left\{\frac{e^{-\left(x_{0}-y_{0}\right) e(k)}}{1+e^{-\beta e(k)}} \mathbf{I}\left(x_{0}-y_{0}>0\right)-\frac{e^{-\left(\beta+x_{0}-y_{0}\right) e(k)}}{1+e^{-\beta e(k)}} \mathbf{I}\left(x_{0}-y_{0} \leq 0\right)\right\}
\end{aligned}
$$

where $\mathbf{I}(t)$ is the indicator function, $\mathcal{D}_{L}=\{(2 \pi n) / L, n \in \mathbb{Z}\}$ and

$$
e(k)=\mu-\cos k
$$

The function $\hat{g}^{\beta, L}(k, \tau)$ is defined only for $-\beta<\tau<\beta$, but we can extend it periodically over the whole real axis. This periodic extension is smooth in $\tau$ for $\tau \neq n \beta, n \in \mathbb{Z}$, but has a jump discontinuity at $\tau=n \beta$ equal to $(-1)^{n}$. It follows that $g^{\beta, L}\left(x, x_{0}\right)$ is smooth in $x_{0}$ for $x_{0} \neq n \beta, n \in \mathbb{Z}$, with a jump discontinuity at $x_{0}=n \beta$ equal to $(-1)^{n} \delta_{x, y}$; hence, it is discontinuous only at $\mathbf{x}=(0, n \beta)$

The function $\hat{g}^{\beta, L}(k, \tau)$ is antiperiodic in $\tau$ of period $\beta$; hence its Fourier series is of the form

$$
\hat{g}^{\beta, L}(k, \tau)=\frac{1}{\beta} \sum_{k_{0}=\frac{2 \pi}{\beta}\left(n_{0}+\frac{1}{2}\right)} \hat{g}^{\beta, L}\left(k_{0}, k\right) e^{-i k_{0} \tau}
$$


with

$$
\hat{g}^{\beta, L}(\mathbf{k})=\int_{0}^{\beta} d \tau e^{i \tau k_{0}} \frac{e^{-\tau e(k)}}{1+e^{-\beta e(k)}}=\frac{1}{-i k_{0}+e(k)}
$$

It is a classical result that, because of the jump discontinuities, this series is not absolutely convergent; however, if we call $g_{N}^{\beta, L}(k, \tau)$ the sum over the terms with $\left|k_{0}\right| \leq N, g_{N}^{\beta, L}(k, \tau)$ is pointwise convergent and the limit is given by $\hat{g}^{\beta, L}(k, \tau)$ at the continuity points, while at the discontinuities it is given by the mean of the right and left limits. Hence, if $\mathbf{x}-\mathbf{y} \neq(0, n \beta)$, we can write

$$
g^{\beta, L}(\mathbf{x}-\mathbf{y})=\lim _{N \rightarrow \infty} \frac{1}{\beta L} \sum_{k \in \mathcal{D}_{L, \beta},\left|k_{0}\right| \leq N} \frac{e^{-i \mathbf{k}(\mathbf{x}-\mathbf{y})}}{-i k_{0}+e(k)}
$$

with $\mathcal{D}_{L, \beta}:=\mathcal{D}_{L} \times \mathcal{D}_{\beta}, \mathcal{D}_{L}:=\frac{2 \pi}{L} \mathcal{C}, \mathcal{D}_{\beta}:=\frac{2 \pi}{\beta}\left(\mathbb{Z}+\frac{1}{2}\right)$.

It is convenient, for reasons that will appear clear below, to slightly modify the representation (1.19) in the following way. Let us take a smooth even compact support function $\chi_{0}(t)$, equal to 1 for $|t|<1$ and equal to 0 if $|t| \geq \gamma$, for a given scaling parameter $\gamma>1$, fixed throughout the paper. In App. $₫$ we prove that (1.19) is completely equivalent to the representation

$$
g^{\beta, L}(\mathbf{x}-\mathbf{y})=\lim _{M \rightarrow \infty} \frac{1}{\beta L} \sum_{\mathbf{k} \in D_{\beta, L}} \chi_{0}\left(\gamma^{-M} k_{0}\right) \frac{e^{-i \mathbf{k}(\mathbf{x}-\mathbf{y})}}{-i k_{0}+e(k)}
$$

In particular, the above equality is not true for $\mathbf{x}-\mathbf{y}=(0, n \beta)$, where the propagator is equal, to $g^{L, \beta}\left(0,0^{-}\right) \rightarrow_{\beta, L \rightarrow \infty}-p_{F} / \pi$ while the r.h.s. is equal to

$$
\frac{g^{\beta, L}\left(0,0^{+}\right)+g^{\beta, L}\left(0,0^{-}\right)}{2} \rightarrow_{\beta, L \rightarrow \infty}-\frac{p_{F}}{\pi}+\frac{1}{2}
$$

where $p_{F}=\cos ^{-1} \mu$ is the Fermi momentum. The Fermi momentum appears in the period of the oscillations of the large distance behavior of the propagator; for $|\mathbf{x}|$ large,

$$
\lim _{\beta, L \rightarrow \infty} g^{\beta, L}(\mathbf{x}) \equiv g(\mathbf{x}) \sim \sum_{\omega= \pm} \frac{e^{-i \omega p_{F} x}}{v_{F} x_{0}+i \omega x}, \quad v_{F} \equiv \sin p_{F}
$$

where $\sim$ means up to faster decaying terms; $v_{F}$ is usually called the Fermi velocity.

\subsection{The interacting case}

The first step of our construction consists in computing the large distance behavior at zero temperature and in the thermodynamic limit of the two-points Schwinger function and of the response functions, proving the presence of anomalous critical exponents and logarithmic corrections.

Theorem 1.1 Let us consider the Schwinger and response functions, (1.7) and (1.10), with Hamiltonian (1.1). If $\bar{\mu} \neq 0$ and $\hat{v}(2 \arccos (\bar{\mu}))>0$, there exists $\lambda_{0}>0$ such that, if $0 \leq \lambda \leq \lambda_{0}$, it is possible to find a continuous function $p_{F} \equiv p_{F}(\bar{\mu}, \lambda)=\arccos (\bar{\mu})+O(\lambda)$ verifying the conditions

$$
p_{F} \neq 0, \pi / 2, \pi, \quad \hat{v}\left(2 p_{F}\right)>0
$$

such that, setting $v_{F}=\sin p_{F}$ and defining

$$
\begin{aligned}
\widetilde{\mathbf{x}}:=\left(x, v_{F} x_{0}\right), & L(\mathbf{x}):=1+b \lambda \widehat{v}\left(2 \bar{p}_{F}\right) \log |\mathbf{x}|, \quad b=2\left(\pi \sin p_{F}\right)^{-1} \\
\bar{\Omega}_{0}(\mathbf{x}):=\frac{x_{0}^{2}-x^{2}}{x_{0}^{2}+x^{2}}, & \bar{S}_{0}(\mathbf{x}):=\frac{v_{F} x_{0} \cos p_{F}-x \sin p_{F}}{|\widetilde{\mathbf{x}}|}
\end{aligned}
$$


in the limit $\beta, L \rightarrow \infty$ (1.12), the large $|\mathbf{x}|$ asymptotic behavior of the two-points Schwinger function $S_{2}(\mathbf{x}) \equiv S_{2}(\mathbf{x},-, s ; \mathbf{0},+, s)$ is of the form

$$
S_{2}(\mathbf{x}) \sim\left[\bar{S}_{0}(\mathbf{x})+R_{2}(\mathbf{x})\right] \frac{L(\mathbf{x})^{\zeta_{z}}}{|\widetilde{\mathbf{x}}|^{1+\eta}}
$$

where $R_{2}(\mathbf{x})$ is a continuous function of $\lambda$ and $\mathbf{x}$, such that, for any $\vartheta<1$ and a suitable positive constants $C_{\vartheta},\left|R_{2}(\mathbf{x})\right| \leq C_{\vartheta} \lambda^{1-\vartheta}$; the sign $\sim$ means up to terms bounded by $C|\mathbf{x}|^{-1-\vartheta}$. Moreover, the large $|\mathbf{x}|$ asymptotic behavior of the correlations is of the form

$$
\begin{aligned}
\text { for } \quad \alpha=C, S_{i} & \Omega_{\alpha}(\mathbf{x}) \sim \frac{\bar{\Omega}_{0}(\widetilde{\mathbf{x}})+R_{\alpha}(\mathbf{x})}{\pi^{2}|\widetilde{\mathbf{x}}|^{2}}+\cos \left[2 p_{F} x\right] \frac{L(\mathbf{x})^{\zeta_{\alpha}}}{\pi^{2}|\widetilde{\mathbf{x}}|^{2 X_{\alpha}}}\left[1+\widetilde{R}_{\alpha}(\mathbf{x})\right] \\
\text { for } \quad \alpha=S C & \Omega_{\alpha}(\mathbf{x}) \sim-\left[\bar{\Omega}_{0}(\widetilde{\mathbf{x}})+\widetilde{R}_{\alpha}(\mathbf{x})\right] \cos \left(2 p_{F} x\right) \frac{L(\mathbf{x})^{\widetilde{\zeta}_{\alpha}}}{\pi^{2}|\widetilde{\mathbf{x}}|^{2 \widetilde{X}_{\alpha}}}-\frac{1}{\pi^{2}} \frac{L(\mathbf{x})^{\zeta_{\alpha}}}{|\widetilde{\mathbf{x}}|^{2 X_{\alpha}}}\left[1+R_{\alpha}(\mathbf{x})\right] \\
\text { for } \quad \alpha=T C_{i} & \Omega_{\alpha}(\mathbf{x}) \sim-\frac{v_{F}^{2}}{\pi^{2}} \frac{L(\mathbf{x})^{\zeta_{\alpha}}}{|\widetilde{\mathbf{x}}|^{2 X_{\alpha}}}\left[1+R_{\alpha}(\mathbf{x})\right]
\end{aligned}
$$

with the functions $R_{\alpha}(\mathbf{x})$ and $\widetilde{R}_{\alpha}(\mathbf{x})$ having the same properties of $R_{2}(\mathbf{x})$; the sign $\sim$ means up to terms bounded by $C|\mathbf{x}|^{-2-\vartheta}$.

The critical exponents $\eta$ and $X_{\alpha}$ are continuous functions of $\lambda$, such that $\eta(0)=X_{\alpha}(0)-1=0$ and $\eta / \lambda^{2}>0$, while the exponents $\widetilde{\zeta}_{S C}$ and $\zeta_{\alpha}$ of the logarithmic corrections could also depend on $\mathbf{x}$ (we can not exclude it), but satisfy the bounds $\left|\widetilde{\zeta}_{S C}\right| \leq C \lambda$ and $\left|\zeta_{\alpha}-\bar{\zeta}_{\alpha}\right| \leq C \lambda$, for a suitable constant $C$, with

$$
\bar{\zeta}_{z}=0, \quad \bar{\zeta}_{C}=-\frac{3}{2}, \quad \bar{\zeta}_{S_{i}}=\frac{1}{2}, \quad \bar{\zeta}_{S C}=-\frac{3}{2}, \quad \bar{\zeta}_{T C_{i}}=\frac{1}{2}
$$

Finally, given $\delta \in(0, \pi / 2)$, there exists $\varepsilon \equiv \varepsilon(\delta)>0$, such that the free energy, the two-points Schwinger functions and the density correlations are analytic in the set

$$
D_{\varepsilon, \delta}=\{\lambda \in \mathbb{C}: 0<|\lambda|<\varepsilon,|\operatorname{Arg} \lambda|<\pi-\delta\}
$$

continuous in the closure $\bar{D}_{\varepsilon, \delta}$ and Borel summable in $\lambda=0$.

This Theorem will be proved in 93 . It is completely based on the multi-scale analysis of the Grassmannian functional representation of the model, which is discussed in $\$ 2$. In this analysis we choose, for technical reasons, to fix the Fermi momentum $p_{F}$ of the interacting model by adding to the chemical potential a finite counterterm $\nu\left(\lambda, p_{F}\right)$, which is uniquely determined by the condition that the multi-scale expansion is well defined; in $\$ 2.9$ we prove that the relation between $p_{F}$ and $\nu$ can be inverted, so determining the function $p_{F}(\bar{\mu}, \lambda)$.

\section{Remarks.}

1. If $\bar{\mu}=0$, a different behavior is expected, as proved in [19] for the (exactly solvable) Hubbard model.

2. In the free $\lambda=0$ case the response functions decay for large distance with power law of exponent equal to 2 . The interaction partially removes such degeneracy by producing anomalous exponents which are (in general) non trivial functions of the coupling.

3. While the presence of non universal exponents in the model (1.1) is a common feature with the Luttinger model, both in the spinless [7] and spinning case [20, the presence of logarithmic corrections is a striking difference. Such corrections remove the degeneracy in the response of charge and spin densities, present in the spinning Luttinger model. 
4. The exponents of the non oscillating part of charge or spin density correlations are the same as in the free case; also logarithmic corrections are excluded.

5. In the Luttinger model the exponents, as function of the coupling, are analytic in a complex disk around $\lambda=0$, both in the spinless and spinning case. This property is valid also for the a general spinless model with short range interaction [13, 21, but in the present spinning case the perturbative expansion in $\lambda=0$ is only Borel summable.

6. Our analysis could be extended to the generic $2 l$-point Schwinger function, by using the same strategy used in $\S 2.3$ of [22] to analyze the corresponding tree expansion in the case of the Thirring model.

\section{RG Analysis for the extended-Hubbard Model}

\subsection{Functional integral representation}

The analysis of the Hubbard model correlations is done by a rigorous implementation of the RG techniques. To begin with, we need a functional integral representation of the model, because the RG techniques are optimized for that.

We find convenient (even if not necessary) to fix the value of the singularities of two-point function Fourier transform $\hat{S}_{2}^{\beta, L}(\mathbf{k})$ (that is, of the Fermi momentum $p_{F}$ ) by writing the chemical potential $\bar{\mu}$ in (1.1) in the form

$$
\bar{\mu}=\mu+\nu^{\beta, L}(\lambda), \quad \mu=\cos p_{F, L}
$$

where $p_{F, L}=\frac{2 \pi}{L}\left(n_{F}+\frac{1}{2}\right)$, with $n_{F}=\left[\left(p_{F} L\right) /(2 \pi)\right]$; then we show that it is possible to choose $\nu^{\beta, L}(\lambda)$, uniquely up to corrections of order $\min \left\{L^{-1}, \beta^{-1}\right\}$, so that the interacting Fermi momentum is indeed $p_{F}$, in the limit $\beta, L \rightarrow \infty$ (1.12). Our results can be translated in the form of Theorem 1.1. because we can show that the equation (2.1) has a unique solution $p_{F}=p_{F}(\bar{\mu}, \lambda)$ in a right interval of $\lambda=0$, small enough (how small depending on $p_{F}$ ).

The choice, at finite $L$, of $p_{F, L}$ in place of $p_{F}$ is motivated by technical reasons, see $\$ 2.4$ below; this choice does not affect the infinite volume limit, since it changes $\mu$ for terms of order $1 / L$ and $\nu^{\beta, L}(\lambda)$ is defined up to terms of the same order. by

The main object we will study is the functional $\mathcal{W}(J, \eta)$ (depending on $M, L$ and $\beta$ ), defined

$$
\begin{gathered}
\mathcal{W}(J, \eta)=-L \beta e_{C}+r_{C} \int d \mathbf{x} J_{\mathbf{x}}^{C}+\log \int P(d \psi) \exp \left\{-\mathcal{V}^{(M)}(\psi)+\mathcal{B}^{(M)}(\psi, J, \eta)\right\} \\
-\mathcal{V}^{(M)}(\psi)=-\mathcal{V}(\psi)-\nu \mathcal{N}(\psi)-\nu_{C} \mathcal{N}(\psi) \\
\mathcal{B}^{(M)}(\psi, J, \eta)=\sum_{\alpha} \int d \mathbf{x} J_{\mathbf{x}}^{\alpha} \rho_{\mathbf{x}}^{\alpha}+\sum_{s} \int d \mathbf{x}\left[\eta_{\mathbf{x}, s}^{+} \psi_{\mathbf{x}, s}^{-}+\psi_{\mathbf{x}, s}^{+} \eta_{\mathbf{x}, s}^{-}\right]
\end{gathered}
$$

where $\psi_{\mathbf{x}, s}^{ \pm}$and $\eta_{\mathbf{x}, s}^{ \pm}$are Grassmann variables and the fermion density operators $\rho_{\mathbf{x}}^{\alpha}$ are defined as in (1.9), with $\psi_{\mathbf{x}, s}^{ \pm}$in place of $a_{\mathbf{x}, s}^{ \pm}, J_{\mathbf{x}}^{\alpha}$ are commuting variables, $\int d \mathbf{x}$ is a short form for $\sum_{x \in \mathcal{C}} \int_{-\beta / 2}^{\beta / 2} d x_{0}, P(d \psi)$ is a Grassmann-valued Gaussian measure in the field variables $\psi_{\mathbf{x}, s}^{ \pm}$with covariance (the free propagator) given by

$$
\begin{aligned}
& \int P(d \psi) \psi_{\mathbf{x}, s}^{\varepsilon} \psi_{\mathbf{y}, s^{\prime}}^{\varepsilon}=0, \quad \int P(d \psi) \psi_{\mathbf{x}, s}^{-} \psi_{\mathbf{y},-s}^{+}=0 \\
& \bar{g}_{\beta, L, M}(\mathbf{x}-\mathbf{y}):=\int P(d \psi) \psi_{\mathbf{x}, s}^{-} \psi_{\mathbf{y}, s}^{+}=\frac{1}{\beta L} \sum_{\mathbf{k} \in \mathcal{D}_{L, \beta}} \frac{\chi_{0}\left(\gamma^{-M} k_{0}\right) e^{-i \mathbf{k}(\mathbf{x}-\mathbf{y})}}{-i k_{0}+\left(\cos p_{F, L}-\cos k\right)}
\end{aligned}
$$


In the above formulae, $\chi_{0}(t)$ and $\mathcal{D}_{L, \beta}$ are defined as in (1.20),

$$
\mathcal{V}(\psi)=\lambda \sum_{s, s^{\prime}= \pm} \int d \mathbf{x} d \mathbf{y} \psi_{\mathbf{x}, s}^{+} \psi_{\mathbf{x}, s}^{-} v(\mathbf{x}-\mathbf{y}) \psi_{\mathbf{y}, s^{\prime}}^{+} \psi_{\mathbf{y}, s^{\prime}}^{-}
$$

with $v(\mathbf{x}-\mathbf{y})=\delta\left(x_{0}-y_{0}\right) v_{L}(x-y)$,

$$
\mathcal{N}(\psi)=\sum_{s= \pm} \int d \mathbf{x} \psi_{\mathbf{x}, s}^{+} \psi_{\mathbf{x}, s}^{-}
$$

and

$$
\begin{aligned}
\nu_{C} & =2 \lambda \hat{v}_{L}(0) r_{C}:=\lambda \hat{v}_{L}(0)\left[g^{\beta, L}\left(0,0^{+}\right)-g^{\beta, L}\left(0,0^{-}\right)\right] \\
-e_{C} & :=-\lambda \hat{v}_{L}(0) r_{C}^{2}+\nu r_{C}
\end{aligned}
$$

Note that, while the presence in the interaction of the term $\nu \mathcal{N}(\psi)$ is needed, as explained above, to fix the Fermi momentum of the measure, the terms $\nu_{C} \mathcal{N}(\psi)$ and $r_{C} \int d \mathbf{x} J_{\mathbf{x}}^{C}$ and the constant $e_{C}$ have the role to correct the value of the free propagator at the discontinuity points, in the limit $M=\infty$, where this correction is important. To better explain this point, let us define the free energy at finite $M$ as $E^{M, \beta, L}=\log W(0,0)$, the Schwinger functions at finite $M$ as

$$
S_{n}^{M, \beta, L}\left(\mathbf{x}_{1}, s_{1}, \varepsilon_{1} ; \ldots ; \mathbf{x}_{n}, s_{n}, \varepsilon_{n}\right)=\left.\frac{\partial^{n}}{\partial \eta_{\mathbf{x}_{1}, s_{1}}^{-\varepsilon_{1}} \ldots \partial \eta_{\mathbf{x}_{n}, s_{n}}^{-\varepsilon_{n}}} \mathcal{W}(J, \eta)\right|_{0,0}
$$

and the response functions at finite $M$ as

$$
\Omega_{\alpha, \beta, L}^{M}(\mathbf{x}-\mathbf{y})=\left.\frac{\partial^{2}}{\partial J_{\mathbf{x}}^{\alpha} \partial J_{\mathbf{y}}^{\alpha}} \mathcal{W}(J, \eta)\right|_{0,0}
$$

and recall that one can express their perturbative expansion in terms of connected Feynmann graphs. Each Feynmann graph $G$ is defined by a set of internal points $\underline{\mathbf{y}}=\left(\mathbf{y}_{1}, \ldots, \mathbf{y}_{n}\right)$, associated with one of the three terms in (2.3), a set of external points $\underline{\mathbf{x}}=\left(\overline{\mathbf{x}}_{1}, \ldots, \mathbf{x}_{m}\right)$, associated with one of the three terms in (2.4), and a set of lines $l=\left(\mathbf{u}_{l}, \mathbf{z}_{l}\right)$, with $\mathbf{u}_{l}, \mathbf{z}_{l} \in \underline{\mathbf{x}} \cup \underline{\mathbf{y}}$, and has a value proportional to an integral of the form $\int d \underline{\mathbf{y}} \prod_{l \in G} \bar{g}^{\beta, L, M}\left(\mathbf{u}_{l}-\mathbf{z}_{l}\right)$. The same claim is true for the perturbative expansion of the Schwinger functions $S_{n}^{\beta, L}$, defined in (1.7), with the only difference that one has to substitute everywhere $\bar{g}^{\beta, L, M}$ with $g^{\beta, L}$, defined as in (1.15). Now, the possibility to study our model in terms of the functional $W(J, \eta)$ is of course related with the fact that the perturbative expansions coincide for $M \rightarrow \infty$. This would be trivial if no Feynmann graph had a tadpole, that is a line with $\mathbf{u}_{l}=\mathbf{z}_{l}$, or a line connecting two coinciding external points. In fact, one can see easily that, for any graph $G$,

$$
\lim _{N \rightarrow \infty} \int d \underline{\mathbf{y}} \prod_{l \in G} \bar{g}^{\beta, L, M}\left(\mathbf{u}_{l}-\mathbf{z}_{l}\right)=\int d \underline{\mathbf{y}} \prod_{l \in g} \bar{g}^{\beta, L}\left(\mathbf{u}_{l}-\mathbf{z}_{l}\right)
$$

with $\bar{g}^{\beta, L}(\mathbf{x})$ defined as in (1.20), hence equal to $g^{\beta, L}(\mathbf{x})$ for $\mathbf{x} \neq(0, n \beta)$; it follows that, if the graph $G$ has no tadpole and there are no coinciding external points, we can substitute everywhere $\bar{g}^{\beta, L}$ with $g^{\beta, L}$, by changing the integrand in a set of zero measure. Note that the lines connecting two external points can be present only in the graphs of the response functions and in the trivial graph connecting two $\eta$ fields with a free propagator; in any case, let us suppose, from now on, that there are no coinciding points. Hence, there is a problem only if there are tadpoles and, in such case, their contribution is a constant $\prod_{l \text { tadpole }} \bar{g}^{\beta, L}\left(0,0^{-}\right)$, which is different from $\prod_{l \text { tadpole }} g^{\beta, L}\left(0,0^{-}\right)$. 
Note now that, if we consider the graphs contributing to the Schwinger functions (those with at least two external lines), any tadpole can only be obtained by contracting the two fields based on one of the two vertices of a $\lambda$ term, while the other two fields are contracted with two other fields based on two other (possibly coinciding) vertices; hence, the presence of a tadpole implies that in the value of $G$ there is a factor of the form

$$
\bar{g}^{\beta, L}\left(\mathbf{x}_{1}-\mathbf{x}\right)\left(2 \nu_{T}\right) \bar{g}^{\beta, L}\left(\mathbf{x}-\mathbf{x}_{2}\right), \quad 2 \nu_{T}:=-\lambda \hat{v}_{L}(0)\left[g^{L, \beta}\left(0,0^{+}\right)+g^{L, \beta}\left(0,0^{-}\right)\right]
$$

where we used (1.21) and the fact that there are two ways to choose the couple of fields contracted in the tadpole. On the other hand, given a graph $G$ of this type, there is another graph $\widetilde{G}$, which differs from it only because, in place of the term $\mathcal{V}(\psi)$ which produced the tadpole, there is a vertex $\nu_{C} \mathcal{N}(\psi)$. If we sum the values of $G$ and $\widetilde{G}$, we get a number which is equal to the value of $G$, with $2 \nu_{T}+\nu_{C}=-2 \lambda \hat{v}_{L}(0) g^{L, \beta}\left(0,0^{-}\right)$in place of $2 \nu_{T}$. By iterating this argument, we see that the sum over all the graph can be rewritten as the sum over the graph obtained by putting $\nu_{C}=0$ and $\bar{g}^{\beta, L}\left(\mathbf{x}_{l}-\mathbf{y}_{l}\right)=g^{\beta, L}\left(\mathbf{x}_{l}-\mathbf{y}_{l}\right)$ everywhere.

The previous procedure is not sufficient to "correct" completely the perturbative expansion of the free energy. In fact, in this case there is a graph of first order in $\nu$, whose value is $\nu\left[g^{L, \beta}\left(0,0^{+}\right)+g^{L, \beta}\left(0,0^{-}\right)\right] / 2$, and two graphs of first order in $\lambda$, one with a $\lambda$-vertex and two tadpoles, whose value is $-\left(\lambda \hat{v}_{L}(0) / 4\right)\left[g^{L, \beta}\left(0,0^{+}\right)+g^{L, \beta}\left(0,0^{-}\right)\right]^{2}$, the other with a $\nu_{C}$ vertex and one tadpole, whose value is $\left(\nu_{C} / 2\right)\left[g^{L, \beta}\left(0,0^{+}\right)+g^{L, \beta}\left(0,0^{-}\right)\right]$. Their sum is different from the correct value $\nu g^{L, \beta}\left(0,0^{-}\right)-\lambda \hat{v}_{L}(0)\left[g^{L, \beta}\left(0,0^{-}\right)\right]^{2}$, but the difference is compensated by the constant $e_{C}$.

As concerns the functional derivatives containing at least one derivative with respect to the external fields $J_{\mathbf{x}}^{\alpha}$, the only graph which is not "corrected" by the counterterm $\nu_{C} \mathcal{N}(\psi)$, is the graph with one vertex $J$ and no $\lambda$ or $\nu$ vertex. This graph has a value different from 0 only if $\alpha=C$ and, in that case, is corrected by the term $r_{C} \int d \mathbf{x} J_{\mathbf{x}}^{C}$.

Another important remark is that, for $M$ finite, the integrand in the r.h.s. of (2.2) can be seen as a polynomial in the Grassmann variables $\hat{\psi}_{\mathbf{k}, s}^{+}$, defined as the Fourier transform of the field $\psi_{\mathbf{x}, s}^{+}$:

$$
\psi_{\mathbf{x}, s}^{+}=\frac{1}{L \beta} \sum_{\mathbf{k} \in \mathcal{D}_{L, \beta}} e^{-i \mathbf{k x}} \hat{\psi}_{\mathbf{k}, s}^{+}
$$

In fact, thanks to the ultraviolet cutoff (UV cutoff) on $k_{0}$, only a finite set of the variables $\hat{\psi}_{\mathbf{k}, s}^{+}$, those such that $\chi_{0}\left(\gamma^{-M} k_{0}\right) \neq 0$, may give a contribution to the Grassmann integral, and these variables are anticommuting. Hence, the structure of the interaction implies that the integral is a polynomial in $\lambda$ and $\nu$ and that the functions $S_{n}^{M, \beta, L}\left(\mathbf{x}_{1}, s_{1}, \varepsilon_{1} ; \ldots ; \mathbf{x}_{n}, s_{n}, \varepsilon_{n}\right)$ are analytic in $\lambda$ and $\nu$ at least in a small set around $\lambda=\nu=0$.

We can now prove that the Grassmann integral (2.2) can be used to compute the thermodynamical properties of the model with Hamiltonian (1.1). This follows from the following proposition.

Proposition 2.1 Assume that, for any finite $\beta$ and $L$, there is a function $\nu^{\beta, L}(\lambda)$ such that $\nu^{\beta, L}(0)=0$ and both $\nu^{\beta, L}(\lambda)$ and the Schwinger functions at finite $M S_{n}^{M, \beta, L}$, see (2.9), with $\nu=\nu^{\beta, L}(\lambda)$, see (2.8), are analytic and bounded in

$$
D=\left\{\lambda,|\lambda|<c \varepsilon_{0} \max \left\{(\log \beta)^{-1},(\log L)^{-1}\right\}\right\} \bigcup\left\{|\lambda|<\varepsilon_{0},|\arg \lambda|<\frac{\pi}{2}+\delta\right\}
$$

with $c, \varepsilon_{0}>0,0<\delta<\pi / 2$ independent of $\beta$ and $L$, and that they are uniformly convergent as $M \rightarrow \infty$. Then, if $\lambda \in D$,

$$
S_{n}^{\beta, L}\left(\mathbf{x}_{1}, s_{1}, \varepsilon_{1} ; \ldots ; \mathbf{x}_{n}, s_{n}, \varepsilon_{n}\right)=\lim _{M \rightarrow \infty} S_{n}^{M, \beta, L}\left(\mathbf{x}_{1}, s_{1}, \varepsilon_{1} ; \ldots ; \mathbf{x}_{n}, s_{n}, \varepsilon_{n}\right)
$$


where $S_{n}^{\beta, L}$ is defined as in (1.7), with $H$ given by (1.1) with $\bar{\mu}=\mu+\nu^{\beta, L}(\lambda)$.

$A$ similar statement is true for the thermal ground state energy and the response functions.

Proof - The main point, strictly related with the fact that we are treating a fermion problem, is that, for $L$ and $\beta$ finite, $S_{n}^{\beta, L}$ is the ratio of the traces of two matrices whose coefficients are entire functions of $\lambda$ and $\nu$, hence it is the ratio of two entire functions of $\lambda$ and $\nu$. On the other hand, the hypotheses on $\nu^{\beta, L}(\lambda)$ and $S_{n}^{M, \beta, L}$ and the Weierstrass theorem imply that $\nu^{\beta, L}(\lambda)$ and $\lim _{M \rightarrow \infty} S_{n}^{M, \beta, L}$ are analytic in $D$. It follows, in particular, that $S_{n}^{\beta, L}$, calculated with $\nu=\nu^{\beta, L}(\lambda)$, is the ratio of two functions analytic in $D$; hence, it may have a singularity in a point $\lambda_{0} \in D$ only if $\operatorname{Tr}\left[\mathrm{e}^{-\beta \mathrm{H}}\right]$ vanishes there, which certainly does not happen in a neighborhood of $\lambda=0$ small enough (how small possibly depending on $L, \beta$ ), since $\nu(\lambda)$ is of order $\lambda$. Moreover, also the r.h.s. of (2.13) is analytic in a small neighborhood of $\lambda=0$ and, as we have explained above, its power expansion in $\lambda$ and $\nu$, hence also its power expansion in $\lambda$ for $\nu=\nu^{\beta, L}(\lambda)$ coincide with that of $S_{n}^{\beta, L}$; hence, the two functions coincide in a disk $\widetilde{D}_{L, \beta}$ with center in $\lambda=0$ and radius $\varepsilon_{\beta, L}$ possibly vanishing as $\beta, L \rightarrow \infty$. However, $S_{n}^{\beta, L}$, being the ratio of two functions analytic in $D$, may have only isolated poles in $D \backslash \widetilde{D}_{L, \beta}$; hence, if $E$ is the set of poles, $S_{n}^{\beta, L}$ is analytic in $D \backslash E$ and necessarily coincide with the r.h.s. of (2.13) in this set, since the two functions coincide in $\widetilde{D}_{L, \beta} \subset D \backslash E$. It follows that, if $E$ were not empty, $S_{n}^{\beta, L}$ would be unbounded in $D \backslash E$, while this is not of course true for the other function.

A similar argument can be used for the response functions and the thermal ground state energy.

The RG analysis will allow us to prove that the analyticity domain of the r.h.s. of (2.13) is indeed of the form $D$ and this allows us to extend this result to all the physical quantities studied in this paper and to prove all results described before.

The proof of Theorem 1.1 is done in two steps; first we write $\bar{\mu}=\mu+\nu, \mu=\cos p_{F, L}$ and we show that it is possible to choose $\nu(\mu, \lambda)$ so that the expansions are convergent in the zero temperature and infinite volume limit, if $\lambda \in D$; the second step is to prove that $|\partial \nu(\mu, \lambda) / \partial \mu| \leq C \varepsilon_{0}$ in $D$, so that, if $C \varepsilon_{0} \leq 1 / 2$, the equation (2.1) can be uniquely solved with respect to $p_{F}$ and the solution is of the form $p_{F}=\arccos (\bar{\mu})+O(\lambda)$ (with $p_{F}$ real for $\lambda$ real positive), that is the interacting Fermi momentum is a well defined function of the parameters in the Hamiltonian, as expected.

\subsection{The ultraviolet integration}

In the following, to simplify the notation, we shall in general drop the superscripts $M, \beta$ and $L$. Moreover, we shall denote $\mathbb{T}$ the one dimensional torus $[0,2 \pi],\left\|k-k^{\prime}\right\|_{\mathbb{T}}$ the usual distance between $k$ and $k^{\prime}$ in $\mathbb{T}$ and $\|k\|_{\mathbb{T}}=\|k-0\|_{\mathbb{T}}$. Analogously $\|\mathbf{x}-\mathbf{y}\|$ will denote the distance on the the space-time $\mathcal{C} \times[-\beta, \beta]$, with periodic boundary conditions.

We introduce a positive function $\chi\left(\mathbf{k}^{\prime}\right) \in C^{\infty}(\mathbb{T} \times \mathbb{R}), \mathbf{k}^{\prime}=\left(k^{\prime}, k_{0}\right)$, such that $\chi\left(\mathbf{k}^{\prime}\right)=\chi\left(-\mathbf{k}^{\prime}\right)$ and $\chi\left(\mathbf{k}^{\prime}\right)=1$, if $\left|\mathbf{k}^{\prime}\right|<t_{0}=a_{0} v_{F} / \gamma$, and $\chi\left(\mathbf{k}^{\prime}\right)=0$ if $\left|\mathbf{k}^{\prime}\right|>a_{0} v_{F}$, where $v_{F}=\sin p_{F}$, $a_{0}=\min \left\{\frac{p_{F}}{2}, \frac{\pi-p_{F}}{2}\right\}$ and $\left|\mathbf{k}^{\prime}\right|=\sqrt{k_{0}^{2}+v_{F}^{2}\left\|k^{\prime}\right\|_{\mathbb{T}}^{2}}$. The above definition is such that the supports of $\chi\left(k-p_{F}, k_{0}\right)$ and $\chi\left(k+p_{F}, k_{0}\right)$ are disjoint and the $C^{\infty}$ function on $\mathbb{T} \times R$

$$
\hat{f}_{u . v .}(\mathbf{k}):=1-\chi\left(k-p_{F}, k_{0}\right)-\chi\left(k+p_{F}, k_{0}\right)
$$

is equal to 0 , if $v_{F}^{2}\left\|\left[|k|-p_{F}\right]\right\|_{\mathbb{T}}^{2}+k_{0}^{2}<t_{0}^{2}$. We want to apply this identity with $k \in \mathcal{D}_{L}$; hence $k^{\prime}=k \pm p_{F} \in \mathcal{D}_{L}^{\prime}=\frac{2 \pi}{L}\left(\mathcal{C}+\frac{1}{2}\right)$, since $p_{F}=\frac{2 \pi}{L}\left(n_{F}+\frac{1}{2}\right)$. It follows that, if $\mathcal{D}_{L, \beta}^{\prime}=\mathcal{D}_{L}^{\prime} \times \mathcal{D}_{\beta}$, we can write the fermion propagator in the following way:

$$
g(\mathbf{x}-\mathbf{y})=g^{(u . v)}(\mathbf{x}-\mathbf{y})+\sum_{\omega= \pm} e^{-i \omega p_{F}(x-y)} g_{\omega}^{(i . r .)}(\mathbf{x}-\mathbf{y})
$$




$$
\begin{gathered}
g^{(u . v .)}(\mathbf{x}-\mathbf{y})=\frac{1}{\beta L} \sum_{\mathbf{k} \in \mathcal{D}_{L, \beta}} e^{-i \mathbf{k}(\mathbf{x}-\mathbf{y})} \frac{\hat{f}_{u . v .}(\mathbf{k}) \chi_{0}\left(\gamma^{-M} k_{0}\right)}{-i k_{0}+\left(\cos p_{F}-\cos k\right)} \\
g_{\omega}^{(i . r .)}(\mathbf{x}-\mathbf{y})=\frac{1}{\beta L} \sum_{\mathbf{k}^{\prime} \in \mathcal{D}_{L, \beta}^{\prime}} e^{-i \mathbf{k}^{\prime}(\mathbf{x}-\mathbf{y})} \frac{\chi\left(k^{\prime}, k_{0}\right)}{-i k_{0}+E_{\omega}\left(k^{\prime}\right)} \\
E_{\omega}\left(k^{\prime}\right)=\omega v_{F} \sin k^{\prime}+\cos p_{F}\left(1-\cos k^{\prime}\right)
\end{gathered}
$$

The properties of Grassmann integration imply the following identity for the functional integral in the r.h.s. of (2.2):

$$
\begin{aligned}
e^{\mathcal{W}(J, \eta)} & =e^{-L \beta e_{C}+r_{C} \int d \mathbf{x} J_{\mathbf{x}}^{C}} \int P\left(d \psi^{(i . r .)}\right) \int P\left(d \psi^{(u . v .)}\right) e^{-\mathcal{V}^{(M)}(\psi)+\mathcal{B}^{(M)}(\psi, J, \eta)}= \\
& =e^{-L \beta E_{0}+\mathcal{S}_{0}(J, \eta)} \int P\left(d \psi^{(i . r .)}\right) e^{-\mathcal{V}^{(0)}\left(\psi^{(i . r .)}\right)+\mathcal{B}^{(0)}\left(\psi^{(i . r .)}, J, \eta\right)}
\end{aligned}
$$

where $\psi_{\mathbf{x}, s}^{ \pm}=\sum_{\omega} e^{ \pm i \omega p_{F} x} \psi_{\mathbf{x}, \omega, s}^{(i . r .)}+\psi_{\mathbf{x}, s}^{(u . v .)}$ and $P\left(d \psi^{(u . v .)}\right)$ and $P\left(d \psi^{(i . r .)}\right)$ are the Grassmann gaussian integrations with propagator $g^{(u \cdot v .)}(\mathbf{x})$ and $g^{(i . r .)}(\mathbf{x})$ respectively; moreover

$$
\begin{aligned}
& -L \beta E_{0}+\mathcal{S}_{0}(J, \eta)-\mathcal{V}^{(0)}\left(\psi^{(i . r .)}\right)+\mathcal{B}^{(0)}\left(\psi^{(i . r .)}, J, \eta\right)= \\
& =\sum_{n \geq 1} \frac{1}{n !} \mathcal{E}_{u . v .}^{T}\left(-\mathcal{V}^{(M)}\left(\psi^{(i . r .)}+\psi^{(u . v .)}\right)+\mathcal{B}^{(M)}\left(\psi^{(i . r .)}+\psi^{(u . v .)}, J, \eta\right) ; n\right)
\end{aligned}
$$

where $\mathcal{V}^{(0)}(\psi)$ is fixed by the condition $\mathcal{V}^{(0)}(0)=0$ and, given a function $F(\psi)$ on the Grassmann algebra, which is a polynomial in the variables $\hat{\psi}_{\mathbf{k}, s}$ (see remark around (2.11), the truncated expectation $\mathcal{E}_{u . v .}^{T}\left[F\left(\psi^{(i . r .)}+\psi^{(u . v .)}\right)\right]$ is a polynomial in the variables $\psi_{\mathbf{k}, s}^{(i . r .)}$, defined as

$$
\mathcal{E}_{u . v .}^{T}\left[F\left(\psi^{(i . r .)}+\psi^{(u . v .)}\right)\right]=\left.\frac{\partial^{n}}{\partial \lambda^{n}} \log \int P\left(d \psi^{(u . v .)}\right) e^{\left.\lambda F\left(\psi^{(i . r .)}+\psi^{(u . v .)}\right)\right]}\right|_{\lambda=0}
$$

We will see that, if we put $\underline{\mathbf{x}}=\left(\mathbf{x}_{1}, \ldots, \mathbf{x}_{2 l}\right), \underline{\omega}=\left(\omega_{1}, \ldots, \omega_{2 l}\right), \underline{s}=\left(s_{1}, \ldots, s_{2 l}\right)$ and $\psi_{\underline{\mathbf{x}}, \underline{\omega}, \underline{s}}=$ $\prod_{i=1}^{l} \psi_{\mathbf{x}_{i}, \omega_{i}, s_{i}}^{+} \prod_{i=l+1}^{2 l} \psi_{\mathbf{x}_{i}, \omega_{i}, s_{i}}^{-}$, the effective potential $\mathcal{V}^{(0)}(\psi)$ can be represented as

$$
\mathcal{V}^{(0)}\left(\psi^{(i . r .)}\right)=\sum_{l \geq 1} \sum_{\underline{\omega}, \underline{s}} \int d \underline{\mathbf{x}} W_{\underline{s}, 2 l}^{(0)}(\underline{\mathbf{x}}) e^{i p_{F} \sum_{i=1}^{2 l} \varepsilon_{i} \omega_{i} x_{i}} \psi_{\underline{\underline{\mathbf{x}}, \underline{\underline{\omega}}, \underline{s}}}^{(i . r)}
$$

where $W_{\underline{s}, 2 l}^{(0)}(\underline{\mathbf{x}})=\sum_{\zeta \in A} W_{\zeta}(\underline{\mathbf{x}})$, with $A$ a finite set, and $W_{\zeta}(\underline{\mathbf{x}})=F_{\zeta}(\underline{\mathbf{x}}) \prod_{(i, j) \in L_{\zeta}} \delta\left(\mathbf{x}_{i}-\mathbf{x}_{j}\right)$, where $F_{\zeta}(\underline{\mathbf{x}})$ is a smooth function and $L_{\zeta}$ is a subset of the couples $(i, j)$. In the following we shall use the notation

$$
\int d \underline{\mathbf{x}}\left|W_{\underline{s}, 2 l}^{(0)}(\underline{\mathbf{x}})\right|:=\sum_{\zeta \in A} \int d \underline{\mathbf{x}}\left|F_{\zeta}(\underline{\mathbf{x}})\right| \prod_{(i, j) \in L_{\zeta}} \delta\left(\mathbf{x}_{i}-\mathbf{x}_{j}\right)
$$

A similar representation can be written for the functional $\mathcal{B}^{(0)}\left(\psi^{(i . r .)}, J, \eta\right)$ (containing all terms which are of order greater than 0 both in the external fields $J, \eta$ and in $\psi)$, for $\mathcal{S}^{(0)}(J, \eta)$ (containing only the terms without $\psi$ external fields), and $L \beta E_{0}$ (containing the terms without external fields). In all cases, the corresponding kernels are called $W_{\underline{\alpha}, \underline{\varepsilon}, \underline{s}, m_{\psi}, m_{J}, m_{\eta}}^{(0)}(\underline{\mathbf{x}})$, with $m_{\psi}, m_{J}, m_{\eta}$ the number of $\psi$ fields, $J$ fields, $\eta$ fields, respectively, $\underline{\mathbf{x}}=\left(\mathbf{x}_{1}, \ldots, \mathbf{x}_{m_{\psi}+m_{J}+m_{\eta}}\right)$, $\underline{\alpha}=\left(\alpha_{1}, \ldots, \alpha_{m_{J}}\right)$ (the $\alpha$ indices of the $J^{\alpha}$ fields), $\underline{\varepsilon}=\left(\varepsilon_{1}, \ldots, \varepsilon_{m_{\psi}+m_{\eta}}\right)$ (the $\varepsilon$ indices of the $\psi^{\varepsilon}$ and $\eta^{\varepsilon}$ fields in a fixed arbitrary order); note that $m_{\psi}+m_{\eta}$ has to be even, hence we shall also define $m_{\psi}+m_{\eta}=2 l$. We shall also use the notation $W_{\underline{\alpha}, \underline{\varepsilon}, \underline{s}, 2 l, 0,0}^{(0)}(\underline{\mathbf{x}})=W_{\underline{s}, 2 l}^{(0)}(\underline{\mathbf{x}})$. Moreover we 
shall define the Fourier transform $\hat{W}_{\underline{\alpha}, \underline{\varepsilon}, \underline{s}, m_{\psi}, m_{J}, m_{\eta}}^{(0)}(\underline{\mathbf{k}}), \underline{\mathbf{k}}=\left(\mathbf{k}_{1}, \ldots, \mathbf{k}_{m_{\psi}+m_{J}+m_{\eta}-1}\right)$, so that, if $m^{*}=m_{\psi}+m_{J}+m_{\eta}$

$$
W_{\underline{\alpha}, \underline{\varepsilon}, \underline{s}, m_{\psi}, m_{J}, m_{\eta}}^{(0)}=\frac{1}{(L \beta)^{\bar{m}-1}} \sum_{\underline{\mathbf{k}}} e^{i \sum_{j=1}^{m^{*}-1} \varepsilon_{j} \mathbf{k}_{j}\left(\mathbf{x}_{j}-\mathbf{x}_{m^{*}}\right)} \hat{W}_{\underline{\alpha}, \underline{\varepsilon}, \underline{s}, m_{\psi}, m_{J}, m_{\eta}}^{(0)}(\underline{\mathbf{k}})
$$

where, if $m_{J}>0, \varepsilon_{j}=+1$ for the indices corresponding to the $J$ fields.

Lemma 2.2 The constant $E_{0}$ and the kernels $W_{\underline{\alpha}, \varepsilon, s, m_{\psi}, m_{J}, m_{\eta}}^{(0)}$ are given by power series in $\lambda$ and $\nu$, convergent for $|\lambda|,|\nu| \leq \varepsilon_{0}$, for $\varepsilon_{0}$ small enough and independent of $\beta, L, M$. They satisfy the following bounds:

$$
\left|E_{0}\right| \leq C \varepsilon_{0}, \quad \int d \underline{\mathbf{x}}\left|W_{\underline{\alpha}, \underline{\varepsilon}, \underline{s}, m_{\psi}, m_{J}, m_{\eta}}^{(0)}(\underline{\mathbf{x}})\right| \leq \beta L C^{l+m} \varepsilon_{0}^{k_{l, m}},
$$

for some constant $C>0$ and $k_{l, m}=\max \{1, l-1\}$, if $m_{J}+m_{\eta}=0$, otherwise $k_{l, m}=\max \{0, l-1\}$.

Moreover, $\lim _{M \rightarrow \infty} E_{0}$ and $\lim _{M \rightarrow \infty} \hat{W}_{\underline{\alpha}, \underline{\varepsilon}, \underline{s}, m_{\psi}, m_{J}, m_{\eta}}^{(0)}(\underline{\mathbf{k}})$ do exist and are reached uniformly in $M$, so that, in particular, the limiting functions are analytic in the same domain.

The proof of Lemma 2.2 is quite standard, but we present it here with some details, as this will allow us to introduce in a simple case a number of techniques and concepts we will use throughout the paper. Note that the proof could be generalized without any problem to the multi-dimensional Hubbard model.

\subsection{Proof of Lemma 2.2}

We start writing

$$
g^{(u . v .)}(\mathbf{x})=\sum_{h=1}^{M} g^{(h)}(\mathbf{x})
$$

where

$$
g^{(h)}(\mathbf{x})=\frac{1}{\beta L} \sum_{\mathbf{k} \in \mathcal{D}_{\beta, L}} e^{-i \mathbf{k x}} \frac{\hat{f}_{u . v .}(\mathbf{k}) H_{h}\left(k_{0}\right)}{-i k_{0}+\left(\cos p_{F}-\cos k\right)}
$$

with $H_{1}\left(k_{0}\right)=\chi_{0}\left(\gamma^{-1}\left|k_{0}\right|\right)$ and, if $h \geq 2, H_{h}\left(k_{0}\right)=\chi_{0}\left(\gamma^{-h}\left|k_{0}\right|\right)-\chi_{0}\left(\gamma^{-h+1}\left|k_{0}\right|\right)$. We shall use also the notation $g^{\left[h_{1}, h_{2}\right]}:=\sum_{h=h_{1}}^{h_{2}} g^{(h)}$.

Note that, for any integer $K \geq 0, g^{(h)}(\mathbf{x})$ satisfies the bound

$$
\left|g^{(h)}(\mathbf{x})\right| \leq \frac{C_{K}}{1+\left(\gamma^{h}\left|x_{0}\right|_{\beta}+|x|_{L}\right)^{K}},
$$

where $|\cdot|_{\beta}$ is the distance on the one dimensional torus of size $\beta$ and $|\cdot|_{L}$ is the distance on the periodic lattice of size $L$. Moreover, $g^{(h)}(\mathbf{x})$ admits a Gram representation: $g^{(h)}(\mathbf{x}-\mathbf{y})=$ $\int d \mathbf{z} A_{h}^{*}(\mathbf{x}-\mathbf{z}) \cdot B_{h}(\mathbf{y}-\mathbf{z})$, with

$$
\begin{aligned}
& A_{h}(\mathbf{x})=\frac{1}{\beta L} \sum_{\mathbf{k} \in \mathcal{D}_{\beta, L}} \sqrt{f_{u . v .}(\mathbf{k}) H_{h}\left(k_{0}\right)} \frac{e^{i \mathbf{k x}}}{k_{0}^{2}+\left(\cos p_{F}-\cos k\right)^{2}} \\
& B_{h}(\mathbf{x})=\frac{1}{\beta L} \sum_{\mathbf{k} \in \mathcal{D}_{\beta, L}} \sqrt{f_{u . v .}(\mathbf{k}) H_{h}\left(k_{0}\right)} e^{i \mathbf{k x}}\left(i k_{0}+\cos p_{F}-\cos k\right)
\end{aligned}
$$

and

$$
\left\|A_{h}\right\|^{2}=\int d \mathbf{z}\left|A_{h}(\mathbf{z})\right|^{2} \leq C \gamma^{-3 h}, \quad\left\|B_{h}\right\|^{2} \leq C \gamma^{3 h},
$$


for a suitable constant $C$. Moreover

$$
\int d \mathbf{x}\left|g^{(h)}(\mathbf{x})\right| \leq C \gamma^{-h}
$$

The decomposition of the UV propagator (2.26) allows us to make the decomposition of the measure $P\left(d \psi^{(u . v .)}\right)=\prod_{h=1}^{M} P\left(d \psi^{(h)}\right)$ and the corresponding decomposition of the field $\psi_{\mathbf{x}, s}^{(u . v .)}=\sum_{h=1}^{M} \psi_{\mathbf{x}, s}^{(h)}$. Hence, we can integrate iteratively the fields $\psi^{(M)}, \psi^{(M-1)}, \ldots, \psi^{(h)}$ with $h \geq 1$ and, if we define $\psi^{(\leq 0)}=\psi^{i . r .}$ and $\psi^{(\leq h)}=\psi^{i . r .}+\sum_{j=1}^{h} \psi^{(j)}$, if $h \geq 0$, we get:

$$
e^{\mathcal{W}(J, \eta)}=e^{-L \beta E_{h}+\mathcal{S}_{h}(J, \eta)} \int P\left(d \psi^{\leq h}\right) e^{-\mathcal{V}^{(h)}\left(\psi^{(\leq h)}\right)+\mathcal{B}^{(h)}\left(\psi^{(\leq h)}, J, \eta\right)}
$$

This definition agrees with (2.2), if we put

$$
E_{M}=e_{C}, \quad \mathcal{S}_{M}(J, \eta)=r_{C} \int d \mathbf{x} J_{\mathbf{x}}^{C}
$$

Let us consider first the effective potentials on scale $h, \mathcal{V}^{(h)}\left(\psi^{(\leq h)}\right)$ and $\mathcal{B}^{(h)}\left(\psi^{(\leq h)}, J, \eta\right)$. We want to show that they can be expressed as sums of terms, each one associated to an element of a family of labeled trees; we shall call this expansion the tree expansion. The tree definition can be followed looking at Fig. 1.

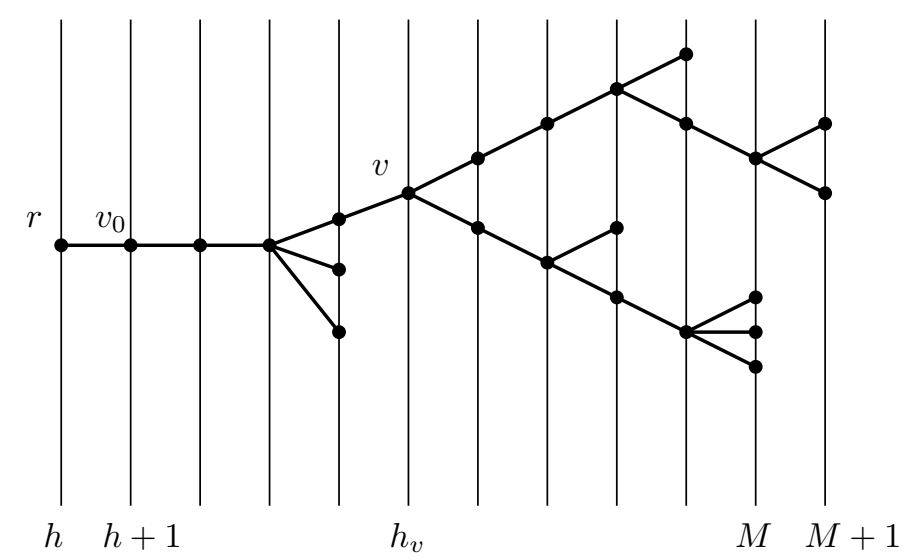

Figure 1: A tree appearing in the tree expansion of $\mathcal{V}^{(h)}$

Let us consider the family of all trees which can be constructed by joining a point $r$, the root, with an ordered set of $\bar{n} \geq 1$ points, the endpoints of the unlabeled tree, so that $r$ is not a branching point. $\bar{n}$ will be called the order of the unlabeled tree and the branching points will be called the non trivial vertices. The unlabeled trees are partially ordered from the root to the endpoints in the natural way; we shall use the symbol $<$ to denote the partial order. Two unlabeled trees are identified if they can be superposed by a suitable continuous deformation, so that the endpoints with the same index coincide. It is then easy to see that the number of unlabeled trees with $\bar{n}$ end-points is bounded by $4^{\bar{n}}$. We shall also consider the set $\mathcal{T}_{M, h, n, m}$ of the labeled trees with $n+m$ endpoints (to be called simply trees in the following); they are defined by associating some labels with the unlabeled trees, as explained in the following items. 1) We associate a label $V, J$ or $\eta$ to each endpoint, so that there are $n$ endpoints with label $V$, to be called normal endpoints, and $m=m_{J}+m_{\eta}$ endpoints, $m_{J}$ with label $J$ and $m_{\eta}$ with label $\eta$, to be called special endpoints. We shall also call $\mathcal{T}_{M, h, n, m_{J}, m_{\eta}}$ the family of trees with fixed values od $m_{J}$ and $m_{\eta}$. 
2) We associate a label $h \leq M$ with the root. Moreover, we introduce a family of vertical lines, labeled by an integer taking values in $[h, M+1]$, and we represent any tree $\tau \in \mathcal{T}_{M, h, n, m}$ so that, if $v$ is an endpoint or a non trivial vertex, it is contained in a vertical line with index $h_{v}>h$, to be called the scale of $v$, while the root $r$ is on the line with index $h$. In general, the tree will intersect the vertical lines in set of points different from the root, the endpoints and the branching points; these points will be called trivial vertices. The set of the vertices will be the union of the endpoints, of the trivial vertices and of the non trivial vertices; note that the root is not a vertex. Every vertex $v$ of a tree will be associated to its scale label $h_{v}$, defined, as above, as the label of the vertical line whom $v$ belongs to. Note that, if $v_{1}$ and $v_{2}$ are two vertices and $v_{1}<v_{2}$, then $h_{v_{1}}<h_{v_{2}}$.

3) There is only one vertex immediately following the root, which will be denoted $v_{0}$; its scale is $h+1$. If $v_{0}$ is an endpoint, the tree is called the trivial tree; this can happen only if $n+m=1$. 4) Given a vertex $v$ of $\tau \in \mathcal{T}_{M, h, n, m}$ that is not an endpoint, we can consider the subtrees of $\tau$ with root $v$, which correspond to the connected components of the restriction of $\tau$ to the vertices $w \geq v$; the number of endpoint of these subtrees will be called $n_{v}$. If a subtree with root $v$ contains only $v$ and one endpoint on scale $h_{v}+1$, it will be called a trivial subtree.

5) Given an end-point, the vertex $v$ preceding it is surely a non trivial vertex, if $n+m>1$.

Our expansion is build by associating a value to any tree $\tau \in \mathcal{T}_{M, h, n, m}$ in the following way.

First of all, given a normal endpoint $v \in \tau$ with $h_{v}=M+1$, we associate to it one of the three terms contributing to the potential $\mathcal{V}^{(M)}(\psi)$ in (2.3), that is $-\mathcal{V}\left(\psi^{(\leq M)}\right),-\nu \mathcal{N}\left(\psi^{(\leq M)}\right)$ or $-\nu_{C} \mathcal{N}\left(\psi^{(\leq M)}\right)$, while, if $h_{v} \leq M$, we associate to it one of the four terms appearing in the following expression:

$$
\begin{aligned}
-\mathcal{V}\left(\psi^{\left(<h_{v}\right)}\right) & -\nu \mathcal{N}\left(\psi^{\left(<h_{v}\right)}\right)-2 \lambda \sum_{s} \int d \mathbf{x} d \mathbf{y} v(\mathbf{x}-\mathbf{y}) g^{\left[h_{v}, M\right]}(\mathbf{x}-\mathbf{y}) \psi_{\mathbf{x}, s}^{+\left(<h_{v}\right)} \psi_{\mathbf{y}, s}^{-\left(<h_{v}\right)}+ \\
& +\left(-\nu_{C}+2 \lambda \hat{v}(0) g^{\left[h_{v}, M\right]}(\mathbf{0})\right) \sum_{s} \int d \mathbf{x} \psi_{\mathbf{x}, s}^{+\left(<h_{v}\right)} \psi_{\mathbf{x}, s}^{-\left(<h_{v}\right)}
\end{aligned}
$$

If $v$ is a special endpoint, we associate to it one of the terms contributing to the potential $\mathcal{B}^{(M)}(\psi, J, \eta)$ in (2.4), with $\psi^{\left(<h_{v}\right)}$ in place of $\psi$.

All these possible choices will be distinguished by a label $a$ in a set $A_{\tau}$, depending on $\tau$. Moreover, for any $a \in A_{\tau}$, we introduce a field label $f$ to distinguish the field variables appearing in the different terms associated to the endpoints and a source label $\alpha_{v}$ for each special endpoint; the set of field labels associated with the endpoint $v$ will be called $I_{v}$. Analogously, if $v$ is not an endpoint, we shall call $I_{v}$ the set of field labels associated with the endpoints following the vertex $v$ and $S_{v}$ the set of special endpoints following $v ; \mathbf{x}(f), \varepsilon(f), s(f)$ and $\omega(f)$ will denote the space-time point, the $\varepsilon$ index, the $s$ index and the $\omega$ index, respectively, of the Grassmann field variable with label $f$.

The previous definitions imply that, if $0 \leq h<M$, the following iterative equations are satisfied:

$$
-\mathcal{V}^{(h)}\left(\psi^{(\leq h)}\right)+\mathcal{B}^{(h)}\left(\psi^{(\leq h)}, J, \eta\right)-\beta L e_{h}+s_{h}(J, \eta)=\sum_{n=1}^{\infty} \sum_{\substack{\tau \in \mathcal{T}_{M, h, n, m} \\ a \in A_{\tau}}} \overline{\mathcal{V}}_{J}^{(h)}\left(\tau, a, \psi^{(\leq h)}\right)
$$

where, if $v_{0}$ is the first vertex of $\tau$ and $\tau_{1}, \ldots, \tau_{s}, s \geq 1$, are the subtrees with root in $v_{0}$,

$$
\overline{\mathcal{V}}_{J}^{(h)}\left(\tau, a, \psi^{(\leq h)}\right)=\frac{(-1)^{s+1}}{s !} \mathcal{E}_{h+1}^{T}\left[\overline{\mathcal{V}}_{J}^{(h+1)}\left(\tau_{1}, a_{1}, \psi^{(\leq h+1)}\right) ; \ldots ; \overline{\mathcal{V}}_{J}^{(h+1)}\left(\tau_{s}, a_{s}, \psi^{(\leq h+1)}\right)\right]
$$

where $\overline{\mathcal{V}}_{J}^{(h+1)}\left(\tau_{i}, \psi^{(\leq h+1)}\right)$ is equal to $\overline{\mathcal{V}}_{J}^{(h+1)}\left(\tau_{i}, \psi^{(\leq h+1)}\right)$ if the subtree $\tau_{i}$ contains more than one end-point, otherwise it is given by one of the terms contributing to the potentials in (2.3) 
or (2.4), if $h_{v}=N+1$, or one of the addends in (2.34), if $h_{v} \leq M$, the choice depending on the label $a$.

The identity (2.35) implies, in particular, that the constant $E_{h}$ and the functional $\mathcal{S}_{h}(J)$ defined in (2.32) are given by

$$
E_{h}=\sum_{j=h}^{M} e_{j}, \quad \mathcal{S}_{h}(J, \eta)=\sum_{j=h}^{M} s_{h}(J, \eta)
$$

with $E_{M}=e_{M}$ and $\mathcal{S}_{M}(J, \eta)=s_{M}(J, \eta)$ given by (2.33).

Note that

$$
\frac{1}{L \beta} \int d \mathbf{x} d \mathbf{y}\left|v(\mathbf{x}-\mathbf{y}) g^{[h, M]}(\mathbf{x}-\mathbf{y})\right| \leq C \gamma^{-h}
$$

and

$$
\left|-\nu_{C}+2 \lambda \hat{v}(0) g^{[h, M]}(\mathbf{0})\right| \leq C|\lambda|
$$

with a constant $C$ independent of $M$ and $h$.

The above definitions imply, in particular, that, if $n+m>1$ and $v$ is not an endpoint, then $n(v)>1$, with $n(v)$ denoting the number of endpoints following $v$ on $\tau$; in fact the vertex preceding an end-point is necessarily non trivial, if $n+m>1$.

Using its inductive definition, the right hand side of (2.35) can be further expanded, and in order to describe the resulting expansion we need some more definitions.

We associate with any vertex $v$ of the tree a subset $P_{v}$ of $I_{v}$, the external fields of $v$, and the set $\mathbf{x}_{v}$ of all space-time points associated with one of the end-points following $v$; moreover, we shall denote $\mathbf{x}_{v}^{J} \subset \mathbf{x}_{v}$ and $\mathbf{x}_{v}^{\eta} \subset \mathbf{x}_{v}$ the set of all space time points associated with the special endpoints following $v$ of type $J$ and $\eta$, respectively. The subsets $P_{v}$ must satisfy various constraints. First of all, $\left|P_{v}\right| \geq 2$, if $v>v_{0}$; moreover, if $v$ is not an endpoint and $v_{1}, \ldots, v_{s_{v}}$ are the $s_{v} \geq 1$ vertices immediately following it, then $P_{v} \subseteq \cup_{i} P_{v_{i}}$; if $v$ is an endpoint, $P_{v}=I_{v}$. If $v$ is not an endpoint, we shall denote by $Q_{v_{i}}$ the intersection of $P_{v}$ and $P_{v_{i}}$; this definition implies that $P_{v}=\cup_{i} Q_{v_{i}}$. The union $\mathcal{I}_{v}$ of the subsets $P_{v_{i}} \backslash Q_{v_{i}}$ is, by definition, the set of the internal fields of $v$, and is non empty if $s_{v}>1$. Given $\tau \in \mathcal{T}_{M, h, n, m}$, there are many possible choices of the subsets $P_{v}, v \in \tau$, compatible with all the constraints. We shall denote $\mathcal{P}_{\tau}$ the family of all these choices and $\mathbf{P}$ the elements of $\mathcal{P}_{\tau}$.

With these definitions, we can rewrite $\overline{\mathcal{V}}_{J}^{(h)}\left(\tau, a, \Psi^{(\leq h)}\right)$ in the r.h.s. of (2.35) as:

$$
\begin{aligned}
& \overline{\mathcal{V}}_{J}^{(h)}\left(\tau, a, \Psi^{(\leq h)}\right)=\sum_{\mathbf{P} \in \mathcal{P}_{\tau}} \overline{\mathcal{V}}_{J}^{(h)}(\tau, a, \mathbf{P}) \\
& \overline{\mathcal{V}}_{J}^{(h)}(\tau, a, \mathbf{P})=\int d \mathbf{x}_{v_{0}} \widetilde{\Psi}^{(\leq h)}\left(P_{v_{0}}\right) \widetilde{J}\left(S_{v_{0}}\right) \widetilde{\eta}\left(H_{v_{0}}\right) K_{\tau, \mathbf{P}}^{(h+1)}\left(\mathbf{x}_{v_{0}}\right),
\end{aligned}
$$

where $S_{v}$ and $H_{v}$ denote the set of endpoints of type $J$ and $\eta$, respectively, following $v$ and

$$
\widetilde{\psi}^{(\leq h)}\left(P_{v}\right)=\prod_{f \in P_{v}} \psi_{\mathbf{x}(f), s(f)}^{(\leq h) \varepsilon(f)}, \quad \widetilde{J}\left(S_{v}\right)=\prod_{v \in S_{v}} J_{\mathbf{x}_{v}}^{\alpha_{v}}, \quad \widetilde{\eta}\left(H_{v}\right)=\prod_{v \in H_{v}} \eta_{\mathbf{x}_{v}}^{\varepsilon_{v}}
$$

and $K_{\tau, \mathbf{P}}^{(h+1)}\left(\mathbf{x}_{v_{0}}\right)$ is defined inductively by the equation, valid for any $v \in \tau$ which is not an endpoint,

$$
K_{\tau, \mathbf{P}}^{\left(h_{v}\right)}\left(\mathbf{x}_{v}\right)=\frac{1}{s_{v} !} \prod_{i=1}^{s_{v}}\left[K_{v_{i}}^{\left(h_{v}+1\right)}\left(\mathbf{x}_{v_{i}}\right)\right] \mathcal{E}_{h_{v}}^{T}\left[\widetilde{\psi}^{\left(h_{v}\right)}\left(P_{v_{1}} \backslash Q_{v_{1}}\right), \ldots, \widetilde{\psi}^{\left(h_{v}\right)}\left(P_{v_{s_{v}}} \backslash Q_{v_{s_{v}}}\right)\right]
$$

where $\widetilde{\Psi}^{\left(h_{v}\right)}\left(P_{v_{i}} \backslash Q_{v_{i}}\right)$ has a definition similar to (2.41). Moreover, if $v_{i}$ is an endpoint, $K_{v_{i}}^{\left(h_{v}+1\right)}\left(\mathbf{x}_{v_{i}}\right)$ is equal to the kernel of one of the terms contributing to the potential in (2.3) 
or (2.4), if $h_{v_{i}}=N+1$, or one of the four terms in (2.34), if $h_{v_{i}} \leq N$; if $v_{i}$ is not an endpoint, $K_{v_{i}}^{\left(h_{v}+1\right)}=K_{\tau_{i}, \mathbf{P}_{i}}^{\left(h_{h}+1\right)}$, where $\mathbf{P}_{i}=\left\{P_{w}, w \in \tau_{i}\right\}$.

In order to get the final form of our expansion, we need a convenient representation for the truncated expectation in the r.h.s. of (2.42). Let us put $s=s_{v}, P_{i} \equiv P_{v_{i}} \backslash Q_{v_{i}}$; moreover we order in an arbitrary way the sets $P_{i}^{ \pm} \equiv\left\{f \in P_{i}, \varepsilon(f)= \pm\right\}$, we call $f_{i j}^{ \pm}$their elements and we define $\mathbf{x}^{(i)}=\cup_{f \in P_{i}^{-}} \mathbf{x}(f), \mathbf{y}^{(i)}=\cup_{f \in P_{i}^{+}} \mathbf{y}(f), \mathbf{x}_{i j}=\mathbf{x}\left(f_{i j}^{-}\right), \mathbf{y}_{i j}=\mathbf{x}\left(f_{i j}^{+}\right)$. Note that $\sum_{i=1}^{s}\left|P_{i}^{-}\right|=\sum_{i=1}^{s}\left|P_{i}^{+}\right| \equiv k$, otherwise the truncated expectation vanishes. A couple $l \equiv$ $\left(f_{i j}^{-}, f_{i^{\prime} j^{\prime}}^{+}\right) \equiv\left(f_{l}^{-}, f_{l}^{+}\right)$will be called a line joining the fields with labels $f_{i j}^{-}, f_{i^{\prime} j^{\prime}}^{+}$. Then, by using the Brydges-Battle-Federbush formula (see [23, 24]), we get, if $s>1$,

$$
\mathcal{E}_{h}^{T}\left(\widetilde{\psi}^{(h)}\left(P_{1}\right), \ldots, \widetilde{\psi}^{(h)}\left(P_{s}\right)\right)=\sum_{T} \prod_{l \in T}\left[g^{(h)}\left(\mathbf{x}_{l}-\mathbf{y}_{l}\right)\right] \int d P_{T}(\mathbf{t}) \operatorname{det} G^{h, T}(\mathbf{t}),
$$

where $T$ is a set of lines forming an anchored tree graph among the clusters of points $\mathbf{x}^{(i)} \cup \mathbf{y}^{(i)}$, that is $T$ is a set of lines, which becomes a tree graph if one identifies all the points in the same cluster. Moreover $\mathbf{t}=\left\{t_{i i^{\prime}} \in[0,1], 1 \leq i, i^{\prime} \leq s\right\}, d P_{T}(\mathbf{t})$ is a probability measure with support on a set of $\mathbf{t}$ such that $t_{i i^{\prime}}=\mathbf{u}_{i} \cdot \mathbf{u}_{i^{\prime}}$ for some family of vectors $\mathbf{u}_{i} \in \mathbb{R}^{s}$ of unit norm. Finally $G^{h, T}(\mathbf{t})$ is a $(k-s+1) \times(k-s+1)$ matrix, whose elements are given by

$$
G_{i j, i^{\prime} j^{\prime}}^{h, T}=t_{i i^{\prime}} \delta_{s_{i j}, s_{i^{\prime} j^{\prime}}} g^{(h)}\left(\mathbf{x}_{i j}-\mathbf{y}_{i^{\prime} j^{\prime}}\right)
$$

with $\left(f_{i j}^{-}, f_{i^{\prime} j^{\prime}}^{+}\right)$not belonging to $T$ and $s_{i j}, s_{i^{\prime} j^{\prime}}$ the corresponding spin variables. In the following we shall use (2.43) even for $s=1$, when $T$ is empty, by interpreting the r.h.s. as equal to 1 , if $\left|P_{1}\right|=0$, otherwise as equal to $\operatorname{det} G^{h}=\mathcal{E}_{h}^{T}\left(\widetilde{\psi}^{(h)}\left(P_{1}\right)\right)$.

The 1.h.s. of (2.35) can also be written in the form, analogous to (2.22),

$$
\begin{aligned}
& \mathcal{V}^{(h)}\left(\psi^{(\leq h)}\right)+\mathcal{B}^{(h)}\left(\psi^{(\leq h)}, J, \eta\right)+\beta L e_{h}+s_{h}(J, \eta)= \\
& \left.\sum_{m_{\psi}, m_{J}, m_{\eta} \geq 0} \sum_{\underline{\alpha}, \underline{\varepsilon}, \underline{s}} \int d \underline{\mathbf{x}} d \underline{\mathbf{y}} d \underline{\mathbf{z}} W_{\underline{\alpha}, \underline{\varepsilon}, \underline{s}, m_{\psi}, m_{J}, m_{\eta}}^{(h, M}, \underline{\mathbf{y}}, \underline{\mathbf{z}}\right) \psi_{\underline{\mathbf{x}}, \underline{s}}^{(\leq h)} J_{\underline{\alpha}, \underline{\mathbf{y}}} \eta_{\underline{\varepsilon}, \underline{\mathbf{z}}}
\end{aligned}
$$

where $\underline{\mathbf{x}}=\left(\mathbf{x}_{1}, \ldots, \mathbf{x}_{m_{\psi}}\right), \underline{\mathbf{y}}=\left(\mathbf{y}_{1}, \ldots, \mathbf{y}_{m_{J}}\right), \underline{\mathbf{z}}=\left(\mathbf{z}_{1}, \ldots, \mathbf{z}_{m_{\eta}}\right)$. The kernels $W^{(h, M)}$ admit a tree expansion that can be easily obtained from the previous discussion. Note that these kernels coincide, for $h=0$, with those of Lemma 2.2. only if $m_{\psi}>0$, otherwise they are the kernels of the terms $\beta L e_{h}$ and $s_{h}(J)$, which have to be summed up over $h$ to get the corresponding kernels, see (2.37).

If $\varepsilon_{0}=\max \{|\lambda|,|\nu|\}$, by using (2.42) and (2.43), we get the bound

$$
\begin{aligned}
& \frac{1}{\beta L} \int d \underline{\mathbf{x}} d \underline{\mathbf{y}} d \underline{\mathbf{z}}\left|W_{\underline{\alpha}, \underline{\varepsilon}, \underline{s}, m_{\psi}, m_{J}, m_{\eta}}^{(h, M}(\underline{\mathbf{x}}, \underline{\mathbf{y}}, \underline{\mathbf{z}})\right| \leq \sum_{n \geq k_{l, m}}\left(C \varepsilon_{0}\right)^{n} \sum_{\substack{\tau \in \mathcal{T}_{M, h, n, m_{J}, m_{\eta}} \\
a \in A_{\tau}}} \sum_{\substack{\mathbf{P} \in \mathcal{P}_{\tau} \\
\left|P_{v_{0}}\right|=m_{\psi}}} . \\
& \cdot \sum_{T \in \mathbf{T}} \int \prod_{l \in T} d\left(\mathbf{x}_{l}-\mathbf{y}_{l}\right) \cdot\left[\prod_{v \text { not e.p. }} \frac{1}{s_{v} !} \max _{\mathbf{t}_{v}}\left|\operatorname{det} G^{h_{v}, T_{v}}\left(\mathbf{t}_{v}\right)\right| \prod_{l \in T_{v}}\left|g^{\left(h_{v}\right)}\left(\mathbf{x}_{l}-\mathbf{y}_{l}\right)\right|\right]
\end{aligned}
$$

where, given the tree $\tau, \mathbf{T}$ is the family of all tree graphs joining the space-time points associated to the endpoints, which are obtained by taking, for each non trivial vertex $v$, one of the anchored tree graph $T_{v}$ appearing in (2.43), and by adding the lines connecting the two vertices associated to non local endpoints.

A standard application of Gram-Hadamard inequality, combined with (2.30), see [24, 13, 21], implies the dimensional bound (without factorials):

$$
\left|\operatorname{det} G^{h_{v}, T_{v}}\left(\mathbf{t}_{v}\right)\right| \leq C^{\sum_{i=1}^{s_{v}}\left|P_{v_{i}}\right|-\left|P_{v}\right|-2\left(s_{v}-1\right)} .
$$


By the decay properties of $g^{(h)}(\mathbf{x})$ given by (2.31), it also follows that

$$
\prod_{v \text { not e.p. }} \frac{1}{s_{v} !} \int \prod_{l \in T_{v}} d\left(\mathbf{x}_{l}-\mathbf{y}_{l}\right)\left\|g_{\omega_{l}}^{\left(h_{v}\right)}\left(\mathbf{x}_{l}-\mathbf{y}_{l}\right)\right\| \leq C^{n+m} \prod_{v \text { not e.p. }} \frac{1}{s_{v} !} \gamma^{-h_{v}\left(s_{v}-1\right)}
$$

We can now perform the sum $\sum_{T \in \mathbf{T}}$, which erases the $1 / s_{v}$ ! up to a $C^{n}$ factor. Then, by using the identity $\sum_{v^{\prime} \geq v}\left(s_{v^{\prime}}-1\right)=n_{v}-1$ and the bound $\sum_{v \geq v_{0}}\left[\sum_{i=1}^{s_{v}}\left|P_{v_{i}}\right|-\left|P_{v}\right|-2\left(s_{v}-1\right)\right] \leq$ $4 n+2 m-2(n+m-1)$, we easily get the final bound

$$
\begin{aligned}
& \frac{1}{\beta L} \int d \underline{\mathbf{x}} d \underline{\mathbf{y}} d \underline{\mathbf{z}}\left|W_{\underline{\alpha}, \underline{\varepsilon}, \underline{s}, m_{\psi}, m_{J}, m_{\eta}}^{(h, M)}(\underline{\mathbf{x}}, \underline{\mathbf{y}}, \underline{\mathbf{z}})\right| \leq \sum_{n \geq k_{l, m}} C^{n+m} \varepsilon_{0}^{n} \sum_{\substack{\tau \in \mathcal{T}_{M, h, n, m_{J}, m_{\eta}} \\
a \in A_{\tau}}} . \\
& \cdot \sum_{\substack{\mathbf{P} \in \mathcal{P}_{\tau} \\
\left|P_{v_{0}}\right|=m_{\psi}}} \gamma^{-h(n-1)}\left[\prod_{v \text { not trivial }} \gamma^{\left.-\left(h_{v}-h_{v^{\prime}}\right)\left(n_{v}-1\right)\right]}\right.
\end{aligned}
$$

where $v^{\prime}$ is the non trivial vertex immediately preceding $v$ or $v_{0}$. This bound is suitable to control the expansion, if $n+m>1$, since $n_{v}>1$ for any non trivial vertex, see above, and there is in such case at least one non trivial vertex. If $n+m=1$, the resummation implicit in the definition (2.34) of the terms associated to the endpoints implies that the allowed trees have only one endpoint of scale $h+1$, hence there is no problem.

Note that $\sum_{T \in \mathbf{T}}$ can be bounded by $\prod_{v} s_{v} ! C^{\sum_{i=1}^{s_{v}}\left|P_{v_{i}}\right|-\left|P_{v}\right|-2\left(s_{v}-1\right)} \leq c^{n+m} \prod_{v} s_{v}$ !, see again [24, 13, 21]. In order to bound the sum over $\tau$ and $a \in A_{\tau}$, note that the number of unlabeled trees is $\leq 4^{n}$ and that, given $\tau,\left|A_{\tau}\right| \leq C^{M}$; moreover, as $n(v) \geq 2$ and, if $v>v_{0}, 2 \leq\left|P_{v}\right| \leq$ $4 n_{v}-2\left(n_{v}-1\right)$, so that $n_{v}-1 \geq\left|P_{v}\right| / 6$,

$$
\left[\prod_{v \text { not trivial }} \gamma^{-\left(h_{v}-h_{v^{\prime}}\right)(n(v)-1)}\right] \leq\left[\prod_{v \text { not trivial }} \gamma^{-\frac{2}{5}\left(h_{v}-h_{v^{\prime}}\right)}\right]\left[\prod_{v \text { not e.p. }} \gamma^{-\frac{\left|P_{v}\right|}{10}}\right]
$$

The factor $\gamma^{-\frac{2}{5}\left(h_{v}-h_{v^{\prime}}\right)}$ can be used to bound the sum over the scale labels of the tree; moreover, see [13],

$$
\sum_{\mathbf{P} \in \mathcal{P}_{\tau}} \gamma^{-\frac{\left|P_{v}\right|}{10}} \leq C^{n+m}
$$

Since the constant $C$ is independent of $M, \beta, L$, the bounds above imply analyticity of the kernels in $\lambda$ and $\nu$, if $\varepsilon_{0}$ is small enough.

Finally in order to prove the uniform convergence as $M \rightarrow \infty$, we shall first consider the case $l \geq 1, m=0$ and we prove that, if $M^{\prime}>M$ and $0<\vartheta<1$, there is a constant $C_{\vartheta}$ such that

$$
\int d \underline{\mathbf{x}}\left|W_{\underline{s}, 2 l}^{\left(0, M^{\prime}\right)}(\underline{\mathbf{x}})-W_{\underline{s}, 2 l}^{(0, M)}(\underline{\mathbf{x}})\right| \leq C_{\vartheta} \varepsilon_{0}^{\max \{1, l-1\}} \gamma^{-\vartheta M}
$$

In order to prove this bound, we note that the tree expansion of $W_{\underline{s}, 2 l}^{\left(0, M^{\prime}\right)}(\underline{\mathbf{x}})$ differs from that of $W_{\underline{s}, 2 l}^{(0, M)}(\underline{\mathbf{x}})$ only for two reasons:

1) The trees contributing to $W_{\underline{s}, 2 l}^{\left(0, M^{\prime}\right)}(\underline{\mathbf{x}})$ are the same contributing to $W_{\underline{s}, 2 l}^{(0, M)}(\underline{\mathbf{x}})$ plus a set of trees with at least one endpoint of scale $h_{0}>M+1$. It is easy to see that the sum over the values associated to these trees satisfies a bound like (2.52), which differs from the overall bound (2.25) only for a factor $\gamma^{-\vartheta M}$. This factor is obtained by taking, for each tree $\tau$ of this type, an arbitrary endpoint $v_{0}$ of scale $h_{0} \geq M+1$ and by extracting from the bound (2.49) a factor $\gamma^{-\vartheta\left(h_{v}-h_{v^{\prime}}\right)}$ for each line connecting two non trivial vertices on the path which connects $v_{0}$ with the root on $\tau$. This operation changes the factors $\gamma^{-\left(h_{v}-h_{v^{\prime}}\right)(n(v)-1)}$ associated to these lines in $\gamma^{-\left(h_{v}-h_{v^{\prime}}\right)(n(v)-1-\vartheta)}$, which is still good enough for the bounds following (2.49), since $n(v) \geq 2$ and $\vartheta<1$. 
2) Note that the single scale propagator (2.27) is independent of $M$, for any $h \leq M$. Hence, the other trees contributing to $W_{\underline{s}, 2 l}^{\left(0, M^{\prime}\right)}(\underline{\mathbf{x}})$ differ from the corresponding trees contributing to $W_{\underline{s}, 2 l}^{\left(0, M^{\prime}\right)}(\underline{\mathbf{x}})$ only because, for some choices of the label $\alpha \in A_{\tau}$, the potentials associated to some endpoints, those depending on $g^{\left[h_{v}, M^{\prime}\right]}$, are substituted with $g^{\left[h_{v}, M\right]}$. For all these labels, the difference between the corresponding tree values can be written, if $n$ is the order of the tree $\tau$, as the some over at most $n$ terms, such that there is at least one endpoint whose associated potential contains $g^{\left[h_{v}, M^{\prime}\right]}-g^{\left[h_{v}, M\right]}$. On the other hand, by (2.27),

$$
\begin{gathered}
g^{\left[h_{v}, M^{\prime}\right]}-g^{\left[h_{v}, M\right]}(\mathbf{x})=\frac{1}{L} \sum_{k \in \mathcal{D}_{L}} e^{-i k x} \Delta_{M, M^{\prime}}\left(k, x_{0}\right) \\
\Delta_{M, M^{\prime}}\left(k, x_{0}\right)=\frac{1}{\beta} \sum_{k_{0} \in \mathcal{D}_{\beta}} e^{-i k_{0} x_{0}} \frac{\hat{f}_{u . v .}(\mathbf{k})\left[H_{M^{\prime}}\left(k_{0}\right)-H_{M+1}\left(k_{0}\right)\right]}{-i k_{0}+\left(\cos p_{F}-\cos k\right)}
\end{gathered}
$$

so that, by proceeding as in the proof of (1.20) in App. A, we can easily prove that $\mid g^{\left[h_{v}, M^{\prime}\right]}(\mathbf{x})-$ $g^{\left[h_{v}, M\right]}(\mathbf{x}) \mid \leq C \gamma^{-M}$. It follows that the bound (2.52) is verified also by the sum over the values associated to these trees.

The bound (2.52) implies that, for any $\underline{\mathbf{k}}=\left(\mathbf{k}_{1}, \ldots, \mathbf{k}_{2 l}\right)$, the sequence of functions $F_{M}(\lambda, \nu):=$ $\hat{W}_{\underline{s}, 2 l}^{(0, M)}(\underline{\mathbf{k}}), M \geq 0$, is a Cauchy sequence, uniformly in $\underline{\mathbf{k}}$ and in the domain $|\lambda|,|\nu| \leq \varepsilon_{0}$, where the $F_{M}(\lambda, \nu)$ are analytic. Hence, by Weierstrass theorem, the kernels $\hat{W}_{\underline{s}, 2 l}^{(0, M)}(\underline{\mathbf{k}})$ admit a limit $\hat{W}_{\underline{s}, 2 l}^{(0)}(\underline{\mathbf{k}})$ as $M \rightarrow \infty$; the limit is analytic in $|\lambda|,|\nu| \leq \varepsilon_{0}$ and its Taylor coefficients are the limits of the coefficients of $\hat{W}_{\underline{s}, 2 l}^{(0, M)}(\underline{\mathbf{k}})$.

Let us now consider the constant $E_{0}$, which can be written as in (2.37). We can write $e_{j}$ in terms of a tree expansion, which can be described exactly as before, the only difference being that the root has scale $j$ and $\left|P_{v_{0}}\right|=0$. The bound (2.49) implies that $\left|e_{j}\right| \leq C \varepsilon_{0} \gamma^{-j}$, hence $\left|E_{0}\right| \leq C \varepsilon_{0}$. The claim about $\lim _{M \rightarrow \infty} E_{0}$ is proved exactly as before.


$m_{J}+m_{\eta}>0$, if we write them as in (2.37) and use the bound (2.49).

\subsection{Infrared integration}

If $\chi\left(\mathbf{k}^{\prime}\right)$ is the function defined in $\$ 2.2$, we put, for any integer $h \leq 0$,

$$
f_{h}\left(\mathbf{k}^{\prime}\right)=\chi\left(\gamma^{-h} \mathbf{k}^{\prime}\right)-\chi\left(\gamma^{-h+1} \mathbf{k}^{\prime}\right)
$$

which has support $t_{0} \gamma^{h-1} \leq\left|\mathbf{k}^{\prime}\right| \leq t_{0} \gamma^{h+1}$ and equals 1 at $\left|\mathbf{k}^{\prime}\right|=t_{0} \gamma^{h}$; then

$$
\chi\left(\mathbf{k}^{\prime}\right)=\sum_{h=h_{L, \beta}}^{0} f_{h}\left(\mathbf{k}^{\prime}\right)
$$

where

$$
h_{L, \beta}:=\min \left\{h: t_{0} \gamma^{h+1}>\left|\mathbf{k}_{\mathrm{m}}\right|\right\} \quad \text { for } \quad \mathbf{k}_{\mathrm{m}}=(\pi / \beta, \pi / L) .
$$

For $h \leq 0$ we also define

$$
\hat{f}_{h}(\mathbf{k})=f_{h}\left(k-p_{F}, k_{0}\right)+f_{h}\left(k+p_{F}, k_{0}\right)
$$

(for $h=1$ the definition is (2.14) ). This definition implies that, if $h \leq 0$, the support of $\hat{f}_{h}(\mathbf{k})$ is the union of two disjoint sets, $A_{h}^{+}$and $A_{h}^{-}$. In $A_{h}^{+}, k$ is strictly positive and $\left\|k-p_{F}\right\|_{\mathbb{T}} \leq t_{0} \gamma^{h} \leq t_{0}$, 
while, in $A_{h}^{-}, k$ is strictly negative and $\left\|k+p_{F}\right\|_{\mathbb{T}} \leq t_{0} \gamma^{h}$. The label $h$ is called the scale or frequency label. Note that, if we redefine $\hat{f}_{1}(\mathbf{k})$ the function $\hat{f}_{u . v}(\mathbf{k})$ of (2.14), we have

$$
1=\sum_{h=h_{L, \beta}}^{1} \hat{f}_{h}(\mathbf{k})
$$

We can write the infrared propagator introduced in (2.15) in the following way

$$
g_{\omega}^{(i . r .)}(\mathbf{x}-\mathbf{y})=\sum_{h=h_{L, \beta}}^{0} g_{\omega}^{(h)}(\mathbf{x}-\mathbf{y})
$$

where

$$
g_{\omega}^{(h)}(\mathbf{x}-\mathbf{y})=\frac{1}{\beta L} \sum_{\mathbf{k}^{\prime} \in \mathcal{D}_{L, \beta}^{\prime}} e^{-i \mathbf{k}^{\prime}(\mathbf{x}-\mathbf{y})} \frac{f_{h}\left(\mathbf{k}^{\prime}\right)}{-i k_{0}+E_{\omega}\left(k^{\prime}\right)}
$$

The integration of the infrared scales $h \leq 0$ is done iteratively in the following way. Suppose that we have integrated the scales $0,-1,-2, . ., j+1$, obtaining

$$
e^{\mathcal{W}(J, \eta)}=e^{-L \beta E_{j}+\mathcal{S}_{j}(J, \eta)} \int P_{Z_{j}, C_{j}}\left(d \psi^{\leq j}\right) e^{-\mathcal{V}^{(j)}\left(\sqrt{Z_{j}} \psi^{\leq j}\right)+\mathcal{B}^{(j)}}\left(\sqrt{Z_{j}} \psi^{\leq j}, J, \eta\right)
$$

where, if we put $C_{j}\left(\mathbf{k}^{\prime}\right)^{-1}=\sum_{h=h_{L, \beta}}^{j} f_{h}\left(\mathbf{k}^{\prime}\right), P_{Z_{j}, C_{j}}$ is the Grassmann integration with propagator

$$
\frac{1}{Z_{j}} g_{\omega}^{(\leq j)}(\mathbf{x}-\mathbf{y})=\frac{1}{Z_{j}} \frac{1}{\beta L} \sum_{\mathbf{k} \in \mathcal{D}_{L, \beta}^{\prime}} e^{-i \mathbf{k}(\mathbf{x}-\mathbf{y})} \frac{C_{j}^{-1}(\mathbf{k})}{-i k_{0}+E_{\omega}\left(k^{\prime}\right)}
$$

$\mathcal{V}^{(j)}(\psi)$ is of the form

$$
\mathcal{V}^{(j)}(\psi)=\sum_{l \geq 1} \sum_{\underline{\omega}, \underline{s}} \int d \underline{\mathbf{x}} W_{\underline{\omega}, \underline{s}, 2 l}^{(j)}(\underline{\mathbf{x}}) \psi_{\underline{\mathbf{x}}, \underline{\omega}, \underline{s}}
$$

while $\mathcal{S}_{j}(J, \eta)$ and $\mathcal{B}^{(j)}\left(\psi^{\leq j}, J, \eta\right)$ contain all terms which are of order at least one in the source field $J$ and of order 0 or at least 2, respectively, in the field $\psi \leq j$. For $j=0, Z_{0}=1$ and the functional $\mathcal{V}^{(0)}, \mathcal{S}_{0}$ and $\mathcal{B}^{(0)}$ are those appearing in (2.19), with $W_{\underline{\omega}, \underline{\alpha}, \underline{\varepsilon}, \underline{s}, m_{\psi}, m_{J}, m_{\eta}}^{(0, \underline{\mathbf{x}})}=$ $W_{\underline{\alpha}, \underline{\varepsilon}, \underline{s}, m_{\psi}, m_{J}, m_{\eta}}^{(0)}(\underline{\mathbf{x}}) e^{i p_{F} \sum_{i=1}^{m_{\psi}+m_{\eta}} \varepsilon_{i} \omega_{i} x_{i}}$ (in other words, we have included in the definition of the kernel the $e^{i p_{F} \omega_{i} x_{i}}$ factors appearing in the decomposition of the infrared field associated to (2.15)). We find also convenient to write $\mathcal{V}^{(j)}(\psi)$ as

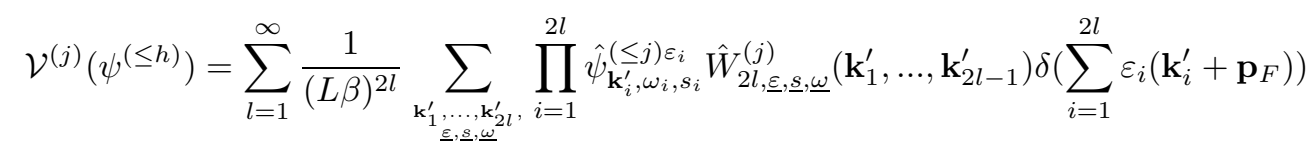

where

$$
\delta(\mathbf{k})=\delta(k) \delta\left(k_{0}\right), \quad \delta(k)=L \sum_{n \in \mathbb{Z}} \delta_{k, 2 \pi n}, \quad \delta\left(k_{0}\right)=\beta \delta_{k_{0}, 0}
$$

In order to integrate $\psi^{(j)}$ we split $\mathcal{V}^{(j)}$ as $\mathcal{L} \mathcal{V}^{(j)}+\mathcal{R} \mathcal{V}^{(j)}$, where $\mathcal{R}=1-\mathcal{L}$ and $\mathcal{L}$, the localization operator, is a linear operator on functions of the field defined in the following way by its action on the kernels $\hat{W}_{2 l, \underline{\varepsilon}, \underline{s}, \underline{\omega} \text {. }}^{(j)}$

1) If $2 l=4$, then

$$
\mathcal{L} \hat{W}_{4, \underline{\varepsilon}, \underline{s}, \underline{\omega}}^{(j)}\left(\mathbf{k}_{1}^{\prime}, \mathbf{k}_{2}^{\prime}, \mathbf{k}_{3}^{\prime}\right)=\delta_{\sum_{i=1}^{4} \varepsilon_{i} \omega_{i} p_{F}, 0} \hat{W}_{4, \underline{\varepsilon}, \underline{s}, \underline{\omega}}^{(j)}\left(\overline{\mathbf{k}}_{++}, \overline{\mathbf{k}}_{++}, \overline{\mathbf{k}}_{++}\right)
$$


where

$$
\overline{\mathbf{k}}_{\eta \eta^{\prime}}=\left(\eta \frac{\pi}{L}, \eta^{\prime} \frac{\pi}{\beta}\right)
$$

2) If $2 l=2$ and $s_{1}=s_{2}, \omega_{1}=\omega_{2}, \varepsilon_{1}+\varepsilon_{2}=0$ (otherwise $W_{2, \underline{\varepsilon}, \underline{s}, \underline{\omega},(j)}^{(j)}=0$, by spin symmetry and the compact support properties of the propagators $\left.g^{(\leq j)}\right)$,

$$
\mathcal{L} \hat{W}_{2, \underline{\varepsilon}, \underline{s}, \underline{\omega}, \underline{\underline{\omega}}}^{(j)}\left(\mathbf{k}^{\prime}\right)=\frac{1}{4} \sum_{\eta, \eta^{\prime}= \pm 1} \hat{W}_{2, \underline{\varepsilon}, \underline{s}, \underline{\omega}}^{(h)}\left(\overline{\mathbf{k}}_{\eta \eta^{\prime}}\right)\left\{1+\eta \frac{L}{\pi}\left(b_{L}+a_{L} \frac{E\left(k^{\prime}\right)}{v_{F}}\right)+\eta^{\prime} \frac{\beta}{\pi} k_{0}\right\}
$$

where

$$
a_{L} \frac{L}{\pi} \sin \frac{\pi}{L}=1, \quad \frac{\cos p_{F}}{v_{0}}\left(1-\cos \frac{\pi}{L}\right)+b_{L} \frac{L}{\pi} \sin \frac{\pi}{L}=0
$$

In order to better understand this definition, note that, if $L=\beta=\infty$,



Hence, $\mathcal{L} \hat{W}_{2, \underline{\varepsilon}, \underline{\varepsilon}, \underline{\underline{\omega}}\left(\mathbf{k}^{\prime}\right)}^{(h)}$ has to be understood as a discrete version of the Taylor expansion up to order 1. Since $a_{L}=1+O\left(L^{-2}\right)$ and $b_{L}=O\left(L^{-2}\right)$, this property would be true also if $a_{L}=1$ and $b_{L}=0$; however the choice (2.67) has the advantage to share with (2.69) another important property, that is $\mathcal{L}^{2} \hat{W}_{2, \underline{\varepsilon}, \underline{s}, \underline{\omega}}^{(h)}\left(\mathbf{k}^{\prime}\right)=\mathcal{L} \hat{W}_{2, \underline{\varepsilon}, \underline{s}, \underline{\omega}}^{(h)}\left(\mathbf{k}^{\prime}\right)$.

3) In all the other cases

$$
\mathcal{L} \hat{W}_{2 l, \underline{\varepsilon,}, \underline{s}, \underline{\omega}}^{j}\left(\mathbf{k}_{1}^{\prime}, \ldots, \mathbf{k}_{2 l-1}^{\prime}\right)=0
$$

Note that the operator $\mathcal{L}$ satisfies the relation $\mathcal{R} \mathcal{L}=0$. By the above definition we get

$$
\begin{aligned}
\mathcal{L V}^{(j)}\left(\sqrt{Z_{j}} \psi\right) & =\gamma^{j} n_{j} F_{\nu}\left(\sqrt{Z_{j}} \psi\right)+a_{j} F_{\alpha}\left(\sqrt{Z_{j}} \psi\right)+z_{j} F_{z}\left(\sqrt{Z_{j}} \psi\right) \\
& +l_{1, j} F_{1}(\psi)+l_{2, j} F_{2}\left(\sqrt{Z_{j}} \psi\right)+l_{4, j} F_{4}\left(\sqrt{Z_{j}} \psi\right)
\end{aligned}
$$

where

$$
\begin{aligned}
F_{\nu} & =\sum_{\omega, s} \int d \mathbf{x} \psi_{\mathbf{x}, \omega, s}^{+} \psi_{\mathbf{x}, \omega, s}^{-}, & F_{1} & =\frac{1}{2} \sum_{\omega, s, s^{\prime}} \int d \mathbf{x} \psi_{\mathbf{x}, \omega, s}^{+} \psi_{\mathbf{x},-\omega, s}^{-} \psi_{\mathbf{x},-\omega, s^{\prime}}^{+} \psi_{\mathbf{x}, \omega, s^{\prime}}^{-} \\
F_{\alpha} & =\sum_{\omega, s} \int d \mathbf{x} \psi_{\mathbf{x}, \omega, s}^{+} \mathcal{D} \psi_{\mathbf{x}, \omega, s}^{-}, & F_{2} & =\frac{1}{2} \sum_{\omega, s, s^{\prime}} \int d \mathbf{x} \psi_{\mathbf{x}, \omega, s}^{+} \psi_{\mathbf{x}, \omega, s}^{-} \psi_{\mathbf{x},-\omega, s^{\prime}}^{+} \psi_{\mathbf{x},-\omega, s^{\prime}}^{-} \\
F_{z} & =\sum_{\omega, s} \int d \mathbf{x} \psi_{\mathbf{x}, \omega, s}^{+} \partial_{0} \psi_{\mathbf{x}, \omega, s}^{-}, & F_{4} & =\frac{1}{2} \sum_{\omega, s} \int d \mathbf{x} \psi_{\mathbf{x}, \omega, s}^{+} \psi_{\mathbf{x}, \omega, s}^{-} \psi_{\mathbf{x}, \omega,-s}^{+} \psi_{\mathbf{x}, \omega,-s}^{-}
\end{aligned}
$$

and $\mathcal{D} \psi_{\mathbf{x}, \omega, s}=\frac{1}{L \beta} \sum_{\mathbf{k}^{\prime}} e^{i \mathbf{k}^{\prime} \mathbf{x}} E_{\omega}\left(k^{\prime}\right) \psi_{\mathbf{k}^{\prime}, \omega, s}^{+}$(see definition (2.18)). Note that

$$
l_{4,0}=2 \lambda \hat{v}(0)+O\left(\lambda^{2}\right) \quad l_{2,0}=2 \lambda \hat{v}(0)+O\left(\lambda^{2}\right) \quad l_{1,0}=2 \lambda \hat{v}\left(2 p_{F}\right)+O\left(\lambda^{2}\right)
$$

and in writing (2.71) the $S U(2)$ spin symmetry has been used. In the case of local interactions, $\hat{v}(p)=1$. We will call $F_{1}$ in (2.72) backward interaction and $F_{2}, F_{4}$ are the forward interactions; the umklapp interaction is not present in $\mathcal{L V}^{(j)}$, as well as other terms quadratic in the fields. The reason is that the condition $p_{F} \neq 0, \frac{\pi}{2}, \pi$ says that such terms are vanishing for $j$ smaller than a suitable constant (depending on $\left|p_{F}-\pi / 2\right|$ ), because they cannot satisfy the conservation of the momentum, so there is no need to localize them (more details are in 25]). 
Let us now consider $\mathcal{B}^{(j)}\left(\sqrt{Z_{j}} \psi, J, \eta\right)$. The following analysis shows that it is necessary to localize only the terms with $m_{J}=1$ and $m_{\psi}=2$. Hence we define

$$
\mathcal{L B}^{(j)}\left(\sqrt{Z_{j}} \psi, J, \eta\right)=\int d \mathbf{x} J_{\mathbf{x}}^{(\alpha)}\left[\sum_{\alpha \neq T C_{i}} \frac{Z_{j}^{(1, \alpha)}}{Z_{j}} O_{\mathbf{x}}^{(1, \alpha)}\left(\sqrt{Z_{j}} \psi\right)+\sum_{\alpha} \frac{Z_{j}^{(2, \alpha)}}{Z_{j}} O_{\mathbf{x}}^{(2, \alpha)}\left(\sqrt{Z_{j}} \psi\right)\right]
$$

where $O^{(1, \alpha)}$ are

$$
\begin{aligned}
O_{\mathbf{x}}^{(1, C)} & =\sum_{\omega, s} \psi_{\mathbf{x}, \omega, s}^{+} \psi_{\mathbf{x}, \omega, s}^{-} \\
O_{\mathbf{x}}^{\left(1, S_{i}\right)} & =\sum_{\omega, s, s^{\prime}} \psi_{\mathbf{x}, \omega, s}^{+} \sigma_{s, s^{\prime}}^{(i)} \psi_{\mathbf{x}, \omega, s^{\prime}}^{-} \\
O_{\mathbf{x}}^{(1, S C)} & =\sum_{\varepsilon, \omega, s} s e^{2 i \varepsilon \omega p_{F} x} \psi_{\mathbf{x}, \omega, s}^{\varepsilon} \psi_{\mathbf{x}, \omega,-s}^{\varepsilon}
\end{aligned}
$$

while $O^{(2, \alpha)}$ are

$$
\begin{aligned}
O_{\mathbf{x}}^{(2, C)} & =\sum_{\omega, s} e^{2 i \omega p_{F} x} \psi_{\mathbf{x}, \omega, s}^{+} \psi_{\mathbf{x},-\omega, s}^{-} \\
O_{\mathbf{x}}^{\left(2, S_{i}\right)} & =\sum_{\omega, s, s^{\prime}} e^{2 i \omega p_{F} x} \psi_{\mathbf{x}, \omega, s}^{+} \sigma_{s, s^{\prime}}^{(i)} \psi_{\mathbf{x},-\omega, s^{\prime}}^{-} \\
O_{\mathbf{x}}^{(2, S C)} & =\sum_{\varepsilon, \omega, s} s \psi_{\mathbf{x}, \omega, s}^{\varepsilon} \psi_{\mathbf{x},-\omega,-s}^{\varepsilon} \\
O_{\mathbf{x}}^{\left(2, T C_{i}\right)} & =\sum_{\varepsilon, \omega, s, s^{\prime}} e^{-i \varepsilon \omega p_{F}} \psi_{\mathbf{x}, \omega, s}^{\varepsilon} \widetilde{\sigma}_{s, s^{\prime}}^{(i)} \psi_{\mathbf{x},-\omega, s^{\prime}}^{\varepsilon}
\end{aligned}
$$

These definitions are such that the difference between $-\mathcal{V}^{(j)}+\mathcal{B}^{(j)}$ and $-\mathcal{L} \mathcal{V}^{(j)}+\mathcal{L} \mathcal{B}^{(j)}$ is made of irrelevant terms.

Note that the factor $e^{-i \varepsilon \omega p_{F}}$ in the definition of $O_{\mathbf{x}}^{\left(2, T C_{i}\right)}$ comes from the fact that the two $a^{\varepsilon}$ operators in the definition (1.9) of the triplet Cooper density are located in two different lattice sites (otherwise the density would vanish). Moreover, there is no local operator $O_{\mathbf{x}}^{\left(1, T C_{i}\right)}$ because $\sum_{s, s^{\prime}} \psi_{\mathbf{x}, \omega, s}^{\varepsilon} \widetilde{\sigma}_{s, s^{\prime}}^{(i)} \psi_{\mathbf{x}, \omega, s^{\prime}}^{\varepsilon} \equiv 0$ by anticommutation of the fermion fields.

We then renormalize the integration measure, by moving to it part of the quadratic terms of the effective potential, that is $-z_{j}(\beta L)^{-1} \sum_{\omega, s} \sum_{\mathbf{k}}\left[-i k_{0}+E_{\omega}(k)\right] \psi_{\mathbf{k}, \omega, s}^{+} \psi_{\mathbf{k}, \omega, s}^{-}$; equation (2.60) takes the form:

$$
\left.e^{\mathcal{W}(J, \eta)}=e^{-L \beta\left(E_{j}+t_{j}\right)+\mathcal{S}_{j}(J, \eta)} \int P_{\widetilde{Z}_{j-1}, C_{j}}\left(d \psi^{(\leq j)}\right) e^{-\widetilde{\mathcal{V}}^{(j)}\left(\sqrt{Z_{j}} \psi^{\leq j}\right)+\mathcal{B}^{(j)}\left(\sqrt{Z_{j}} \psi \leq j\right.}, J, \eta\right)
$$

where $\widetilde{\mathcal{V}}^{(j)}$ is the remaining part of the effective interaction, $P_{\widetilde{Z}_{j-1}, C_{j}}(d \psi \leq j)$ is the measure whose propagator is obtained by substituting in (2.61) $Z_{j}$ with

$$
\widetilde{Z}_{j-1}(\mathbf{k})=Z_{j}\left[1+z_{j} C_{j}(\mathbf{k})^{-1}\right]
$$

and $t_{j}$ is a constant coming from the normalization of the measure. It is easy to see that we can decompose the fermion field as $\psi^{(\leq j)}=\psi^{(\leq j-1)}+\psi^{(j)}$, so that

$$
P_{\widetilde{Z}_{j-1}, C_{j}}(d \psi \leq j)=P_{Z_{j-1}, C_{j-1}}\left(d \psi^{(\leq j-1)}\right) P_{Z_{j-1}, \widetilde{f}_{j}^{-1}}\left(d \psi^{(j)}\right)
$$

where $\widetilde{f}_{j}(\mathbf{k})$ (see eq. (2.90) of [21] ) has the same support and scaling properties as $f_{j}(\mathbf{k})$. Hence, if we make the field rescaling $\psi \rightarrow\left[\sqrt{Z_{j-1}} / \sqrt{Z_{j}}\right] \psi$ and call $\hat{\mathcal{V}}^{(j)}\left(\sqrt{Z_{j-1}} \psi \leq j\right)$ the new effective 
potential, we can write the integral in the r.h.s. of (2.77) in the form

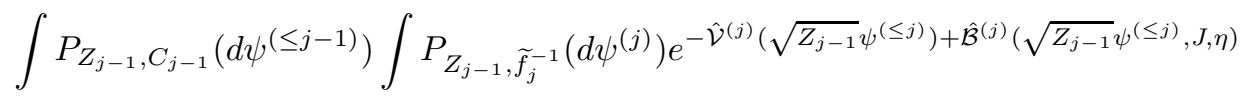

If we perform the integration over $\psi^{(j)}$ and we call

$$
E_{j-1}=E_{j}+t_{j}+\widetilde{E}_{j}, \quad \mathcal{S}_{j-1}(J, \eta)=\mathcal{S}_{j}(J, \eta)+\widetilde{\mathcal{S}}_{j}(J, \eta)
$$

the result, we finally get (2.60), with $j-1$ in place of $j$ and In order to analyze the result of this iterative procedure, we note that $\mathcal{L} \hat{\mathcal{V}}^{(j)}(\psi)$ can be written as

$$
\mathcal{L} \hat{\mathcal{V}}^{(j)}(\psi)=\gamma^{j} \nu_{j} F_{\nu}(\psi)+\delta_{j} F_{\alpha}(\psi)+g_{1, j} F_{1}(\psi)+g_{2, j} F_{2}(\psi)+g_{4, j} F_{4}(\psi)
$$

where $\nu_{j}=\left(\sqrt{Z_{j}} / \sqrt{Z_{j-1}}\right) n_{j}, \delta_{j}=\left(\sqrt{Z_{j}} / \sqrt{Z_{j-1}}\right)\left(a_{j}-z_{j}\right)$ and $g_{i, j}=\left(\sqrt{Z_{j}} / \sqrt{Z_{j-1}}\right)^{2} l_{i, j}$, $i=1,2,4$, are called the running coupling constants (r.c.c.) on scale $j$ and denoted all together by $v_{j}$. Analogously, $\mathcal{L} \hat{\mathcal{B}}^{(j)}(\psi, J)$ can be written as in (2.74), with $Z_{j}^{(i, \alpha)} / Z_{j-1}$ in place of $Z_{j}^{(i, \alpha)} / Z_{j}$.

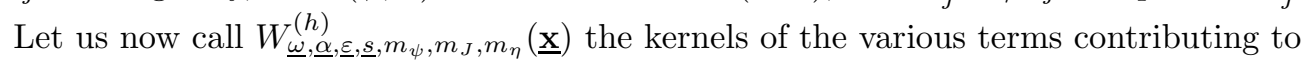
$\mathcal{B}^{(h)}\left(\sqrt{Z_{h-1}} \psi^{(\leq h-1)}, J, \eta\right)$ (in this case $m_{\psi} \neq 0$ ) or to $\widetilde{\mathcal{S}}_{h}(J, \eta)$ (in this case $m_{\psi}=0$ ). We shall prove the following Lemma, which follows from a careful dimensional analysis of the tree expansion, similar to that used in many previous papers, see for example [21].

\section{Lemma 2.3 Assume that}

$$
\max \left\{|\lambda|, \sup _{j>h}\left|v_{j}\right|\right\} \leq \varepsilon_{0}, \quad \sup _{j>h} Z_{j} / Z_{j-1} \leq e^{c_{1} \varepsilon_{0}^{2}}, \quad \sup _{\substack{j>h \\ i, \alpha}} Z_{j}^{(i, \alpha)} / Z_{j-1} \leq e^{c_{1} \varepsilon_{0}}
$$

for some $c_{1}>0$. The constant $E_{h}$ and the $\mathbf{L}^{1}$ norm of the kernels $W_{\underline{\omega}, \underline{\alpha}, \underline{\varepsilon}, \underline{s}, m_{\psi}, m_{J}, m_{\eta}}^{(h)}$ (defined as in (2.23)) are given by power series in $\left\{v_{j}\right\}_{j>h}$, convergent in the complex disc $\sup _{j>h}\left|v_{j}\right| \leq \varepsilon_{0}$, for $\varepsilon_{0}$ small enough and independent of $\beta$ and $L$; moreover, if $2 l=m_{\psi}+m_{\eta}, m=m_{J}+m_{\eta}$ and $D_{m_{\psi}, m_{J}, m_{\eta}}=-2+l+m_{J}\left(1+c_{1} \varepsilon_{0}\right)+m_{\eta}\left(1+\frac{1}{2} c_{1} \varepsilon_{0}^{2}\right)$, they satisfy the following bounds:

$$
\left|E_{h}-E_{h+1}\right| \leq C \varepsilon_{0} \gamma^{2 h}, \quad \int d \underline{\mathbf{x}}\left|W_{\underline{\omega}, \underline{\alpha}, \underline{\varepsilon}, \underline{s}, m_{\psi}, m_{J}, m_{\eta}}^{(\underline{\mathbf{x}})}\right| \leq \beta L C^{l+m} \varepsilon_{0}^{k_{l, m}} \gamma^{-h D_{m_{\psi}, m_{J}, m_{\eta}}}
$$

for some constant $C>0$ and $k_{l, m}=\max \{1, l-1\}$, if $m=0$, otherwise $k_{l, m}=\max \{0, l-1\}$.

\subsection{Proof of Lemma 2.3}

The constants $\widetilde{E}_{h}$ and the kernels $W_{\underline{\omega}, \underline{\alpha}, \underline{\varepsilon}, \underline{s}, m_{\psi}, m_{J}, m_{\eta}}^{(h)}$ can be written in terms of a tree expansion similar to that used in $\$ 2.3$, but with some important differences, which we shall describe with the help of Fig. 2.

1) The scale index now is an integer taking values in $[h, 2], h$ being the scale of the root. Moreover, there is only one vertex $v_{0}$ immediately following the root, as before, but now it can not be an endpoint. The number of endpoints is still $n+m$, but now $n_{v}$ will denote the number of normal endpoints following $v$ and we introduce three new symbols $m_{J, v}, m_{\eta, v}$ and $m_{v}=m_{J, v}+m_{\eta, v}$ to denote the number of special endpoints following $v$ of type $J$, type $\eta$ and both type, respectively.

2) With each vertex $v$ of scale $h_{v}=+1$, which is not an endpoint, we associate one of the terms contributing to $-\mathcal{V}^{(0)}\left(\psi^{(\leq 0)}\right)+\mathcal{B}^{(0)}\left(\psi^{(\leq 0)}, J, \eta\right)$, in the limit $M=\infty$, see (2.20). The endpoints of scale $h=+2$ are associated with one of the terms contributing to the potentials in (2.3) and (2.4).

3) With each endpoint $v$ of scale $h_{v} \leq 1$ we associate one of local terms that contribute to $\mathcal{L} V^{\left(h_{v}-1\right)}$, see (2.81), or $\mathcal{L} \mathcal{B}^{\left(h_{v}-1\right)}$, see (2.74), or one of the two terms linear in $\psi$ and $\eta$ appearing 


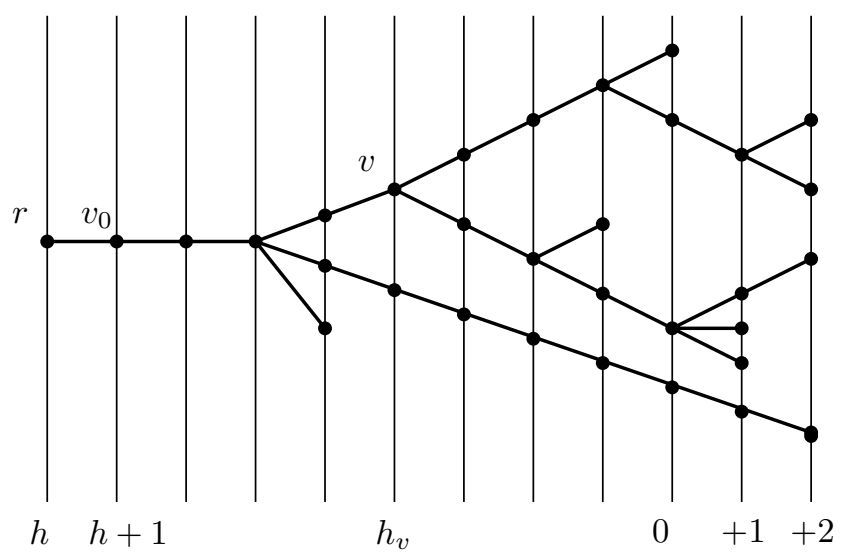

Figure 2: A renormalized tree for $\mathcal{V}^{(h)}$

in the (2.4) (recall that they are not renormalized). With each trivial or non trivial vertex $v>v_{0}$, which is not an endpoint, we associate the $\mathcal{R}=1-\mathcal{L}$ operator, acting on the corresponding kernel.

4) If $v$ is one endpoint of scale $h_{v} \leq 1$, it is still true that its scale is $h_{v^{\prime}}+1$, if $v^{\prime}$ is the non trivial vertex immediately preceding it or $v_{0}$, but this property does not apply to the endpoints of scale $h=+2$ involved in the localization procedure, that is those associated with the non local potential $\mathcal{V}(\psi)$ of (2.4); note that, in this case,the trivial vertex preceding them carry an $\mathcal{R}$ operator.

$5)$ If there is only one endpoint, the previous conditions imply that its scale must be equal to +2 or $h+2$, if $h \leq 0$. However, we need also to define the trivial tree, which is the tree with one endpoint of scale $h+1$; these trees do not belong to $\mathcal{T}_{h, n, m}$ with $n+m=1$, if $h \leq 0$, and are associated with one of the terms in the local part of $\hat{\mathcal{V}}$, see (2.81), or one of the terms in the r.h.s. of (2.74).

The previous definitions imply that the following iterative equations, similar to (2.35), are satisfied:

$$
\begin{aligned}
-\mathcal{V}^{(h)}\left(\sqrt{Z_{h}} \psi^{(\leq h)}\right) & +\mathcal{B}^{(h)}\left(\sqrt{Z_{h}} \psi^{(\leq h)}, J, \eta\right)-L \beta \widetilde{E}_{h+1}+\widetilde{\mathcal{S}}_{h}(J, \eta)= \\
& =\sum_{n=1}^{\infty} \sum_{\substack{\tau \in \mathcal{T}_{h, n, m} \\
a \in A_{\tau}}} \bar{V}_{J}^{(h)}\left(\tau, a, \sqrt{Z_{h}} \psi^{(\leq h)}\right)
\end{aligned}
$$

where, if $v_{0}$ is the first vertex of $\tau$ and $\tau_{1}, . ., \tau_{s}\left(s=s_{v_{0}}\right)$ are the subtrees of $\tau$ with root $v_{0}$, $\bar{V}_{J}^{(h)}\left(\tau, a, \sqrt{Z_{h}} \psi^{(\leq h)}\right)$ is defined inductively by the relation

$$
\begin{aligned}
& \bar{V}_{J}^{(h)}\left(\tau, a, \sqrt{Z_{h}} \psi^{(\leq h)}\right)= \\
& \frac{(-1)^{s+1}}{s !} \mathcal{E}_{h+1}^{T}\left[\bar{V}_{J}^{(h+1)}\left(\tau_{1}, a_{1}, \sqrt{Z_{h}} \psi^{(\leq h+1)}\right) ; . . . \bar{V}_{J}^{(h+1)}\left(\tau_{s}, a_{s}, \sqrt{Z_{h}} \psi^{(\leq h+1)}\right)\right]
\end{aligned}
$$

and $\bar{V}^{(h+1)}\left(\tau_{i}, a_{i}, \sqrt{Z_{h}} \psi^{(\leq h+1)}\right)$

a) is equal to $\mathcal{R} \hat{\mathcal{V}}^{(h+1)}\left(\tau_{i}, a_{i}, \sqrt{Z_{h}} \psi^{(\leq h+1)}\right)$ if the subtree $\tau_{i}$ is not trivial;

b) if $\tau_{i}$ is trivial and $h \leq-1$, it is equal to one of the terms associated with the corresponding endpoint (of scale $h+1$ ), as described in item 3 ) above, or, if $h=0$, to one of the terms in the r.h.s. of (2.3) or (2.4).

The main difference with respect to the proof of Lemma 2.2 is in the presence of the $\mathcal{R}=1-\mathcal{L}$ operators. Let us assume first that $\mathcal{R}=1$, so that, in particular, we do not perform the free 
measure renormalization; in this case we can repeat exactly the analysis leading from (2.40) to (2.49), with $\varepsilon_{0}$ having the same meaning. The only difference is just that $g_{\omega}^{(h)}(\mathbf{x})$ admits a Gram representation: $g_{\omega}^{(h)}(\mathbf{x}-\mathbf{y})=\int d \mathbf{z} A_{h}^{*}(\mathbf{x}-\mathbf{z}) \cdot B_{h}(\mathbf{y}-\mathbf{z})$, with

$$
\begin{aligned}
& A_{h}(\mathbf{x})=\frac{1}{\beta L} \sum_{\mathbf{k}^{\prime} \in \mathcal{D}_{\beta, L}^{\prime}} \sqrt{\widetilde{f}_{h}\left(\mathbf{k}^{\prime}\right)} \frac{e^{i \mathbf{k}^{\prime} \mathbf{x}}}{k_{0}^{2}+E_{\omega}\left(k^{\prime}\right)^{2}} \\
& B_{h}(\mathbf{x})=\frac{1}{\beta L} \sum_{\mathbf{k}^{\prime} \in \mathcal{D}_{\beta, L}^{\prime}} \sqrt{\widetilde{f}_{h}\left(\mathbf{k}^{\prime}\right)} e^{i \mathbf{k}^{\prime} \mathbf{x}}\left(i k_{0}+E_{\omega}\left(k^{\prime}\right)\right)
\end{aligned}
$$

and

$$
\left\|A_{h}\right\|^{2}=\int d \mathbf{z}\left|A_{h}(\mathbf{z})\right|^{2} \leq C \gamma^{-2 h}, \quad\left\|B_{h}\right\|^{2} \leq C \gamma^{4 h},
$$

for a suitable constant $C$. Therefore the Gram-Hadamard inequality implies that

$$
\left|\operatorname{det} G^{h_{v}, T_{v}}\left(\mathbf{t}_{v}\right)\right| \leq c^{\sum_{i=1}^{s_{v}}\left|P_{v_{i}}\right|-\left|P_{v}\right|-2\left(s_{v}-1\right)} \cdot \gamma^{\frac{h_{v}}{2}}\left[\sum_{i=1}^{s_{v}}\left|P_{v_{i}}\right|-\left|P_{v}\right|-2\left(s_{v}-1\right)\right] .
$$

By the decay properties of $g_{\omega}^{(h)}(\mathbf{x})$, it also follows that

$$
\prod_{v \text { not e.p. }} \frac{1}{s_{v} !} \int \prod_{l \in T_{v}} d\left(\mathbf{x}_{l}-\mathbf{y}_{l}\right)\left\|g_{\omega_{l}}^{\left(h_{v}\right)}\left(\mathbf{x}_{l}-\mathbf{y}_{l}\right)\right\| \leq C^{n+m} \prod_{v \text { not e.p. }} \frac{1}{s_{v} !} \gamma^{-h_{v}\left(s_{v}-1\right)} .
$$

Note now that $\left|I_{v}\right|=4 n_{4, v}+2 n_{2, v}+m_{\eta, v}+2 m_{J, v}$, where $n_{4, v}$ and $n_{2, v}$ are the number of normal endpoints with four and two $\psi$ fields, respectively. Hence,

$$
\begin{gathered}
\sum_{\tilde{v} \geq v}\left\{\frac{1}{2}\left(\sum_{i=1}^{s_{\tilde{v}}}\left|P_{v_{i}}\right|-\left|P_{v}\right|\right)-2\left(s_{\widetilde{v}}-1\right)\right\}=\frac{1}{2}\left(\left|I_{v}\right|-\left|P_{v}\right|\right)-2\left(n_{v}+m_{v}-1\right)= \\
=2-\frac{1}{2}\left|P_{v}\right|-n_{2, v}-m_{J, v}-\frac{3}{2} m_{\eta, v}
\end{gathered}
$$

Therefore, repeating the same steps leading from (2.41) to (2.48) we get, instead of (2.49)

$$
\sum_{n \geq k_{l, m}} C^{n+m} \varepsilon_{0}^{n} \sum_{\substack{\tau \in \mathcal{T}_{h, n, m_{J}, m_{\eta}} \\ a \in A_{\tau}}} \sum_{\substack{\mathbf{P} \in \mathcal{P}_{\tau} \\\left|\mathcal{P}_{v_{0}}\right|=m_{\psi}}} \sum_{T \in \mathbf{T}} \gamma^{-\left(D_{v_{0}}+n_{2, v_{0}}\right) h}\left[\prod_{\substack{v \\ v \text { not e.p. } \\ v>v_{0}}} \frac{1}{s_{v} !} \gamma^{-\left(D_{v}+n_{2, v}\right)}\right]
$$

where

$$
D_{v}=-2+\frac{1}{2}\left|P_{v}\right|+m_{J, v}+\frac{3}{2} m_{\eta, v}
$$

$D_{v}$ is called scaling dimension.

The fact that the scaling dimension $D_{v}$ can be negative or vanishing prevents the possibility of performing the sum over the scales, as we did in the equations leading from (2.49) to (2.51). The action of the $\mathcal{R}$ operator (2.71) has the effect that instead of (2.91) the following bound is found

$$
\begin{aligned}
& \sum_{n \geq k_{l, m}} \sum_{\substack{\tau \in \mathcal{T}_{h, n, m} \\
a \in A_{\tau}}} \sum_{\substack{\mathbf{P} \in \mathcal{P}_{\tau} \\
\left|P_{v_{0}}\right|=2 l}} \sum_{T \in \mathbf{T}} C^{n+m} \varepsilon_{0}^{n}\left[\prod_{t=1}^{m_{J}} \frac{Z_{h_{v_{t}}}^{i_{t}, \alpha_{t}}}{Z_{h_{v_{t}}-1}}\right]\left[\prod_{s=1}^{m_{\eta}} \frac{1}{\sqrt{Z_{\bar{v}_{s}}-1}}\right] . \\
& \cdot \gamma^{-D_{v_{0}} h}\left[\prod_{\substack{v \text { not e.p. } \\
v>v_{0}}}\left(\frac{Z_{h_{v}}}{Z_{h_{v}-1}}\right)^{\left|P_{v}\right| / 2} \frac{1}{s_{v} !} \gamma^{-\left[D_{v}+z\left(\left|P_{v}\right|, m_{v}\right)\right]}\right]
\end{aligned}
$$


where $\varepsilon_{0}$ is defined as in (2.82) $, v_{t}, t=1, \ldots, m_{J}$, and $\bar{v}_{s}, s=1, \ldots, m_{\eta}$, are the special endpoints of type $J$ and $\eta$, respectively; moreover, $z(2,0)=2, z(4,2)=1, z(2,1)=1$ and $z(p, m)=0$ otherwise.

The proof of this bound is by now rather standard, but does not depend at all on the details of the model, hence we address the reader to $\S 3$ of [21], where a similar bound is obtained. In any case, the change of the dimensional factors is easy to understand. First of all, $n_{2, v}$ disappears, because it is erased by the effect of the dimensional factor $\gamma^{j}$ which multiplies the r.c.c. $\nu_{j}$ in (2.81). Moreover, the presence of $z\left(\left|P_{v}\right|, m_{v}\right)$ is explained by the simple remark that the $\mathcal{R}$ operation on the kernel $W^{\left(h_{v}\right)}\left(\mathbf{k}_{1}^{\prime}, \ldots, \mathbf{k}_{2 l-1}^{\prime}\right)$, associated to the vertex $v$ of scale $h_{v}$, see (2.63), has roughly the effect of substituting it with the rest of the Taylor expansion of order $z-1$ in at least one of its variables, let us say $\mathbf{k}_{1}^{\prime}$, see (2.69). The derivative of order $z$ acting on $\mathbf{k}_{1}^{\prime}$ will produce a "bad factor" at most equal to $\gamma^{-z h_{v}}$, while the size of $\left|\mathbf{k}_{1}^{\prime}\right|$ gives a "good factor" at least equal to $\gamma^{-z h_{\widetilde{v}}}$, where $\widetilde{v}<v$ is the vertex where the external field of momentum $\mathbf{k}_{1}^{\prime}$ is contracted or $v_{0}$, if it belongs to $P_{v_{0}}$.

Note that

$$
D_{v}+z\left(\left|P_{v}\right|, m_{v}\right)>0, \quad \forall v>v_{0}
$$

except in the case $\left|P_{v}\right|=m_{\eta, v}=1$ and $m_{J, v}=0$. However, thanks to support properties of the single scale covariance in the $\mathbf{k}$ variables, this can happen only in the non trivial vertex where an endpoint of type $\eta$ is connected to the tree, otherwise the tree value vanishes. It follows immediately that this exception does not give any problem in the evaluation of the sum over the scale indices, which is out of control only if one can find an arbitrary long chain of tree vertices with non positive scale dimension.

In order to bound in (2.93) the sums over the scale labels and the set $\mathcal{P}_{\tau}$, we first use (2.82), by adding the hypothesis that $c_{1} \varepsilon_{0}, c_{1} \varepsilon_{0}^{2} \leq 1 / 16$; we get

$$
\begin{aligned}
& {\left[\prod_{t=1}^{m_{J}} \frac{Z_{h_{v_{t}}}^{i_{t}, \alpha_{t}}}{Z_{h_{v_{t}}-1}}\right]\left[\prod_{s=1}^{m_{\eta}} \frac{1}{\sqrt{Z_{h_{\bar{v}_{s}}}-1}}\right]\left[\prod_{\substack{\text { not e.p. } \\
v>v_{0}}}\left(\frac{Z_{h_{v}}}{Z_{h_{v}-1}}\right)^{\left|P_{v}\right| / 2} \gamma^{-\left[D_{v}+z\left(\left|P_{v}\right|, m_{v}\right)\right]}\right] \leq} \\
& \leq e^{m_{J} c_{1} \varepsilon_{0} h+\frac{1}{2} m_{\eta} c_{1} \varepsilon_{0}^{2}}\left[\prod_{v \text { non trivial }} \gamma^{-\frac{1}{40}\left(h_{v}-h_{v^{\prime}}\right)}\right]\left[\prod_{v \text { not e.p. }} \gamma^{-\frac{\left|P_{v}\right|}{40}}\right]
\end{aligned}
$$

Then we can continue as in the proof of Lemma 2.2

Remark 1 - An easy corollary of the above proof is that the bound for the value associated to trees with root $h$ and at least one non trivial vertex of scale $j$ can be improved by a factor $\gamma^{\vartheta(j-h)}$ with $0<\vartheta<1$. It is sufficient to notice that, thanks to (2.94), one can extract from the bound in the first line of (2.95) one factor $\gamma^{\vartheta\left(h_{v}-h_{v^{\prime}}\right)}$ for each non trivial vertex on the path $\mathcal{C}$ connecting the vertex $v^{*}$ of scale $j$ with $v_{0}$. Hence, there is $\alpha_{\vartheta}>0$ such that the bound in the second line of (2.95) can be substituted with the product of $e^{m_{J} c_{1} \varepsilon_{0} h+\frac{1}{2} m_{\eta} c_{1} \varepsilon_{0}^{2}}$ times

$$
\gamma^{\vartheta(j-h)} \prod_{\substack{\tilde{\mathcal{C}} \in \mathcal{C} \\ \text { nontrivial }}} \gamma^{-\alpha_{\vartheta}\left(h_{\widetilde{v}}-h_{\widetilde{v}^{\prime}}\right)} \prod_{\substack{v \in \mathcal{C} \\ \text { note.p. }}} \gamma^{-\alpha_{\vartheta}\left|P_{v}\right|} \prod_{\substack{v \notin \mathcal{C} \\ \text { nontrivial }}} \gamma^{-\frac{1}{40}\left(h_{v}-h_{v^{\prime}}\right)} \prod_{\substack{v \notin \mathcal{C} \\ \text { note.p. }}} \gamma^{-\frac{\left|P_{v}\right|}{40}}
$$

This important property will be called in the following the short memory property.

Remark 2 - The tree expansion has another important property, that will be used many times in the following to translate "rough" dimensional arguments into rigorous dimensional bounds. Suppose that we make a small change of one of the parameters of the model, so that the main objects involved in the tree expansion, such as the r.c.c., the ren.c.'s or the single scale propagators, are subject to a small perturbation. Then, by using the "stability" of the Gram-Hadamard 
inequality (2.47) under a small perturbation of the propagator and the short memory property, one can see, by an iterative argument, that the sum over the trees with $n$ endpoints is subject to a small variation, up to a $C^{n}$ factor in the bounds. This property, which is model independent, is explained with enough details in $\S 4.6$ of 21 in a particular case. We shall call it the stability property of the tree expansion.

\subsection{The flow of the running coupling constants}

In order to use Lemma 2.3, we must show that the assumptions (2.82) are verified for any $h>h_{L, \beta}$. Let us consider first the r.c.c. and define for them the following vector notations:

$$
\vec{v}_{h} \equiv\left(v_{1, h}, v_{2, h}, v_{4, h}, v_{\delta, h}, v_{\nu, h}\right)=\left(g_{1, h}, g_{2, h}, g_{4, h}, \delta_{h}, \nu_{h}\right) \equiv\left(\vec{g}_{h}, \delta_{h}, \nu_{h}\right) \equiv\left(\mathbf{v}_{h}, \nu_{h}\right)
$$

The r.c.c. satisfy a set of recursive equations, which can be obtained by applying the localization operator to the r.h.s. of (2.84); the result can written in the form:

$$
v_{\alpha, j-1}=A_{\alpha} v_{\alpha, j}+\hat{\beta}_{\alpha}^{(j)}\left(v_{j} ; \ldots, v_{0} ; \lambda, \nu\right)
$$

with $A_{\nu}=\gamma, A_{\alpha}=1$ for $\alpha \neq \nu$. These equations have been already analyzed in [25], where it has been proved that, if $\lambda$ is real positive and small enough, then it is possible to choose $\nu$ so that, fixed $\vartheta<1,\left|\nu_{h}\right| \leq C \lambda \gamma^{\vartheta h}, \forall h \leq 0$, and $0<g_{1, h}<\lambda(1+\bar{a} \lambda|h|)^{-1}$, for some $\bar{a}>0$, while the other r.c.c. stay bounded by $C \lambda$ and converge for $h \rightarrow-\infty$. In this paper, in order to proof Borel summability of perturbation theory, we extend the proof to complex values of $\lambda$, restricted to the set $D_{\varepsilon, \delta}$ defined in (1.28); this implies that we need an analysis a bit more precise of the flow equations (2.98).

To begin with, we put $\nu_{1} \equiv \nu$ and we suppose that the sequence $\left\{\nu_{h}\right\}_{h \leq 1}$ is made of known functions of $\lambda$, analytic in $D_{\varepsilon, \delta}$, such that

$$
\sup _{j \leq 1} \gamma^{-\vartheta j}\left|\nu_{j}\right| \leq \xi|\lambda|
$$

and study the flow equations of the other variables. The idea is that this restricted flow has properties such that, by a fixed point argument, the sequence $\left\{\nu_{h}\right\}_{h \leq 1}$, satisfying the last equation of (2.98), can be uniquely determined, for $\xi$ large enough. This point can be treated in a way similar to that used in the spinless case (see App. 5 of [26] or $\S 4.3$ of [21], where a different method is used); we shall give the main details below, see 2.8 . Hence, from now on, we shall consider the restriction of (2.98) to $\mathbf{v}_{j}$, see (2.97).

The next step is to extract from the functions $\hat{\beta}_{\alpha}^{(j)}$ the leading terms for $j \rightarrow-\infty$. Observe that the propagator $\widetilde{g}_{\omega}^{(j)}$ of the single scale measure $P_{Z_{j-1}, \widetilde{f}_{j}^{-1}}$, can be decomposed as

$$
\widetilde{g}_{\omega}^{(j)}(\mathbf{x})=\frac{1}{Z_{j}} g_{\mathrm{D}, \omega}^{(j)}(\mathbf{x})+r_{\omega}^{(j)}(\mathbf{x})
$$

where $g_{\mathrm{D}, \omega}^{(j)}$ is the Dirac propagator (with cutoff) and describes the leading asymptotic behavior

$$
g_{\mathrm{D}, \omega}^{(j)}(\mathbf{x}):=\frac{1}{\beta L} \sum_{\mathbf{k} \in \mathcal{D}_{L, \beta}} e^{-i \mathbf{k x}} \frac{\tilde{f}_{j}(\mathbf{k})}{-i k_{0}+\omega v_{F} k},
$$

while the remainder $r_{\omega}^{(j)}$ satisfies, for any $q>0$ and $0<\vartheta<1$, the bound

$$
\left|r_{\omega}^{(j)}(\mathbf{x})\right| \leq \frac{\gamma^{(1+\vartheta) j}}{Z_{j}} \frac{C_{q, \vartheta}}{1+\left(\gamma^{j}|\mathbf{x}|\right)^{q}} .
$$


Let us now call $Z_{\mathrm{D}, j}$ the values of $Z_{j}$ one would obtain by substituting $\mathcal{V}^{(0)}$ with $\mathcal{L} \mathcal{V}^{(0)}$ in (2.19) and by using for the single scale integrations the propagator $(2.100)$ with $r_{\omega}^{(i)}(\mathbf{x}) \equiv 0$ for any $i \geq j$. It can be proved by an inductive argument that, if all the r.c.c. stay of order $\lambda$,

$$
\left|\frac{Z_{j}}{Z_{j-1}}-\frac{Z_{\mathrm{D}, j}}{Z_{\mathrm{D}, j-1}}\right| \leq C \varepsilon_{j}^{2} \gamma^{\vartheta j}
$$

where

$$
\varepsilon_{j}:=\max \left\{|\lambda|, \max _{0 \geq h \geq j}\left|\vec{g}_{h}\right|, \max _{0 \geq h \geq j}\left|\delta_{h}\right|\right\}
$$

It is then convenient to decompose the functions $\hat{\beta}_{\alpha}^{(j)}$ as

$$
\hat{\beta}_{\alpha}^{(j)}\left(\vec{v}_{j} ; \ldots, \vec{v}_{0} ; \lambda, \nu\right)=\beta_{\alpha}^{(j)}\left(\mathbf{v}_{j}, \ldots, \mathbf{v}_{0}\right)+\bar{\beta}_{\alpha}^{(j)}\left(\vec{v}_{j} ; \ldots, \vec{v}_{0} ; \lambda, \nu\right)
$$

where $\beta_{\alpha}^{(j)}\left(\mathbf{v}_{j}, \ldots, \mathbf{v}_{0}\right)$ is given by the sum of all trees containing only endpoints with r.c.c. $\delta_{h}, \vec{g}_{h}$, $0 \geq h \geq j$, modified so that the propagators $g_{\omega}^{(h)}$ and the wave function renormalizations $Z_{h}$, $0 \geq h \geq j$, are replaced by $g_{\mathrm{D}, \omega}^{(h)}$ and $Z_{\mathrm{D}, h} ; \bar{\beta}_{\alpha}^{(j)}$ contains the correction terms together with the remainder of the expansion. (2.103) and (2.99) imply that there two constants $\bar{c}$ and $C$, such that

$$
\left|\bar{\beta}_{\alpha}^{(j)}\left(\vec{v}_{j} ; \ldots, \vec{v}_{0} ; \lambda\right)\right| \leq \begin{cases}C \varepsilon_{j}^{2} \gamma^{\vartheta j} & \text { if } \alpha \neq \delta \\ \left(\bar{c} \varepsilon_{0}+C \varepsilon_{j}^{2}\right) \gamma^{\vartheta j} & \text { if } \alpha=\delta\end{cases}
$$

Remark Note that the constant $C$ in (2.103) and (2.105) depends on the constant $\xi$ of (2.99). It is easy to see that, if we call $C_{1}$ the constant appearing in (2.93), then $C=C_{1} \max \left\{C_{1}, \xi\right\}$ and all bounds of this section are verified only if, say, $\max \left\{C_{1}, \xi\right\} \varepsilon_{0} \leq 1 / 2$. In the following discussion, the only constant which depends on $C$ under this smallness hypothesis, is the constant $b_{2}$ of (2.116) below. Hence, all the other constants will be independent of $\xi$, if the first condition in (2.134) is also verified.

The leading term in (2.104), that is $\beta_{\alpha}^{(j)}$, can be further decomposed as

$$
\beta_{\alpha}^{(j)}\left(\mathbf{v}_{j}, \ldots, \mathbf{v}_{0}\right)=\widetilde{\beta}_{\alpha}^{(j)}\left(\mathbf{v}_{j}\right)+r_{\alpha, j}\left(\mathbf{v}_{j}, \ldots, \mathbf{v}_{0}\right)
$$

where $\widetilde{\beta}_{\alpha}^{(j)}(\mathbf{v})=\beta_{\alpha}^{(j)}(\mathbf{v}, \ldots, \mathbf{v})$. We can write:

$$
\widetilde{\beta}_{\alpha}^{(j)}\left(\mathbf{v}_{j}\right)=\sum_{i=0,1} b_{\alpha, i}^{(j)}\left(\mathbf{v}_{j}\right)+b_{\alpha, \geq 2}^{(j)}\left(\mathbf{v}_{j}\right)
$$

where $b_{\alpha, i}^{(j)}\left(\mathbf{v}_{j}\right)$ is the contribution of order $i$ in $g_{1, j}$, wile $b_{\alpha, \geq 2}^{(j)}\left(\mathbf{v}_{j}\right)$ is the contributions of all trees with at least two endpoints of type $g_{1}$. The crucial property is the following one, called partial vanishing of the beta function, whose proof is in Appendix C

$$
\left|b_{\alpha, i}^{(j)}\left(\mathbf{v}_{j}\right)\right| \leq C \varepsilon_{j}^{2}\left[\gamma^{\vartheta j}+\gamma^{-\left(j-h_{L, \beta}\right)}\right], \quad i=0,1
$$

Now, let us extract from $\widetilde{\beta}_{\alpha}^{(j)}\left(\mathbf{v}_{j}\right)$ the second order contributions, which all belong to $b_{\alpha, \geq 2}^{(j)}\left(\mathbf{v}_{j}\right)$; we get:

$$
\widetilde{\beta}_{\alpha}^{(j)}\left(\mathbf{v}_{j}\right)=-a_{\alpha} g_{1, j}^{2}+\sum_{i=0,1} b_{\alpha, i}^{(j)}\left(\mathbf{v}_{j}\right)+\widetilde{r}_{\alpha, j}\left(\mathbf{v}_{j}\right)
$$

with $a_{1}=a_{L, \beta}^{(j)}>0, a_{2}=a_{L, \beta}^{(j)} / 2, a_{4}=a_{\delta}=0$, and, for some $b_{1}>0$,

$$
\left|\widetilde{r}_{\alpha, j}\left(\mathbf{v}_{j}\right)\right| \leq b_{1} \varepsilon_{j}\left|g_{1, j}\right|^{2}
$$


Note that, if we put

$$
a=2 \lim _{h \rightarrow-\infty} \frac{1}{|h|} \int \frac{d k}{(2 \pi)^{2}} \widehat{g}_{\mathrm{D},+}^{(\geq h)}(\mathbf{k}) \widehat{g}_{\mathrm{D},-}^{(\geq h)}(\mathbf{k})=\frac{\log \gamma}{\pi v_{F}}
$$

where $g_{\mathrm{D}, \omega}^{(\geq h)} \equiv \sum_{j=h}^{0} g_{\mathrm{D}, \omega}^{(j)}$, then

$$
\left|a_{L, \beta}^{(j)}-a\right| \leq C \gamma^{-\left(j-h_{L, \beta}\right)}
$$

Let us now analyze in more detail the functions $r_{\alpha}^{(j)}\left(\mathbf{v}_{j}, \ldots, \mathbf{v}_{0}\right)$, which appear in (2.106). If we define, for $j^{\prime} \geq j+1$,

$$
D_{\alpha}^{\left(j, j^{\prime}\right)}\left(\mathbf{v}_{j}, \ldots, \mathbf{v}_{0}\right)=\beta_{\alpha}^{(j)}\left(\mathbf{v}_{j}, \ldots, \mathbf{v}_{j}, \mathbf{v}_{j^{\prime}}, \ldots, \mathbf{v}_{0}\right)-\beta_{\alpha}^{(j)}\left(\mathbf{v}_{j}, \ldots, \mathbf{v}_{j}, \mathbf{v}_{j}, \ldots, \mathbf{v}_{0}\right)
$$

we can decompose $r_{\alpha}^{(j)}\left(\mathbf{v}_{j}, \ldots, \mathbf{v}_{0}\right)$ in the following way:

$$
r_{\alpha, j}\left(\mathbf{v}_{j}, \ldots, \mathbf{v}_{0}\right)=\sum_{j^{\prime}=j+1}^{0} D_{\alpha}^{\left(j, j^{\prime}\right)}\left(\mathbf{v}_{j}, \ldots, \mathbf{v}_{0}\right)
$$

Note that $D_{\alpha}^{\left(j, j^{\prime}\right)}\left(\mathbf{v}_{j}, \ldots, \mathbf{v}_{0}\right)$ is obtained from $\beta_{\alpha}^{(j)}\left(\mathbf{v}_{j}, \ldots, \mathbf{v}_{0}\right)$, by changing the values of the r.c.c. in the following way: the r.c.c. of scale lower than $j^{\prime}$ are put equal to the corresponding r.c.c. of scale $j$; those of scale greater than $j^{\prime}$ are left unchanged; at least one of the r.c.c. $v_{r, j^{\prime}}$ is substituted with $v_{r, j^{\prime}}-v_{r, j}$. By using the stability property (remark 2 after (2.96), we can show that, if $\varepsilon_{j}$ is small enough,

$$
\left|D_{\alpha}^{\left(j, j^{\prime}\right)}\left(\mathbf{v}_{j}, \ldots, \mathbf{v}_{0}\right)\right| \leq b_{3} \varepsilon_{j} \gamma^{-\left(j^{\prime}-j\right) \vartheta}\left|\mathbf{v}_{j^{\prime}}-\mathbf{v}_{j}\right|
$$

for some $b_{3}>0$. If we insert in the flow equation (2.98) the equations (2.104), (2.106), (2.109), (2.114) and use the bounds (2.105), (2.108), (2.110), (2.112) and (2.115), we get, if $\varepsilon_{j}$ is small enough,

$$
\begin{aligned}
\left|\mathbf{v}_{j-1}-\mathbf{v}_{j}\right| & \leq\left(a+b_{1} \varepsilon_{j}\right)\left|g_{1, j}\right|^{2}+\left(\bar{c} \varepsilon_{0}+b_{2} \varepsilon_{j}^{2}\right) \gamma^{\vartheta j}+b_{2} \varepsilon_{j}^{2} \gamma^{-\left(j-h_{L, \beta}\right)}+ \\
& +b_{3} \varepsilon_{j} \sum_{j^{\prime}=j+1}^{0} \gamma^{-\vartheta\left(j^{\prime}-j\right)}\left|\mathbf{v}_{j^{\prime}}-\mathbf{v}_{j}\right|
\end{aligned}
$$

for some $b_{2}>0$. The form of this bound implies that, in order to control the flow, it is sufficient to prove that $g_{1, j}$ goes to 0 as $j \rightarrow-\infty$ so fast that $\left|g_{1, j}\right|^{2}$ is summable on $j$. Hence, we have to look more carefully to the flow equation of $g_{1, j}$. By proceeding as before, we can write

$$
\begin{gathered}
g_{1, j-1}=g_{1, j}-a g_{1, j}^{2}+\widetilde{r}_{1, j}+r_{1, j}+\bar{r}_{1, j} \\
\left|\widetilde{r}_{1, j}\right| \leq b_{1} \varepsilon_{j}\left|g_{1, j}\right|^{2}, \quad\left|r_{1, j}\right| \leq b_{3} \varepsilon_{j} \sum_{\substack{j^{\prime}=j+1 \\
0}}^{0} \gamma^{-\vartheta\left(j^{\prime}-j\right)}\left|\mathbf{v}_{j^{\prime}}-\mathbf{v}_{j}\right| \\
\left|\bar{r}_{1, j}\right| \leq b_{2} \varepsilon_{j}\left|g_{1, j}\right|\left[\gamma^{\vartheta j}+\gamma^{-\left(j-h_{L, \beta}\right)}\right]
\end{gathered}
$$

where, in the bound of $\bar{r}_{1, j}$, we used the fact that, for symmetry reasons, $b_{1,0}^{(j)}\left(\mathbf{v}_{j}\right)=0$.

The proof that, if $g_{1,0} \in D_{\varepsilon_{0}, \delta}, g_{1, j}$ goes to 0 as $j \rightarrow-\infty$ so fast that $\left|g_{1, j}\right|^{2}$ is summable on $j$, uniformly in $L$ and $\beta$, would be rather simple if $\bar{r}_{1, j}=0$. This is not true, hence we have to show that its contribution is in any case negligible; however, this claim looks reasonable only if both $|j|$ and $j-h_{L, \beta}$ are large enough. To control the "small" values of $j$, we use the remark 
that, as it is easy to show, if $\varepsilon_{0}$ is small enough, there is a constant $c_{4}$, such that, if $g_{1,0} \in D_{\varepsilon_{0}, \delta}$ and $c_{4}\left|j_{0}\right|\left|g_{1,0}\right|^{2} \leq\left|g_{1,0}\right|^{2-\eta}, \eta<1$, then, for $j \geq j_{0}$,

$$
g_{1, j} \in D_{2 \varepsilon_{0}, \delta / 2}, \quad\left|g_{1,0}\right| / 2 \leq\left|g_{1, j}\right| \leq 2\left|g_{1,0}\right|, \quad \varepsilon_{j} \leq 2 \varepsilon_{0}
$$

Hence we put $j_{0}=-\left(c_{4}\left|g_{1,0}\right|^{1 / 2}\right)^{-1}$ and suppose $\varepsilon_{0}$ so small that

$$
\varepsilon_{j_{0}} \gamma^{\frac{\vartheta}{2} j_{0}} \leq 2 c_{5}\left|g_{1, j_{0}}\right| \gamma^{\frac{\vartheta}{2} j_{0}} \leq\left|g_{1, j_{0}}\right|^{3}
$$

where we also used the fact that, since $\hat{v}\left(2 p_{F}\right)>0, \varepsilon_{0} \leq c_{5}\left|g_{1,0}\right|$, for some constant $c_{5}$.

Lemma 2.4 If $g_{1,0} \in D_{\varepsilon_{0}, \delta}$ and $j \geq j_{0}$, then, if $\varepsilon_{0}$ is small enough,

$$
\left|\mathbf{v}_{j-1}-\mathbf{v}_{j}\right| \leq 2 a\left|g_{1, j}\right|^{2}+2 \bar{c} \varepsilon_{0} \gamma^{\frac{\vartheta}{2} j}+2 b_{2} \varepsilon_{j}^{2} \gamma^{-\left(j-h_{L, \beta}\right)}
$$

Proof - We shall proceed by induction. By (2.119), if $\varepsilon_{0}$ is small enough, $\bar{c} \varepsilon_{0}+b_{2} \varepsilon_{j}^{2} \leq(3 / 2) \bar{c} \varepsilon_{0}$ and $a+b_{1} \varepsilon_{j} \leq 3 a / 2$; hence, (2.121) is true for $j=0$. Let us suppose that (2.121) is verified for $j>h>0$. By (2.119), if $j \geq h \geq j_{0},\left|g_{1, j}\right| /\left|g_{1, h}\right| \leq 4$; hence, by using (2.116) and (2.121), we get:

$$
\begin{aligned}
& \left|\mathbf{v}_{h-1}-\mathbf{v}_{h}\right| \leq(3 / 2) a\left|g_{1, h}\right|^{2}+(3 / 2) \bar{c} \varepsilon_{0} \gamma^{\vartheta h}+b_{2} \varepsilon_{h}^{2} \gamma^{-\left(h-h_{L, \beta}\right)}+ \\
& b_{3} \varepsilon_{h} \sum_{j=h+1}^{0} \gamma^{-\vartheta(j-h)}(j-h)\left\{\max _{h<j^{\prime} \leq j}\left[2 a\left|g_{1, j^{\prime}}\right|^{2}+2 \bar{c} \varepsilon_{0} \gamma^{\frac{\vartheta}{2} j^{\prime}}\right]+2 b_{2} \varepsilon_{j^{\prime}}^{2} \sum_{j^{\prime}=h+1}^{j} \gamma^{-\left(j^{\prime}-h_{L, \beta}\right)}\right\} \\
& \leq\left|g_{1, h}\right|^{2}\left[(3 / 2) a+64 a b_{3} \varepsilon_{0} \sum_{n=0}^{\infty} n \gamma^{-\vartheta n}\right]+\gamma^{\frac{\vartheta}{2} h} \varepsilon_{0}\left[(3 / 2) \bar{c}+4 \bar{c} b_{3} \varepsilon_{0} \sum_{n=0}^{\infty} n \gamma^{-\frac{\vartheta}{2} n}\right]+ \\
& b_{2} \varepsilon_{h}^{2} \gamma^{-\left(h-h_{L, \beta}\right)}\left[1+4 b_{3} \varepsilon_{0} \sum_{n=1}^{\infty} \gamma^{-n}\right]
\end{aligned}
$$

Hence, (2.121) is verified also for $j=h$, if $\varepsilon_{0}$ is small enough.

The previous analysis implies that the flow is essentially trivial up to values of $j$ of order $\left|g_{1,0}\right|^{-1 / 2}$ (or even $\left|g_{1,0}\right|^{-\eta}, 0<\eta<1$ ). Let us now consider the region $j \leq j_{0}$, where the term proportional to $\gamma^{\vartheta j}$ in the bound of $\bar{r}_{1, j}$ is expected to be negligible, thanks to the condition (2.120), so that we can hope to prove that $\left|g_{1, j}\right|$ is decreasing. However, since the the term proportional to $\gamma^{-\left(j-h_{L, \beta}\right)}$ is not negligible for $j-h_{L, \beta}$ "too small", we have to put some restriction on the values of $j$. We choose to restrict the detailed analysis of the flow to the region

$$
j \in\left[j_{0}, h_{L, \beta}^{*}\right], \quad h_{L, \beta}^{*}:=\min \left\{j<j_{0}: \gamma^{-\left(j-h_{L, \beta}\right)} \leq\left|g_{1, j}\right|^{2}\right\}
$$

In this region we write (2.117) in the form

$$
g_{1, j-1}=g_{1, j}-a_{j} g_{1, j}^{2}, \quad a_{j} \equiv a-\frac{\widetilde{r}_{1, j}+r_{1, j}+\bar{r}_{1, j}}{g_{1, j}^{2}}
$$

and we define $A_{j_{0}}=0$ and, for $j<j_{0}$,

$$
A_{j}=\frac{1}{j_{0}-j} \sum_{j^{\prime}=j+1}^{j_{0}} a_{j^{\prime}} \quad \widetilde{g}_{1, j}=\frac{g_{1, j_{0}}}{1+A_{j} g_{1, j_{0}}\left(j_{0}-j\right)}
$$


Lemma 2.5 There are constants $c_{1}, c_{2}, c_{3}$ such that, if $g_{1,0} \in D_{\varepsilon_{0}, \delta}$ and it $\varepsilon_{0}$ is small enough, then the following bounds are satisfied, for all $j \in\left[j_{0}, h_{L, \beta}^{*}\right)$.

$$
\begin{gathered}
\varepsilon_{j} \leq c_{3} \varepsilon_{0} \\
\left|\mathbf{v}_{j}-\mathbf{v}_{j+1}\right| \leq c_{1}\left|g_{1, j+1}\right|^{2} \\
\left|g_{1, j}-\widetilde{g}_{1, j}\right| \leq\left|\widetilde{g}_{1, j}\right|^{3 / 2} \\
\left|a_{j}-a\right| \leq c_{2}\left|g_{1, j_{0}}\right|
\end{gathered}
$$

If $j \in\left[h_{L, \beta}^{*}+1, h_{L, \beta}\right)$, we can only say that

$$
\left|g_{1, j}\right| \leq 2\left|g_{1, h_{L, \beta}^{*}}\right|, \quad \varepsilon_{j} \leq 2 c_{3}
$$

Proof - We shall proceed by induction. By using (2.120), (2.121), (2.122) and (2.119), we see that the bounds (2.125) and (2.126) are satisfied for $j=j_{0}$, if $c_{3} \geq 2, c_{1} \geq 3 a$ and $\left(\bar{c} / c_{5}\right) \varepsilon_{0}+8 b_{2} \varepsilon_{0}^{2} \leq a$. Moreover, $g_{1, j_{0}}=\widetilde{g}_{1, j_{0}}$ and, by proceeding as in the proof of Lemma 2.4 and using (2.120), it is easy to prove that there is a constant $\bar{c}_{2}$, such that

$$
\left|a_{j_{0}}-a\right| \leq \bar{c}_{2}\left|g_{1, j_{0}}\right|
$$

Hence, all the bounds are verified (for $\varepsilon_{0}$ small enough) for $j=j_{0}$, if $c_{1} \geq 3 a, c_{2} \geq \bar{c}_{2}$ and $c_{3} \geq 2$. Suppose that they are verified for $j_{0} \geq j \geq h$.

The validity of (2.127) for $j=h-1$ follows from Prop. B.2 which only rests on the bound (2.128) for $j \geq h$. On the other hand, (2.127) implies that, if $\varepsilon_{0}$ is small enough, $2^{-1}\left|\widetilde{g}_{1, j}\right| \leq\left|g_{1, j}\right| \leq 2\left|\widetilde{g}_{1, j}\right|$; hence, using (2.124), we get, for $j>h$

$$
\left|\frac{g_{1, j}}{g_{1, h}}\right| \leq 4 \frac{\left|1+A_{h} g_{1, j_{0}}\left(j_{0}-h\right)\right|}{\left|1+A_{j} g_{1, j_{0}}\left(j_{0}-j\right)\right|}
$$

Let us now define, as in App. B, $A_{j}=\alpha_{j}+i \beta_{j}, \alpha_{j}=\Re A_{j}$, and suppose that

$$
2 c_{2} \varepsilon_{0} \leq a / 2
$$

so that, by (2.119), $\alpha_{j} \geq a / 2,\left|\beta_{j}\right| \leq 2 c_{2} \varepsilon_{0},\left|A_{j}\right| \leq 3 a / 2$, for $j>h$. By proceeding as in the proof of the bound (B.8) in App. B], we get, if $j>h$ and $\left|\operatorname{Arg} g_{1,0}\right| \leq \pi-\delta, \delta>0$ (so that $\left|\operatorname{Arg} g_{1, j_{0}}\right| \leq \pi-\delta / 2$, see (2.119) $)$,

$$
\left|1+g_{1, j_{0}} \alpha_{j}\left(j_{0}-j\right)\right| \geq \frac{1}{3} \sin (\delta / 2)\left[1+\left|g_{1, j_{0}}\right| \alpha_{j}\left(j_{0}-j\right)\right]
$$

and, if we put $1+A_{j} g_{1, j_{0}}\left(j_{0}-j\right)=1+\alpha_{j} g_{1, j_{0}}\left(j_{0}-j\right)+w_{j}$, we choose $\varepsilon_{0}$ so that

$$
\frac{\left|w_{j}\right|}{\left|1+g_{1, j_{0}} \alpha_{j}\left(j_{0}-j\right)\right|} \leq \frac{6 c_{2} \varepsilon_{0}\left|g_{1, j_{0}}\right|\left(j_{0}-j\right)}{\sin (\delta / 2)\left|g_{1, j_{0}}\right|(a / 2)\left(j_{0}-j\right)}=\frac{12 c_{2} \varepsilon_{0}}{a \sin (\delta / 2)} \leq \frac{1}{2}
$$

Then, by using (2.130), we get

$$
\left|\frac{g_{1, j}}{g_{1, h}}\right| \leq \frac{24}{\sin (\delta / 2)} \frac{1+(3 a / 2)\left|g_{1, j_{0}}\right|\left(j_{0}-h\right) \mid}{1+(a / 2)\left|g_{1, j_{0}}\right|\left(j_{0}-j\right) \mid} \leq C_{\delta}(j-h)
$$

for some constant $C_{\delta}$, only depending on $\delta$ and $a$. Moreover, since $\varepsilon_{h} \leq c_{3} \varepsilon_{0}$, then $\bar{c} \varepsilon_{0}+b_{2} \varepsilon_{h}^{2} \leq$ $2 \bar{c} \varepsilon_{0}$ and $a+b_{1} \varepsilon_{j}+b_{2} \varepsilon_{j}^{2} \leq 2 a$, if

$$
b_{2} c_{3}^{2} \varepsilon_{0} \leq \bar{c}, \quad \text { and } b_{1} c_{3} \varepsilon_{0}+b_{2} c_{3}^{2} \varepsilon_{0}^{2} \leq a
$$


Hence, by using the bounds (2.116), (2.126), (2.120), (2.122), (2.134) and (2.133), we get

$$
\begin{aligned}
\left|\mathbf{v}_{h-1}-\mathbf{v}_{h}\right| & \leq 2 a\left|g_{1, h}\right|^{2}+\frac{\bar{c} \varepsilon_{0}}{c_{5}} \gamma^{-\vartheta\left(j_{0}-h\right)}\left|g_{1, j_{0}}\right|^{2}+c_{1} b_{3} \varepsilon_{h} \sum_{j=h+1}^{0} \gamma^{-\vartheta(j-h)}(j-h) \max _{h<j^{\prime} \leq j}\left|g_{1, j^{\prime}}\right|^{2} \\
& \leq\left|g_{1, h}\right|^{2}\left[2 a+\frac{\bar{c} \varepsilon_{0}}{c_{5}} C_{\delta}^{2} \max _{n \geq 0} \gamma^{-n \vartheta} n^{2}+c_{1} \varepsilon_{h} b_{3} C_{\delta}^{2} \sum_{n=0}^{\infty} \gamma^{-\vartheta n} n^{3}\right]
\end{aligned}
$$

It follows that (2.126) is satisfied also for $j=h$, if

$$
2 a+\frac{\bar{c} \varepsilon_{0}}{c_{5}} C_{\delta}^{2} \max _{n \geq 0} \gamma^{-n \vartheta} n^{2}+2 c_{1} c_{3} \varepsilon_{0} b_{3} C_{\delta}^{2} \sum_{n=0}^{\infty} \gamma^{-\vartheta n} n^{3} \leq c_{1}
$$

Moreover, by using (2.126) and $\left|g_{1, j}\right| \leq 2\left|\widetilde{g}_{1, j}\right|$, we get, for some $b_{4}>0$, only depending on $a$, under the condition (2.131):

$$
\varepsilon_{h-1} \leq \varepsilon_{0}+\sum_{j=h}^{0}\left|\mathbf{v}_{j-1}-\mathbf{v}_{j}\right| \leq \varepsilon_{0}+b_{4} c_{1} \varepsilon_{0}
$$

so that $\varepsilon_{h-1} \leq c_{3} \varepsilon_{0}$, if

$$
1+b_{4} c_{1} \leq c_{3}
$$

The bound for $a_{h-1}-a$ can be done in the same way; it is easy to see that

$$
\left|a_{h-1}-a\right| \leq\left[b_{1} c_{3}+b_{2} c_{3}^{2} \varepsilon_{0} C_{\delta}^{2} \max _{n \geq 0} \gamma^{-n \vartheta} n^{2}+2 c_{1} c_{3} b_{3} C_{\delta}^{2} \sum_{n=0}^{\infty} \gamma^{-\vartheta n} n^{3}\right] \varepsilon_{0}
$$

Hence, (2.128) is verified for $j=h-1$, if

$$
\widetilde{c}_{2} \equiv 2 \bar{c} C_{\delta}^{2} \max _{n \geq 0} \gamma^{-n \vartheta} n^{2}+2 c_{1} c_{3} b_{3} C_{\delta}^{2} \sum_{n=0}^{\infty} \gamma^{-\vartheta n} n^{3} \leq c_{2}
$$

The conditions (2.131), (2.132), (2.134), (2.135), (2.136) and (2.138) can be all satisfied, by taking, for example, $c_{1}=4 a, c_{3}=1+4 a b_{4}$ and $c_{2}=\max \left\{\bar{c}_{2}, \widetilde{c}_{2}\right\}$, if $\varepsilon_{0}$ is small enough.

We still have to analyze the flow in the region $j \in\left[h_{L, \beta}^{*}, h_{L, \beta}+1\right]$, in order to prove the bounds (2.129). We should again proceed by iteration, but we prefer to explain the idea of the proof, which can be by now easily translated in the longer formal proof.

Let us consider first the flow equation for $g_{1, j}$. In this region it is not convenient to include the term bounded by $b_{2} \varepsilon_{j}\left|g_{1, j}\right| \gamma^{-\left(j-h_{L, \beta}\right)}$ (see (2.118) ) in the definition of $a_{j}$; hence, we decompose $\bar{r}_{1, j}$ as $\bar{r}_{1, j}=\bar{r}_{1, j}^{(\vartheta)}+\bar{r}_{1, j}^{(L, \beta)}$ and we write (2.117) in form

$$
\begin{gathered}
g_{1, j-1}=g_{1, j}-a_{j}^{\prime} g_{1, j}^{2}+\bar{r}_{1, j}^{(L, \beta)}, \quad a_{j}^{\prime} \equiv a-\frac{\widetilde{r}_{1, j}+r_{1, j}+\bar{r}_{1, j}^{(\vartheta)}}{g_{1, j}^{2}} \\
\left|\bar{r}_{1, j}^{(L, \beta)}\right| \leq b_{2} \varepsilon_{j}\left|g_{1, j}\right| \gamma^{-\left(j-h_{L, \beta}\right)}
\end{gathered}
$$

Note that, if $\varepsilon_{j}$ satisfies the second condition in (2.129), $a_{j}^{\prime}$ satisfies a bound like (2.128), so that the term $-a_{j}^{\prime} g_{1, j}^{2}$ has still the effect to lower the value of $\left|g_{1, j}\right|$ as $j$ decreases. This remark can be translated easily in the claim that $\left|g_{1, j}\right|$ can be bounded by the solution of the flow equation

$$
\bar{g}_{1, j-1}=\bar{g}_{1, j}\left[1+2 c_{3} b_{2} \varepsilon_{0} \gamma^{-\left(j-h_{L, \beta}\right)}\right], \quad \bar{g}_{1, h_{L, \beta}^{*}}=\left|g_{1, h_{L, \beta}^{*}}\right|
$$


whose solution satisfies, for $\varepsilon_{0}$ small enough, the bound

$$
\bar{g}_{1, h} \leq\left|g_{1, h_{L, \beta}^{*}}\right| \exp \left\{2 c_{3} b_{2} \varepsilon_{0} \sum_{j=h+1}^{h_{L, \beta}^{*}} \gamma^{-\left(j-h_{L, \beta}\right)}\right\} \leq 2\left|g_{1, h_{L, \beta}^{*}}\right|
$$

Let us now consider the other couplings; even in this case we have to separate the term proportional to $b_{2} \varepsilon_{j}^{2} \gamma^{-\left(j-h_{L, \beta}\right)}$ from the others; however it is easy to see, by proceeding as before, that the only consequence is that the bound (2.126) has to be modified as

$$
\left|\mathbf{v}_{j}-\mathbf{v}_{j+1}\right| \leq c_{1}^{\prime}\left|g_{1, j+1}\right|^{2}+c_{1}^{\prime \prime} \varepsilon_{j}^{2} \gamma^{-\left(j-h_{L, \beta}\right)}
$$

so that, if $h \in\left[h_{L, \beta}^{*}, h_{L, \beta}+1\right]$

$$
\left|\mathbf{v}_{h}\right| \leq\left|\mathbf{v}_{h_{L, \beta}^{*}}\right|+c_{1}^{\prime} \sum_{j=h+1}^{h_{L, \beta}^{*}-1}\left|g_{1, j+1}\right|^{2}+c_{1}^{\prime \prime} \sum_{j=h+1}^{h_{L, \beta}^{*}-1} \varepsilon_{j}^{2} \gamma^{-\left(j-h_{L, \beta}\right)}
$$

By using (2.140), (2.125), the inductive hypothesis that $\varepsilon_{j} \leq 2 c_{3} \varepsilon_{0}$ and the fact that, by (2.122), $h_{L, \beta}^{*}-h_{L, \beta}-1 \leq \log \left|g_{1, h_{L, \beta}^{*}}\right|^{-2}$, we get

$$
\left|\mathbf{v}_{h}\right| \leq c_{3} \varepsilon_{0}+2 c_{1}^{\prime}\left|g_{1, h_{L, \beta}^{*}}\right|^{2} \log \left|g_{1, h_{L, \beta}^{*}}\right|^{-2}+4 c_{3}^{2} c_{1}^{\prime \prime} \varepsilon_{0}^{2} \sum_{n=1}^{\infty} \gamma^{-1} \leq 2 c_{3} \varepsilon_{0}
$$

if $\varepsilon_{0}$ is small enough.

We finally show that the running coupling constants are well defined in the zero temperature and thermodynamic limit.

Lemma 2.6 For any fixed sequence $\nu_{h}, h \in\left(h_{L, \beta}, 1\right]$, satisfying (2.99) and any fixed $j \leq 0$, $\lim _{\min \{\beta, L\} \rightarrow \infty} \mathbf{v}_{j}=\overline{\mathbf{v}}_{j}$ does exist; moreover, $\lim _{j \rightarrow-\infty} \overline{\mathbf{v}}_{j}=\mathbf{v}_{-\infty}$ with

$$
\begin{aligned}
g_{2,-\infty} & =g_{2,0}-\frac{1}{2} g_{1,0}+O\left(|\lambda|^{3 / 2}\right)=\left[2 \hat{v}(0)-\hat{v}\left(2 p_{F}\right)\right] \lambda+O\left(|\lambda|^{3 / 2}\right) \\
g_{4,-\infty} & =g_{4,0}+O\left(\lambda^{2}\right)=2 \lambda \hat{v}(0)+O\left(\lambda^{2}\right) \\
\delta_{-\infty} & =O(\lambda)
\end{aligned}
$$

Proof - By applying the localization procedure (see (2.65), (2.67)) to the effective potential $\mathcal{V}^{(0)}(\psi)$, we see that

$$
\mathbf{v}_{0}=\mathbf{v}_{\infty}+\sum_{j=0}^{\infty} \sum_{n=1}^{\infty} \sum_{\tau \in \mathcal{T}_{j, n}} \sum_{\mathbf{P}:\left|P_{v_{0}}\right|=m_{\alpha}} \int_{\Lambda^{M}} d\left(\mathbf{x}_{v_{0}} / \mathbf{x}_{0}\right) K_{\mathcal{L}, \tau, L, \beta, \mathbf{P}}^{(j+1)}\left(\mathbf{x}_{v_{0}}\right)
$$

where $m_{\alpha}=4$, if $\alpha=1,2,4, m_{\alpha}=2$, if $\alpha=\delta, \Lambda=\mathcal{C} \times(-\beta / 2, \beta / 2), \mathbf{x}_{0}$ is an arbitrary fixed point in the set $\mathbf{x}_{v_{0}}, M$ is the number of points in $\mathbf{x}_{v_{0}} / \mathbf{x}_{0}, \mathcal{T}_{j-1, n}$ is the family of trees with scale root $j-1, n$ normal endpoints and no special endpoint. Moreover, $\mathbf{v}_{\infty}$ is the term of order 1 in $\lambda$ and the kernels $K_{\mathcal{L}, \tau, \mathbf{P}, L, \beta}^{(j+1)}\left(\mathbf{x}_{v_{0}}\right)$ are obtained from the kernels (2.42) (where the dependence on $L$ and $\beta$ was hidden) by the procedure described after (2.62). Let us now define

$$
\overline{\mathbf{v}}_{0}=\overline{\mathbf{v}}_{\infty}+\sum_{j=1}^{\infty} \sum_{n=1}^{\infty} \sum_{\tau \in \mathcal{T}_{j-1, n}} \sum_{\mathbf{P}:\left|P_{v_{0}}\right|=m_{\alpha}} \int_{\Lambda_{\infty}^{M}} d\left(\mathbf{x}_{v_{0}} / \mathbf{x}_{0}\right) K_{\mathcal{L}, \tau, \mathbf{P}}^{(j+1)}\left(\mathbf{x}_{v_{0}}\right)
$$


where $\Lambda_{\infty}=\mathbb{Z} \times \mathbb{R}, K_{\mathcal{L}, \tau, \mathbf{P}}^{(j+1)}\left(\mathbf{x}_{v_{0}}\right):=\lim _{\min \{\beta, L\} \rightarrow \infty} K_{\mathcal{L}, \tau, L, \beta, \mathbf{P}}^{(j+1)}\left(\mathbf{x}_{v_{0}}\right), \overline{\mathbf{v}}_{\infty}:=\lim _{\min \{\beta, L\} \rightarrow \infty} \mathbf{v}_{\infty}=$ $\left(2 \lambda \hat{v}(0), 2 \lambda \hat{v}(0), 2 \lambda \hat{v}\left(2 p_{F}\right), \delta_{0}\right)$. We want to prove that

$$
\left|\mathbf{v}_{0}-\overline{\mathbf{v}}_{0}\right| \leq C_{0}|\lambda| \gamma^{h_{L, \beta}}
$$

It is easy to see that a bound of this type is valid for $\left|\mathbf{v}_{\infty}-\overline{\mathbf{v}}_{\infty}\right|$. Hence, if we call $T_{j, L, \beta}$ and $T_{j, \infty}$ the contribution of the trees with scale root $j$ to the sum in (2.144) and (2.145), respectively, the bound (2.146) will be proved, if we prove that

$$
\left|T_{j, L, \beta}-T_{j, \infty}\right| \leq C|\lambda| \gamma^{-j} \gamma^{-\left(j-h_{L, \beta}\right)}
$$

which differs from the dimensional bound of $T_{j, L, \beta}$ and $T_{j, \infty}$ for the factor $\gamma^{-\left(j-h_{L, \beta}\right)} \leq \gamma^{h_{L, \beta}}$. Note that

$$
\begin{array}{r}
\left|T_{j, L, \beta}-T_{j, \infty}\right| \leq \sum_{n=1}^{\infty} \sum_{\tau \in \mathcal{T}_{j-1, n}} \sum_{\mathbf{P}:\left|P_{v_{0}}\right|=m_{\alpha}}\left(\Delta_{j, 1}+\Delta_{j, 2}\right), \text { where } \\
\Delta_{j, 1}:=\int^{*} d\left(\mathbf{x}_{v_{0}} / \mathbf{x}_{0}\right)\left|K_{\mathcal{L}, \tau, \beta, L, \mathbf{P}}^{(j+1)}\left(\mathbf{x}_{v_{0}}\right)-K_{\mathcal{L}, \tau, \mathbf{P}}^{(j+1)}\left(\mathbf{x}_{v_{0}}\right)\right| \\
\Delta_{j, 2}:=\int_{\Lambda^{M}}^{* *} d\left(\mathbf{x}_{v_{0}} / \mathbf{x}_{0}\right)\left|K_{\mathcal{L}, \tau, \beta, L, \mathbf{P}}^{(j+1)}\left(\mathbf{x}_{v_{0}}\right)\right|+\int_{\Lambda_{\infty}^{M}}^{* *} d\left(\mathbf{x}_{v_{0}} / \mathbf{x}_{0}\right)\left|K_{\mathcal{L}, \tau, \mathbf{P}}^{(j+1)}\left(\mathbf{x}_{v_{0}}\right)\right|
\end{array}
$$

where $\int^{*} d\left(\mathbf{x}_{v_{0}} / \mathbf{x}_{0}\right)$ denotes the integration over the rectangle centered in $\mathbf{x}_{0}$ and with sides of length $L / 4$ and $\beta / 4$, while $\int^{* *} d\left(\mathbf{x}_{v_{0}} / \mathbf{x}_{0}\right)$ denotes the integration over the complementary region.

In order to bound $\Delta_{j, 1}$, we note that the difference between the two kernels comes from the oscillating factors $e^{i \overline{\mathbf{k}}_{\eta \eta^{\prime}} \mathbf{x}}$, which appear in the $\mathcal{R}$ operation written in coordinate space (obtained by Fourier transforming (2.65) and (2.67)) and from the differences between $g^{(k)}(\mathbf{x})$ and its $\beta, L \rightarrow \infty$ limit $g_{\infty}^{(k)}(\mathbf{x})$. Regarding the first kind of contributions, we note that the difference between the two kernels can be written as a sum over $O(n)$ terms with at least one factor $e^{i \overline{\mathbf{k}}_{\eta \eta^{\prime}} \mathbf{x}}-1$ associated with a tree vertex; this factor modifies the bound by a factor $\gamma^{-k+h_{L, \beta}}$, where $k$ is the scale of the vertex. Since $k \geq j+1$, the dimensional bound of a single tree is modified by a factor $\gamma^{-j+h_{L, \beta}}$, without modifying the dimensional properties of the sum over the tree expansion. Regarding the second kind of contributions, if we write $g^{(k)}(\mathbf{x})=$ $g_{\infty}^{(k)}(\mathbf{x})+\delta g_{\infty}^{(k)}(\mathbf{x})$, we get the dimensional bounds:

$$
\left|\delta g_{\infty}^{(k)}(\mathbf{x})\right| \leq C \gamma^{k} \gamma^{-\left(k-h_{L, \beta}\right)}, \quad \int_{\substack{|x| \leq \beta / 4 \\\left|x_{0}\right| \leq \beta}} d \mathbf{x}\left|\delta g_{\infty}^{(k)}(\mathbf{x})\right| \leq C \gamma^{-k} \gamma^{-\left(k-h_{L, \beta}\right)}
$$

which differ from the bounds of $g^{(k)}(\mathbf{x})$ by a factor $\gamma^{-k+h_{L, \beta}}$, with $k \geq j$. By using the stability property (see Remark 2 at the end of (2.5), we see that the sum over the tree expansion is modified again by a factor $\gamma^{-j+h_{L, \beta}}$.

In order to bound $\Delta_{j, 2}$, we note that, given any contribution to the one of the kernels, one can select in the spanning tree used to perform the integration (see (2.89) a chain of propagators connecting $\mathbf{x}_{0}$ with a point $\hat{\mathbf{x}}$ at a distance greater that $\min (L, \beta)$, and this produces an extra factor much smaller than $\gamma^{-\left(j-h_{L, \beta}\right)}$ in the bound, in an obvious way. This concludes the proof of (2.147).

We now prove that $\lim _{\min \{\beta, L\} \rightarrow \infty} \mathbf{v}_{j}=\overline{\mathbf{v}}_{j}$ does exist even for $j<0$. By using the notation of 92.5 . we can write, if $h_{L, \beta}<j-1: v_{\alpha, j-1}=v_{\alpha, j}+\beta_{\alpha, L, \beta}^{(j)}(\mathbf{v})$, with $\beta_{\alpha, L, \beta}^{(j)}(\mathbf{v})$ of the form

$$
\beta_{\alpha, L, \beta}^{(j)}(\mathbf{v})=\sum_{n=1}^{\infty} \sum_{\tau \in \mathcal{T}_{j, n}} \sum_{\mathbf{P}:\left|P_{v_{0}}\right|=m_{\alpha}} \int_{\Lambda^{M}} d\left(\mathbf{x}_{v_{0}} / \mathbf{x}_{0}\right) K_{\mathcal{L}, \tau, L, \beta, \mathbf{P}}^{(j+1)}\left(\mathbf{x}_{v_{0}}, \mathbf{v}\right)
$$


We now call $\bar{v}_{\alpha, j}$ the solution of the recurrence equation: $\bar{v}_{\alpha, j-1}=\bar{v}_{\alpha, j}+\beta_{\alpha}^{(j)}(\overline{\mathbf{v}})$, with

$$
\beta_{\alpha}^{(j)}(\overline{\mathbf{v}})=\sum_{n=1}^{\infty} \sum_{\tau \in \mathcal{T}_{j-1}, n} \sum_{\mathbf{P}:\left|P_{v_{0}}\right|=m_{\alpha}} \int_{\Lambda_{\infty}^{M}} d\left(\mathbf{x}_{v_{0}} / \mathbf{x}_{0}\right) K_{\mathcal{L}, \tau, \mathbf{P}}^{(j+1)}\left(\mathbf{x}_{v_{0}}, \overline{\mathbf{v}}\right)
$$

where $K_{\tau, \mathbf{P}}^{(j+1)}\left(\mathbf{x}_{v_{0}}, \mathbf{v}\right)=\lim _{\min \{\beta, L\} \rightarrow \infty} K_{\tau, L, \beta, \mathbf{P}}^{(j+1)}\left(\mathbf{x}_{v_{0}}, \mathbf{v}\right)$. We prove by induction that, if $j<0$ and $\lambda$ is small enough,

$$
\left|\mathbf{v}_{j}-\overline{\mathbf{v}}_{j}\right| \leq \gamma^{-\left(j-h_{L, \beta}\right)}
$$

Note that this bound is not optimal, but it is sufficient for our purposes and very easy to prove. We can write:

$$
\left|v_{\alpha, j-1}-\bar{v}_{\alpha, j-1}\right| \leq\left|v_{\alpha, 0}-\bar{v}_{\alpha, 0}\right|+\sum_{k=j}^{0}\left|\beta_{\alpha, L, \beta}^{(k)}(\mathbf{v})-\beta_{\alpha}^{(k)}(\overline{\mathbf{v}})\right|
$$

The bound (2.153) is an immediate consequence of this inequality and (2.146), if we prove that

$$
\left|\beta_{\alpha, L, \beta}^{(k)}(\mathbf{v})-\beta_{\alpha}^{(k)}(\overline{\mathbf{v}})\right| \leq C|\lambda| \gamma^{-\left(k-h_{L, \beta}\right)}
$$

Let us write

$$
\beta_{\alpha, L, \beta}^{(k)}(\mathbf{v})-\beta_{\alpha}^{(k)}(\overline{\mathbf{v}})=\left[\beta_{\alpha, L, \beta}^{(k)}(\mathbf{v})-\beta_{\alpha, L, \beta}^{(k)}(\overline{\mathbf{v}})\right]+\left[\beta_{\alpha, L, \beta}^{(k)}(\overline{\mathbf{v}})-\beta_{\alpha}^{(k)}(\overline{\mathbf{v}})\right]
$$

The first term can be easily bounded by induction, thanks to the stability property of the tree expansion, while the second term can be bounded as in the proof of (2.147) and taking into account the following facts. If $\alpha \neq \delta$, there is no term of order 1 in $\lambda$ in (2.151) and (2.152), so that we can iterate the bound (2.153), by using it only once in the endpoints of the tree expansion of $\left[\beta_{\alpha, L, \beta}^{(k)}(\mathbf{v})-\beta_{\alpha, L, \beta}^{(k)}(\overline{\mathbf{v}})\right]$. This is not true if $\alpha=\delta$; however, in this case the only terms with $n=1$ in (2.151) and (2.152) depend only on $\mathbf{v}_{0}$ and $\overline{\mathbf{v}}_{0}$, respectively, since they are obtained by contracting on scale $k<0$ the irrelevant term produced on scale 0 by the action of the $\mathcal{R}$ operator. Hence, even in this case, we get a factor $|\lambda|$ in the bound, after the insertion of (2.153).

We still have to prove that $\lim _{j \rightarrow-\infty} \overline{\mathbf{v}}_{j}=\mathbf{v}_{-\infty}$ does exist and satisfies (2.142) and (2.143). The first claim is essentially trivial, since it is obvious that $\overline{\mathbf{v}}$ satisfies Lemma 2.5. and, in particular, this implies that, if $\bar{g}_{1,0} \in D_{\varepsilon, \delta}$, with $\varepsilon$ small enough (how small depending on $\delta$ ), $\bar{g}_{1, j}$ goes to 0 , as $j \rightarrow-\infty$, and $\sum_{j=h}^{0}\left|\bar{g}_{1, j}\right|^{2} \leq C \delta^{-1}|\lambda|$, uniformly in $h$. This is an easy consequence of the condition (2.127) and the condition $\hat{v}\left(2 p_{F}\right)>0$; note that the power $3 / 2$ in the r.h.s. of (2.127) could be replaced by $2-\eta, \eta>0$, but 2 is not allowed. Finally, the form of the flow (2.98) implies also that $\bar{g}_{2, j}, \bar{g}_{4, j}$ and $\bar{\delta}_{j}$ converge, as $j \rightarrow-\infty$, to some limits $g_{2,-\infty}, g_{4,-\infty}$ and $\delta_{-\infty}$ of order $\lambda$, satisfying (2.142) and (2.143).

Let us now suppose that $\lambda$ is a (small) positive number; the previous bounds imply that $\bar{g}_{1, j}>0$, for any $j \leq 0$. The following Lemma will allow us to control the logarithmic corrections to the power law fall-off of the correlations.

Lemma 2.7 There are four sequences $w_{i, h}, \delta_{i, h}, i=1,2, h \leq j_{0}$, such that

$$
\begin{gathered}
\sum_{j=h}^{j_{0}} \bar{g}_{1, j}=\left(1+w_{1, h}\right) \frac{1}{a} \log \left[1+a \bar{g}_{1, j_{0}}\left(j_{0}-h\right)\right]+\delta_{1, h} \\
\sum_{j=h}^{j_{0}}\left[\bar{g}_{2, j}-g_{2,-\infty}\right]=\left(1+w_{2, h}\right) \frac{1}{2 a} \log \left[1+a \bar{g}_{1, j_{0}}\left(j_{0}-h\right)\right]+\delta_{2, h}
\end{gathered}
$$


with

$$
\begin{gathered}
\left|w_{i, h}\right| \leq C \lambda, \quad\left|\delta_{i, h}\right| \leq C \lambda^{1 / 2} \\
\left|w_{i, h-1}-w_{i, h}\right| \leq \frac{C \lambda}{\left[1+a \bar{g}_{1, j_{0}}\left(j_{0}-h\right)\right] \log \left[1+a \bar{g}_{1, j_{0}}\left(j_{0}-h\right)\right]}
\end{gathered}
$$

Proof - Let us put $g_{0}=\bar{g}_{1, j_{0}}$, and $a(s)$ the function of $s \geq 0$, such that $a(s)=a_{j_{0}-n}$, if $n \leq s<n+1$. Then, by using (2.124), (2.127) and (2.128), it is easy to see that

$$
\left|\sum_{j=h}^{j_{0}} \bar{g}_{1, j}-I_{j_{0}-h}\right| \leq C \lambda^{1 / 2}, \quad I_{n}=\int_{0}^{n} d s \frac{g_{0}}{1+g_{0} \int_{0}^{s} d t a(t)}
$$

On the other hand, (2.128) also implies that $a(s)=a+\lambda r(s)$, with $|r(s)| \leq C$; hence

$$
I_{n}=\int_{0}^{n} d s \frac{g_{0}}{1+g_{0} a s}-\lambda \int_{0}^{n} d s \frac{g_{0}^{2} \int_{0}^{s} d t r(t)}{\left[1+g_{0} \int_{0}^{s} d t a(t)\right]\left[1+g_{0} a s\right]}
$$

implying that

$$
\left|I_{n}-\frac{1}{a} \log \left(1+a g_{0} n\right)\right| \leq \frac{4 C \lambda}{a^{2}} \int_{0}^{a g_{0} n} d x \frac{x}{(1+x)^{2}}<\frac{4 C \lambda}{a^{2}} \log \left(1+a g_{0} n\right)
$$

Hence there is a constant $\widetilde{w}_{n}$ such that $I_{n}=\left(1 / a+\widetilde{w}_{n}\right) \log \left(1+a g_{0} n\right)$, with $\left|\widetilde{w}_{n}\right| \leq C \lambda$; this bound, together with the bound in (2.161), proves (2.159) for $i=1$. To prove (2.160), note that

$$
\begin{gathered}
\left|I_{n+1}-I_{n}\right| \leq \int_{n}^{n+1} d s \frac{g_{0}}{1+g_{0} \frac{a}{2} s}=\frac{2}{a} \log \left(1+\frac{a g_{0}}{2+a g_{0} n}\right) \\
I_{n+1}-I_{n}=\left(1 / a+\widetilde{w}_{n+1}\right) \log \left(1+\frac{a g_{0}}{1+a g_{0} n}\right)+\left(\widetilde{w}_{n+1}-\widetilde{w}_{n}\right) \log \left(1+a g_{0} n\right)
\end{gathered}
$$

so that, if $\lambda$ is small enough,

$$
\left|\widetilde{w}_{n+1}-\widetilde{w}_{n}\right| \log \left(1+a g_{0} n\right) \leq\left(\frac{3}{a}+C \lambda\right) \log \left(1+\frac{a g_{0}}{1+a g_{0} n}\right) \leq \frac{4 g_{0}}{1+a g_{0} n}
$$

To prove (2.159) and (2.160) for $i=2$, note that, by (2.109) and Lemma 2.5) if $j \leq j_{0}$,

$$
\begin{aligned}
\bar{g}_{2, j}-g_{2,-\infty} & =\sum_{h=-\infty}^{j}\left[\frac{a}{2}+O(\lambda)\right] g_{1, h}^{2}=\left[\frac{a}{2}+O(\lambda)\right] \int_{|j|}^{\infty} d s \frac{g_{1,0}^{2}}{\left(1+a g_{1,0} s\right)^{2}}+O\left(\widetilde{g}_{1, j}^{3 / 2}\right) \\
& =\left[\frac{1}{2}+O(\lambda)\right] \widetilde{g}_{1, j}+O\left(\widetilde{g}_{1, j}^{3 / 2}\right)
\end{aligned}
$$

Hence, the proof of (2.159) is almost equal to the previous one, while the proof of (2.160) needs a slightly different algebra; we omit the details.

\subsection{The flow of renormalization constants}

The renormalization constant of the free measure satisfies

$$
\frac{Z_{j-1}}{Z_{j}}=1+\beta_{z}^{(j)}\left(\vec{g}_{j}, \delta_{j}, \ldots, \vec{g}_{0}, \delta_{0}\right)+\bar{\beta}_{z}^{(j)}\left(\vec{v}_{j} ; . . . \vec{v}_{0} ; \lambda\right) ;
$$


while the renormalization constants of the densities, for $\alpha=C, S_{i}, S C, T C_{i}$ and $i=1,2$, satisfy the equations

$$
\frac{Z_{j-1}^{(i, \alpha)}}{Z_{j}^{(i, \alpha)}}=1+\beta_{(i, \alpha)}^{(j)}\left(\vec{g}_{j}, \delta_{j}, \ldots, \vec{g}_{0}, \delta_{0}\right)+\bar{\beta}_{(i, \alpha)}^{(j)}\left(\vec{v}_{j} ; . ., \vec{v}_{0} ; \lambda\right) .
$$

In these two formulas, by definition, the $\beta_{t}^{(j)}$ functions, with $t=z$ or $(i, \alpha)$, are given by a sum of multiscale graphs, containing only vertices with r.c.c. $\vec{g}_{h}, \delta_{h}, 0 \geq h \geq j$, modified so that the propagators $g_{\omega}^{(h)}$ and the renormalization constants $Z_{h}, Z_{h}^{(i, \alpha)}, 0 \geq h \geq j$, are replaced by $g_{\mathrm{D}, \omega}^{(h)}, Z_{h}^{(D)}, Z_{h}^{(D, i, \alpha)}$ (the definition of $Z_{h}^{(D, i, \alpha)}$ is analogue to the one of $Z_{h}^{(D)}$ ); the $\bar{\beta}_{t}^{(j)}$ functions contain the correction terms together the remainder of the expansion. Note that, by definition, the constants $Z_{j}^{(D)}$ are exactly those generated by (2.162) and (2.104) with $\bar{\beta}_{z}^{(j)}=\bar{\beta}_{\alpha}^{(j)}=0$. Note also that $\left|\bar{\beta}_{z}^{(j)}\right| \leq C \bar{v}_{j}^{2} \gamma^{\vartheta j}$, while $\left|\bar{\beta}_{(i, \alpha)}^{(j)}\right| \leq C \bar{v}_{j} \gamma^{\vartheta j}$.

By using (2.162) and (2.163), we can write

$$
\frac{Z_{j-1}^{(1, \alpha)}}{Z_{j-1}}=\frac{Z_{j}^{(1, \alpha)}}{Z_{j}}\left[1+\beta_{z,(1, \alpha)}^{(j)}\left(\vec{g}_{j}, \delta_{j}\right)+\hat{\beta}_{z,(1, \alpha)}^{(j)}\left(\vec{v}_{j} ; . ., \vec{v}_{0} ; \lambda\right)\right]
$$

with $\left|\hat{\beta}_{z,(1, \alpha)}\right| \leq C \bar{v}_{j} \gamma^{\vartheta j}$. If we define $\widetilde{\beta}_{z,(1, \alpha)}^{(j)}(\vec{g}, \delta)$ the value of $\beta_{z,(1, \alpha)}^{(j)}\left(\vec{g}_{j}, \delta_{j} ; \ldots ; \vec{g}_{0}, \delta_{0}\right)$ at $\left(\vec{g}_{i}, \delta_{i}\right)=(\vec{g}, \delta), j \leq i \leq 0$ and $\widetilde{\beta}_{z,(1, \alpha)}^{(j, \leq 1)}(\vec{g}, \delta)$ the sum of its terms of order 0 and 1 in $g_{1, h}$, it turns out that

$$
\left|\widetilde{\beta}_{z,(1, \alpha)}^{(j, \leq 1)}(\vec{g}, \delta)\right| \leq C\left[\max \left\{\left|g_{1}\right|\left|g_{2}\right|,\left|g_{4}\right|,|\delta|\right\}\right]^{2}\left[\gamma^{\vartheta h}+\gamma^{-\left(j-h_{L, \beta}\right)}\right], \quad \text { if } \alpha=C
$$

This bound, as crucial as the analogous bound (2.108), has been proved in 27; see App. C for some detail. The bound (2.165), together with $\sum_{k=j}^{0}\left|g_{1, k}\right|^{2} \leq C|\lambda|$ and the fact that $Z_{h}^{\left(1, S_{i}\right)}=$ $Z_{h}^{(1, C)}$ by the $\mathrm{SU}(2)$ spin symmetry, imply that

$$
\left|\frac{Z_{j}^{(1, \alpha)}}{Z_{j}}-1\right| \leq C\left|\bar{\varepsilon}_{j}^{2}\right|, \quad \alpha=C, S_{i}
$$

Regarding the flow of the other renormalization constants, we can write

$$
Z_{j}^{(t)}=\gamma^{-\eta_{t} j} \hat{Z}_{j}^{(t)}
$$

where $Z_{j}^{(z)}=Z_{j}$ and, by definition,

$$
\eta_{t} \equiv \lim _{j \rightarrow-\infty} \eta_{t, j}:=\log _{\gamma}\left[1+\beta_{t}^{(0, j)}\left(g_{2,-\infty}, g_{4,-\infty}, \delta_{-\infty} ; \ldots ; g_{2,-\infty}, g_{4,-\infty}, \delta_{-\infty}\right)\right]
$$

Note that the exponents $\eta_{t}$ are functions of $\vec{v}_{-\infty}$ only, an observation which will play a crucial role in the following. Moreover, by an explicit first order calculation, we see that

$$
\eta_{t}=\left\{\begin{array}{cl}
\left(2 \pi v_{F}\right)^{-1} g_{2,-\infty}+O\left(\lambda^{2}\right) & t=(2, C),\left(2, S_{i}\right) \\
-\left(2 \pi v_{F}\right)^{-1} g_{2,-\infty}+O\left(\lambda^{2}\right) & t=(2, S C),\left(2, T C_{i}\right) \\
O\left(\lambda^{2}\right) & \text { otherwise }
\end{array}\right.
$$

while

$$
\frac{\hat{Z}_{h-1}^{(t)}}{\hat{Z}_{h}^{(t)}}=1+O\left(\widetilde{g}_{1, h} \lambda\right)+r_{h}^{(t)}, \quad t=z,(1, \alpha), \alpha \neq T C_{i}
$$




$$
\begin{aligned}
& \frac{\hat{Z}_{h-1}^{(2, C)}}{\hat{Z}_{h}^{(2, C)}}=1-a g_{1, h}+\frac{a}{2}\left(g_{2, h}-g_{2,-\infty}\right)+O\left(\widetilde{g}_{1, h} \lambda\right)+r_{h}^{(2, C)} \\
& \frac{\hat{Z}_{h-1}^{\left(2, S_{i}\right)}}{\hat{Z}_{h}^{\left(2, S_{i}\right)}}=1+\frac{a}{2}\left(g_{2, h}-g_{2,-\infty}\right)+O\left(\widetilde{g}_{1, h} \lambda\right)+r_{h}^{\left(2, S_{i}\right)} \\
& \frac{\hat{Z}_{h-1}^{(2, S C)}}{\hat{Z}_{h}^{(2, S C)}}=1-\frac{a}{2} g_{1, h}-\frac{a}{2}\left(g_{2, h}-g_{2,-\infty}\right)+O\left(\widetilde{g}_{1, h} \lambda\right)+r_{h}^{(2, S C)} \\
& \frac{\hat{Z}_{h-1}^{\left(2, T C_{i}\right)}}{\hat{Z}_{h}^{\left(2, T C_{i}\right)}}=1+\frac{a}{2} g_{1, h}-\frac{a}{2}\left(g_{2, h}-g_{2,-\infty}\right)+O\left(\widetilde{g}_{1, h} \lambda\right)+r_{h}^{\left(2, T C_{i}\right)}
\end{aligned}
$$

where $a$ and $\widetilde{g}_{1, h}$ are defined as in (2.111) and (2.124), respectively, and $\sum_{h=-\infty}^{0}\left|r_{h}^{(t)}\right| \leq C|\lambda|^{2}$. Let us define:

$$
q_{t}^{(h)}=\frac{\log \hat{Z}_{h}^{(t)}}{\log \left(1+a g_{1,0}|h|\right)}
$$

Hence, by using (2.157), (2.158) and (2.159), we get

$$
\begin{aligned}
\left|q_{t}^{(h)}\right| & \leq C \lambda, \quad t=z,(1, \alpha), \alpha \neq T C_{i} \\
\left|q_{t}^{(h)}-\frac{1}{2} \bar{\zeta}_{\alpha}\right| & \leq C \lambda, \quad t=(2, \alpha)
\end{aligned}
$$

where the constants $\bar{\zeta}_{\alpha}$ are those of (1.27).

The existence of the zero temperature and thermodynamic limit can be done exactly as in Lemma 2.6.

\subsection{Flow of $\nu_{h}$ and calculation of $\nu$.}

The sequence $\nu_{j}, h_{L, \beta} \leq j \leq 1$, must satisfy the recursive equation (2.98) with $\alpha=\nu$. If we decompose $\hat{\beta}_{\nu}^{(j)}$ as in (2.104), the function $\beta_{\nu}^{(j)}$ is exactly equal to 0 , because of the oddness of the propagator $g_{D, \omega}^{(h)}$ and the fact that all endpoints are local (those with scale 2 are excluded), hence do not contain oscillator factors. As concerns the function $\bar{\beta}_{\nu}^{(j)}$, in this case we have to extract the contribution of the trees with at least one endpoint of type $\nu$ and we get, if $\vartheta<\vartheta^{\prime}<1$,

$$
\nu_{j-1}=\gamma \nu_{j}+\bar{\beta}_{\nu}^{(j)}=\gamma \nu_{j}+\varepsilon_{j} \sum_{i=j}^{1} \nu_{i} \bar{\beta}_{j, i} \gamma^{-\vartheta^{\prime}(i-j)}+\varepsilon_{j} \gamma^{\vartheta^{\prime} j} \bar{\beta}_{j}, \quad\left|\bar{\beta}_{j, i}\right|,\left|\bar{\beta}_{j}\right| \leq c_{0}
$$

If we iterate this equation, we get $\nu_{h}=\gamma^{-h+1}\left[\nu_{1}+\sum_{j=h+1}^{1} \gamma^{j-2} \bar{\beta}_{\nu}^{(j)}\right]$. We want to show that it is possible to choose $\nu_{1}=\nu$ so that the sequence $\nu_{h}$ solves (2.173) and satisfies, for $\lambda$ small enough, the bound (2.99) (with $\xi$ large enough, see Remark after (2.105)) and $g_{1,0} \in D_{\varepsilon_{0}, \delta}$ (see Lemma 2.4); these are indeed the conditions that allowed us to control the flow of the r.c.c. and the renormalization constants. The choice of $\nu_{1}$ is of course not unique, at finite $L$ and $\beta$, hence we add the constraint that $\nu_{h_{L, \beta}}=0$, so that the sequence $\nu_{h}$ must satisfy the equation

$$
\nu_{h}=-\sum_{j=h_{L, \beta}+1}^{h} \gamma^{-(h-j+1)} \bar{\beta}_{\nu}^{(j)}:=\mathbf{T}(\underline{\nu})_{h}
$$

if $\underline{\nu}$ denotes the sequence $\nu_{h}$. Let us now consider the Banach space $\mathcal{B}_{\vartheta}$ of the sequences $\underline{\nu}$ with norm $\|\underline{\nu}\|_{\vartheta}=\max _{j} \gamma^{-\vartheta j}\left|\nu_{j}\right|$. We want to show that the operator $\mathbf{T}$ is well defined on the closed ball $\mathcal{M}_{\xi}=\left\{\underline{\nu}:\|\underline{\nu}\|_{\vartheta} \leq \xi|\lambda|\right\}$ as a bounded operator $\mathbf{T}: \mathcal{M}_{\xi} \mapsto \mathcal{M}_{\xi}$, if $\xi$ is large enough and $\lambda$ 
is small enough. This implies that the solution of our problem is a fixed point of the operator $\mathbf{T}$ in $\mathcal{M}_{\xi}$ and that this solution does exist and is unique, if we also prove that $\mathbf{T}$ is a contraction on $\mathcal{M}_{\xi}$.

Let us first prove that $\mathbf{T}$ is a bounded operator of $\mathcal{M}_{\xi}$ into $\mathcal{M}_{\xi}$. By using (2.173), we easily see that, if $\underline{\nu} \in \mathcal{M}_{\xi}, \lambda \in D_{\varepsilon_{0}, \delta}$ and $\varepsilon_{j} \leq c_{3} \varepsilon_{0}$, then

$$
\|\mathbf{T}(\underline{\nu})\| \leq c_{3} c_{0} \varepsilon_{0} \sum_{n=0}^{\infty} \gamma^{-\left(1+\vartheta^{\prime}-\vartheta\right) n}\left[1+\xi|\lambda| \sum_{n=0}^{\infty} \gamma^{-\left(\vartheta^{\prime}-\vartheta\right) n}\right]:=c_{2} \varepsilon_{0}\left(1+\bar{c}_{2}|\lambda| \xi\right)
$$

Hence, $\|\mathbf{T}(\underline{\nu})\| \leq \xi|\lambda|$, if $c_{2} \bar{c}_{2} \varepsilon_{0} \leq 1 / 2$ and $\xi \geq 2 c_{2} \varepsilon_{0} /|\lambda|$. The proof that $\mathbf{T}$ is a contraction on $\mathcal{M}_{\xi}$, if $|\lambda|$ is small enough, is a bit more subtle, since now we can not ignore that the r.c.c. and the renormalization constants do depend on $\underline{\nu}$. Let us call $\mathbf{v}_{j}$ and $\mathbf{v}_{j}^{\prime}$ the r.c.c. corresponding to the sequences $\underline{\nu} \in \mathcal{M}_{\xi}$ and $\underline{\nu}^{\prime} \in \mathcal{M}_{\xi}$, respectively; analogously, we shall define $z_{j}=Z_{j-1} / z_{j}-1$ and $z_{j}^{\prime}$. We see immediately that $\max \left\{\left|\mathbf{v}_{0}-\mathbf{v}_{0}^{\prime}\right|,\left|z_{0}-z_{0}^{\prime}\right|\right\} \leq c_{0}|\lambda|\left|\nu_{1}-\nu_{1}^{\prime}\right|$; we shall prove iteratively that there exists $c_{1}>c_{0}$ such that

$$
\max \left\{\left|\mathbf{v}_{h}-\mathbf{v}_{h}^{\prime}\right|,\left|z_{h}-z_{h}^{\prime}\right|\right\} \leq c_{1}|\lambda|\left\|\underline{\nu}-\underline{\nu}^{\prime}\right\|_{\vartheta}
$$

The bound for $z_{h}-z_{h}^{\prime}$ follows easily from that for $\mathbf{v}_{h}-\mathbf{v}_{h}^{\prime}$; hence we shall discuss in some detail only the bound for $\mathbf{v}_{h}-\mathbf{v}_{h}^{\prime}$. Suppose that (2.175) is satisfied for $h \geq j+1$. In order to iterate this bound, we have to control very carefully the flow of the quantity $\Delta_{h}:=g_{1, h}-g_{1, h}^{\prime}$. The result can be easily explained, if one consider the approximate flow of $g_{1, h}$, obtained by substituting the r.h.s. of (2.109) with $-\frac{1}{2} a g_{1, j}^{2}$. In this case we should get

$$
\Delta_{h-1}=\Delta_{h}-\frac{a}{2} \Delta_{h}\left[g_{1, h}+g_{1, h}^{\prime}\right]
$$

which easily implies, by using the bound (2.127) (which is uniform in $\underline{\nu}$ ), that

$$
\left|\Delta_{h}\right| \leq\left|g_{1,0}-g_{1,0}^{\prime}\right| e^{-c_{2} \log (1+\bar{a}|\lambda||h| \mid)} \mid \leq \frac{c_{0}|\lambda|\left|\nu_{1}-\nu_{1}^{\prime}\right|}{(1+\bar{a}|\lambda||h| \mid)^{c_{2}}}
$$

for some positive constants $c_{2}$ and $\bar{a}$. A careful analysis of the real flow can be done by proceeding as in the proof of Lemma 2.4 and Lemma 2.5 of course it involves also the other r.c.c. and has to use the bound (2.175) for $h \geq j+1$ to shows that this bound is correct for $h=j$. One can see that the bound (2.177) is indeed true, if one substitutes $c_{0}$ with some other constant $\bar{c}_{0}>c_{0}$ and $\left|\nu_{1}-\nu_{1}^{\prime}\right|$ with $\left\|\underline{\nu}-\underline{\nu}^{\prime}\right\|_{\vartheta}$, that is

$$
\left|\Delta_{h}\right| \leq \frac{\bar{c}_{0}|\lambda|\left\|\underline{\nu}-\underline{\nu}^{\prime}\right\|_{h}}{(1+\bar{a}|\lambda||h| \mid)^{c_{2}}}
$$

By using (2.98), we can write

$$
\mathbf{v}_{h}-\mathbf{v}_{h}^{\prime}=\mathbf{v}_{0}-\mathbf{v}_{0}^{\prime}+\sum_{j=h+1}^{0}\left[\hat{\beta}^{(j)}-\hat{\beta}^{(j)^{\prime}}\right]
$$

with $\hat{\beta}_{\alpha}^{(j)}=\hat{\beta}_{\alpha}^{(j)}\left(\mathbf{v}_{j}, \ldots, \mathbf{v}_{0} ; \lambda, \nu_{1}\right)$ and $\hat{\beta}_{\alpha}^{(j)^{\prime}}=\hat{\beta}_{\alpha}^{(j)}\left(\mathbf{v}_{j}^{\prime}, \ldots, \mathbf{v}_{0}^{\prime} ; \lambda, \nu_{1}^{\prime}\right)$. Moreover, by analyzing in detail the structure of the functions $\hat{\beta}_{\alpha}^{(j)}$ discussed in 2.6 and the short memory property, one can see that

$$
\left|\hat{\beta}^{(j)}-\hat{\beta}^{(j)^{\prime}}\right| \leq c_{3}\left[\left|\widetilde{g}_{1, j} \Delta_{j}\right|+\left(\left|\widetilde{g}_{1, j}\right|^{2}+\varepsilon_{j} \gamma^{\vartheta j}\right)\left(c_{1}|\lambda|+1\right)\left\|\underline{\nu}-\underline{\nu}^{\prime}\right\|_{\vartheta}\right]
$$

Hence, if $c_{1}|\lambda| \leq 1,\left|\mathbf{v}_{h}-\mathbf{v}_{h}^{\prime}\right| \leq\left|\mathbf{v}_{0}-\mathbf{v}_{0}^{\prime}\right|+c_{4} \varepsilon_{j}\left\|\underline{\nu}-\underline{\nu}^{\prime}\right\|_{\vartheta} \leq c_{5}|\lambda|\left\|\underline{\nu}-\underline{\nu^{\prime}}\right\|_{\vartheta}$. It follows that the bound (2.175) is true for $c_{5}=c_{1}$ and $c_{1}|\lambda| \leq 1$. 
By using (2.174) and (2.173), we have

$$
\begin{aligned}
& \left|\mathbf{T}(\underline{\nu})_{h}-\mathbf{T}\left(\underline{\nu}^{\prime}\right)_{h}\right| \leq \sum_{j=h_{L, \beta}+1}^{h} \gamma^{-(h-j+1)}\left|\beta_{\nu}^{(j)}-\beta_{\nu}^{(j)}\right| \leq \\
& \quad \sum_{j=h_{L, \beta}+1}^{h} \gamma^{-(h-j+1)}\left[c_{1} \gamma^{\vartheta j} \sup _{h}\left|\mathbf{v}_{h}-\mathbf{v}_{h}^{\prime}\right|+c_{2}|\lambda| \gamma^{\vartheta j}\left\|\underline{\nu}-\underline{\nu}^{\prime}\right\|_{\vartheta}\right] \leq c_{3}|\lambda| \gamma^{\vartheta h}\left\|\underline{\nu}-\underline{\nu}^{\prime}\right\|_{\vartheta}
\end{aligned}
$$

Hence, $\mathbf{T}$ is a contraction, if $c_{3}|\lambda|<1$.

\subsection{Calculation of $p_{F}(\bar{\mu}, \lambda)$}

Let us consider the equation (2.174) in the limit $L, \beta \rightarrow \infty$; its solution gives the sequence $\nu_{h}$, whose first element $\nu_{1}$ is the unique value of the function $\nu(\mu, \lambda)$ which allows us to fix at $p_{F}=\arccos \mu$ the value of the interacting Fermi momentum. We want to show that the equation

$$
\bar{\mu}=\mu+\nu(\lambda, \mu)
$$

can be solved with respect to $\mu$ by a function $\mu(\bar{\mu}, \lambda)$, if $\lambda$ is small enough; the interacting Fermi momentum will then be given by $p_{F}(\bar{\mu}, \lambda)=\arccos \mu(\bar{\mu}, \lambda)$. In order to prove this statement, it is of course sufficient to prove that $|\partial \nu / \partial \mu| \leq C|\lambda|$; since $|\mu|<1$, this is equivalent to prove that $\left|\partial \nu / \partial p_{F}\right| \leq C|\lambda|$.

We do not have an explicit expression of $\nu$, but we know that it is equal to the first element $\nu_{1}$ of the sequence $\underline{\nu}$ which uniquely solves the equation (2.174). If we make explicit the dependence of $\mathbf{T}$ on $p_{F}$, we have $\underline{\nu}=\mathbf{T}\left(\underline{\nu}, p_{F}\right)$. Note that the operator $\mathbf{T}\left(\underline{\nu}, p_{F}\right)$ depends on $p_{F}$ explicitly through the kernels appearing in the tree expansion of the functions $\bar{\beta}_{\nu}^{(j)}$ and indirectly trough the r.c.c. $\mathbf{v}_{j}$ :

$$
T_{h}\left(\underline{\nu}, p_{F}\right)=-\sum_{j=-\infty}^{h} \gamma^{-(h-j+1)} \bar{\beta}_{\nu}^{(j)}\left(\mathbf{v}_{j}\left(\underline{\nu}_{\geq j}, p_{F}\right), \ldots, \mathbf{v}_{0}\left(\underline{\nu}_{\geq 0}, p_{F}\right) ; \underline{\nu}_{\geq j}, p_{F}\right)
$$

where $\underline{\nu}_{\geq j}=\left(\nu_{j}, \ldots, \nu_{1}\right)$. Hence, we can calculate the sequence $\underline{\xi}=\partial \underline{\nu} / \partial p_{F}$, by solving the equation

$$
(I-\mathbf{A}) \underline{\xi}=\underline{b}, \quad A_{h, i}=\frac{\partial T_{h}}{\partial \nu_{i}}, \quad b_{h}=\frac{\partial T_{h}}{\partial p_{F}}
$$

The fact that $\left|\partial \nu / \partial p_{F}\right| \leq C|\lambda|$, for $\lambda$ small enough, immediately follows from the following Lemma.

Lemma 2.8 If $\mathcal{B}_{-\eta}$ is defined as after 2.174), then $\underline{b} \in \mathcal{B}_{-\eta}$, for any $\eta \in(0,1)$ (hence the sequence $b_{h}$ can diverge as $\left.h \rightarrow-\infty\right)$ and $\|\underline{b}\|_{-\eta} \leq \bar{c}_{\eta}|\lambda|$, with $\bar{c}_{\eta} \rightarrow \infty$ if $\vartheta \rightarrow 0^{+}$. Moreover, $\mathbf{A}$ is a bounded linear operator on $\mathcal{B}_{-\eta}$, with norm $\|\mathbf{A}\| \leq c_{\eta}|\lambda|$, so that, if $c_{\eta}|\lambda| \leq 1 / 2$, $\underline{\xi} \in \mathcal{B}_{-\eta}$ and $\|\underline{\xi}\|_{-\eta} \leq 2\|\underline{b}\|_{-\eta}$.

Proof - By using (2.183), we get

$$
b_{h}=-\sum_{j=-\infty}^{h} \gamma^{-(h-j+1)}\left[\frac{\partial \bar{\beta}_{\nu}^{(j)}}{\partial p_{F}}+\sum_{k=j}^{0} \frac{\partial \bar{\beta}_{\nu}^{(j)}}{\partial \mathbf{v}_{k}} \frac{\partial \mathbf{v}_{k}}{\partial p_{F}}\right]
$$

Thanks to the short memory property, the bound (2.105) is valid also for $\partial \bar{\beta}_{\nu}^{(j)} / \partial \mathbf{v}_{k}$ with $\varepsilon_{j}$ in place of $\varepsilon_{j}^{2}$. Hence, we get, if $\vartheta<1$ and $\underline{\nu} \in \mathcal{B}_{\vartheta}$,

$$
\left|b_{h}\right| \leq \sum_{j=-\infty}^{h} \gamma^{-(h-j+1)}\left[\left|\frac{\partial \bar{\beta}_{\nu}^{(j)}}{\partial p_{F}}\right|+C|\lambda| \gamma^{\vartheta j} \max _{k \geq j}\left|\frac{\partial \mathbf{v}_{k}}{\partial p_{F}}\right|\right]
$$


In order to evaluate the derivatives with respect to $p_{F}$, note that there is a dependence related to the dependence on $p_{F}$ of the single scale UV propagators $g^{(h)}(\mathbf{x}), h \geq 1$ (see (2.27) ) and the single scale IR propagators $g_{\omega}^{(h)}(\mathbf{x}), h \leq 0$ (see (2.59)); it does not give any trouble, since the bound of $\partial g_{\omega}^{(h)}(\mathbf{x}) / \partial p_{F}$ is similar to that of $g_{\omega}^{(h)}(\mathbf{x})$, as concerns dimensional arguments (even better in the UV case).

In the IR scales there is also a dependence on the oscillator factors $e^{i p_{F} \mathbf{x}}$, which appear on the representation (2.22) of the effective potential $\mathcal{V}^{(0)}$ in terms of the $\psi_{\mathbf{x}, \omega, s}^{ \pm}$fields. In the kernel of a tree this dependence will produce a bad factor $(\mathbf{x}-\mathbf{y})$ multiplying the propagator of scale $j$ joining the points $\mathbf{x}$ and $\mathbf{y}$, hence a factor $\gamma^{-j}$ in the dimensional bound. However, such oscillating factors are not present in the local part of $\mathcal{V}^{(0)}$; they only appear if the tree has at least one endpoint of scale +2 . It follows that

$$
\left|\frac{\partial \bar{\beta}_{\nu}^{(j)}}{\partial p_{F}}\right| \leq C|\lambda|^{2}\left(\gamma^{-(1-\vartheta) j}+\gamma^{\vartheta j}\right) \leq C|\lambda|^{2} \gamma^{-(1-\vartheta) j}
$$

In a similar way we can bound $\partial \mathbf{v}_{k} / \partial p_{F}$. However, since $\mathbf{v}_{k}$ depends on $p_{F}$ also trough $\mathbf{v}_{j}$, $j>k$, we get a diverging contribution also from the trees without oscillating factors. A simple analysis allows us to show, starting from the decomposition of the Beta function (2.104), that

$$
\left|\frac{\partial \mathbf{v}_{j-1}}{\partial p_{F}}\right| \leq\left|\frac{\partial \mathbf{v}_{j}}{\partial p_{F}}\right|+C_{1}|\lambda|^{2} \gamma^{-(1-\vartheta) j}+C_{2}|\lambda| \max _{k \geq j}\left|\frac{\partial \mathbf{v}_{k}}{\partial p_{F}}\right|
$$

which implies the bound

$$
\left|\frac{\partial \mathbf{v}_{j}}{\partial p_{F}}\right| \leq C|\lambda| \gamma^{-(1-\vartheta) j}
$$

If we insert this bound and (2.187) in (2.186), we get that $\left|b_{h}\right| \leq C|\lambda| \gamma^{-(1-\vartheta) h}$.

To complete the proof, we shall now prove that, for any $\vartheta^{\prime} \in(0,1)$,

$$
\left|A_{h, i}\right| \leq C|\lambda| \gamma^{-\vartheta^{\prime}|h-i|}
$$

By using (2.183) and the fact that $\bar{\beta}_{\nu}^{(j)}$ and $\mathbf{v}_{j}$ are independent of $\nu_{i}$ and $\mathbf{v}_{i}$, if $i<j$, we get

$$
A_{h, i}=-\sum_{j=-\infty}^{\min \{i, h\}} \gamma^{-(h-j+1)}\left[\frac{\partial \bar{\beta}_{\nu}^{(j)}}{\partial \nu_{i}}+\sum_{k=j}^{i} \frac{\partial \bar{\beta}_{\nu}^{(j)}}{\partial \mathbf{v}_{k}} \frac{\partial \mathbf{v}_{k}}{\partial \nu_{i}}\right]
$$

By proceeding as in the proof of (2.189), we see that, if $i \geq k$, for any $\vartheta \in(0,1)$,

$$
\left|\frac{\partial \mathbf{v}_{k}}{\partial \nu_{i}}\right| \leq C|\lambda| \gamma^{(1-\vartheta)(i-k)}
$$

On the other hand, by using the properties of $\bar{\beta}_{\nu}^{(j)}$ described before (2.173) and the fact that the only term of order 1 in $\lambda$ does not depend on $\mathbf{v}_{j}, j \leq 0$, we get, if $i \geq j$ and $k \geq j$,

$$
\left|\frac{\partial \bar{\beta}_{\nu}^{(j)}}{\partial \nu_{i}}\right| \leq C|\lambda| \gamma^{-\vartheta(i-j)}, \quad\left|\frac{\partial \bar{\beta}_{\nu}^{(j)}}{\partial \mathbf{v}_{k}}\right| \leq C|\lambda| \gamma^{\vartheta j} \gamma^{-\vartheta(k-j)}
$$

Hence, if $i \leq h$,

$$
\begin{aligned}
\left|A_{h, i}\right| & \leq C|\lambda| \gamma^{-(h-i)} \sum_{j=-\infty}^{i} \gamma^{-(i-j)}\left[\gamma^{-\vartheta(i-j)}+\sum_{k=j}^{i} \gamma^{\vartheta j} \gamma^{-\vartheta(k-j)} \gamma^{(1-\vartheta)(i-k)}\right] \\
& \leq C|\lambda|\left[1+\gamma^{\vartheta i}\right] \gamma^{-(h-i)} \leq C|\lambda| \gamma^{-(h-i)}
\end{aligned}
$$


In the case $i>h$, we have, if $\vartheta>1 / 2$,

$$
\left|A_{h, i}\right| \leq C|\lambda| \sum_{j=-\infty}^{h} \gamma^{-(h-j)}\left[\gamma^{-\vartheta(i-j)}+\gamma^{\vartheta i} \gamma^{(1-2 \vartheta)(i-j)}\right] \leq C|\lambda| \gamma^{-(2 \vartheta-1)(i-h)}
$$

Hence, the bound (2.190) is proved, with $\vartheta^{\prime}=2 \vartheta-1$. The Lemma then follows immediately from (2.190) with $1>\vartheta^{\prime}>\eta$.

\section{Proof of Theorem 1.1}

\subsection{The zero temperature and thermodynamic limit of the free energy}

Note first that the Grassmann integrals for the free energy and the Schwinger functions are analytic in the domain $D$ (2.12), as a consequence of Lemmas 2.2, 2.3, 2.4 and 2.5. therefore, by proposition 2.1, they coincide with the free energy and Schwinger functions of the Hubbard model.

Let us prove first the zero temperature and thermodynamic limit of the free energy $E_{\beta, L}$, which is given (see (2.37) and (2.80) for the notation) by

$$
E=\lim _{\beta \rightarrow \infty} \lim _{L \rightarrow \infty}\left[\sum_{j=h_{L, \beta}}^{0}\left(t_{j}^{(\beta, L)}+\widetilde{E}_{j}^{(\beta, L)}\right)+\sum_{j=1}^{\infty} e_{j}^{(\beta, L)}\right]
$$

We can indeed prove an even stronger result, that is the convergence under the condition that $\min \{\beta, L\} \rightarrow \infty$. In fact, we shall prove that, given $\varepsilon>0$, there exists $h_{\varepsilon}^{*}$, such that, if $\min \{\beta, L\} \geq h_{\varepsilon}^{*}$, then

$$
\left|\sum_{j=h_{L, \beta}}^{0} \widetilde{E}_{j}^{(\beta, L)}-\sum_{j=-\infty}^{0} \widetilde{E}_{j}\right| \leq \varepsilon
$$

where $\widetilde{E}_{j}=\lim _{\min \{\beta, L\} \rightarrow \infty} \widetilde{E}_{j}^{(\beta, L)}$ and this limit does exist, since, by Lemma 2.6, the r.c.c. involved in the tree expansion converge in the same limit, as well as the kernels involved in the definition of $\widetilde{E}_{j}^{(\beta, L)}$, by the same arguments used in the proof of Lemma 2.6.

In order to prove (3.2), we note that, given $\varepsilon>0$, there exists $h_{\varepsilon}$ such that $\mid \sum_{j=h_{L, \beta}}^{h_{\varepsilon}} \widetilde{E}_{j}^{(\beta, L)}-$ $\sum_{j=-\infty}^{h_{\varepsilon}} \widetilde{E}_{j} \mid \leq \varepsilon / 2$, as $\left|\widetilde{E}_{j}^{L, \beta}\right|+\left|\widetilde{E}_{j}\right| \leq C \gamma^{2 j}$, by Lemma 2.3. eq. (2.83) and Lemmas 2.4 and 2.5. Moreover,

$$
\widetilde{E}_{j}^{(\beta, L)}=\sum_{n=1}^{\infty} \sum_{\tau \in \mathcal{T}_{j-1}, n} \sum_{\mathbf{P}: P_{v_{0}}=0} \int_{\Lambda_{\infty}^{M}} d\left(\mathbf{x}_{v_{0}} / \mathbf{x}_{0}\right) K_{\tau, \mathbf{P}}^{(j+1)}\left(\mathbf{x}_{v_{0}}, \overline{\mathbf{v}}\right)
$$

so that, by using Lemma 2.6 and the procedure described in its proof, we get;

$$
\left|\sum_{j=h_{\varepsilon}}^{0} \widetilde{E}_{j}^{(\beta, L)}-\sum_{j=h_{\varepsilon}}^{0} \widetilde{E}_{j}\right| \leq C|\lambda| \gamma^{-\left(h_{\varepsilon}-h_{\varepsilon}^{*}\right)} \leq \frac{\varepsilon}{2}
$$

for $h_{\varepsilon}^{*}$ large enough. This argument can be repeated for $e_{j}$ using that, by Lemma 2.2. $\left|e_{j}\right| \leq C \gamma^{-j}$, while the convergence of the contribution of $t_{j}$ follows immediately from its very definition, see the lines after (2.78). 


\subsection{Tree expansion for the density correlations}

Let us consider now the density correlations. The tree expansion described in $\$ 2.5$ implies that $\Omega_{\alpha}(\mathbf{x}-\mathbf{y})$ can be written as the sum over the values associated with all trees with 2 special endpoints of type $J^{\alpha}$ and fixed space-time points $\mathbf{x}$ and $\mathbf{y}$, a number $n \geq 0$ of normal endpoints and a root of scale $h \leq 0$; moreover, these trees must satisfy the condition that $\left|P_{v_{0}}\right|=0$ (no external legs in the vertex $v_{0}$ of scale $h+1$ following the root), while (as always) $\left|P_{v}\right|>0$ for all other vertices. We shall call $v_{\mathbf{x}}$ and $v_{\mathbf{y}}$ the two special endpoints and $h_{\mathbf{x}}+1, h_{\mathbf{y}}+1$ their scale labels; moreover, we shall denote $v_{\mathbf{x}, \mathbf{y}}$ the higher vertex such that $v_{\mathbf{x}, \mathbf{y}}<v_{\mathbf{x}}, v_{\mathbf{y}}$ and we shall call $h_{\mathbf{x}, \mathbf{y}}$ its scale.

Let un consider for definiteness $\Omega_{C}(\mathbf{x})$. The corresponding trees can be grouped in three classes:

1) the trees with both special end-points associated to the field monomial $\left[Z_{j}^{(1, \alpha)} / Z_{j}\right] O_{\mathbf{x}}^{(1, C)}$ with $j=h_{\mathbf{x}} \leq 0$ or $j=h_{\mathbf{y}} \leq 0$, see (2.74);

$2)$ the trees with both special end-points associated to the field monomial $\left[Z_{j}^{(2, \alpha)} / Z_{j}\right] O_{\mathbf{x}}^{(2, C)}$;

3 ) the other trees, that is those which have at least one special endpoint of scale +2 and those which have both special endpoint of scale $\leq 1$, associated one to $O_{\mathbf{x}}^{(1, C)}$ and the other to $O_{\mathbf{x}}^{(2, C)}$.

If one extracts from the first two classes the trees with no normal endpoints and substitutes in their values the propagators $g_{\omega}^{(h)}(\mathbf{x})$ with their asymptotic expressions $g_{\mathrm{D}, \omega}^{(h)}(\mathbf{x})$, see (2.101), one gets the following expression

$$
\begin{aligned}
\Omega_{C}(\mathbf{x}) & =\Omega^{(1, C)}(\mathbf{x})+\cos \left(2 p_{F} x\right) \Omega^{(2, C)}(\mathbf{x})+\Omega^{(3, C)}(\mathbf{x}) \\
\Omega^{(1, C)}(\mathbf{x}) & =2 \sum_{\omega} \sum_{h, h^{\prime}=h_{L, \beta}}^{0} \frac{\left[Z_{h \vee h^{\prime}}^{(1, C)}\right]^{2}}{Z_{h} Z_{h^{\prime}}} g_{\mathrm{D}, \omega}^{(h)}(\mathbf{x}) g_{\mathrm{D}, \omega}^{\left(h^{\prime}\right)}(\mathbf{x})+\sum_{h=h_{L, \beta}}^{0}\left[\frac{Z_{h}^{(1, C)}}{Z_{h}}\right]^{2} R_{1}^{(h)}(\mathbf{x}) \\
\Omega^{(2, C)}(\mathbf{x}) & =4 \sum_{h, h^{\prime}=h_{L, \beta}}^{0} \frac{\left[Z_{h \vee h^{\prime}}^{(2, C)}\right]^{2}}{Z_{h} Z_{h^{\prime}}} g_{\mathrm{D},+}^{(h)}(\mathbf{x}) g_{\mathrm{D},-}^{\left(h^{\prime}\right)}(\mathbf{x})+\sum_{h=h_{L, \beta}}^{0}\left[\frac{Z_{h}^{(2, C)}}{Z_{h}}\right]^{2} R_{2}^{(h)}(\mathbf{x}) \\
\Omega^{(3, C)}(\mathbf{x}) & =\sum_{h=h_{L, \beta}}^{1} \frac{Z_{h}^{(1, C)} Z_{h}^{(2, C)}}{Z_{h}^{2}} R_{3}^{(h)}(\mathbf{x})
\end{aligned}
$$

where $h \vee h^{\prime}=\max \left\{h, h^{\prime}\right\}$ and the definition of $Z_{h}^{(i, C)}$ has been extended to $h=1$ as $Z_{1}^{(i, C)}=1$. $R_{i}^{(h)}(\mathbf{x}-\mathbf{y})$ is defined, for $i=1,2$ as the sum over all trees of the class $i$ with $n \geq 1$ normal endpoints, such that $h_{\mathbf{x}, \mathbf{y}}=h$ and $h_{r}=h_{L, \beta}-1$, if $h_{r}$ is the scale of the root, plus the corrections to the terms with no normal endpoints. $R_{3}^{(h)}(\mathbf{x}-\mathbf{y})$ is the sum over all trees of the class 3 , such that $h_{\mathbf{x}, \mathbf{y}}=h$ and $h_{r}=h_{L, \beta}-1$.

The functions $R_{i}^{(h)}(\mathbf{x}), i=1,2,3$ have the role of corrections, since we can show that

$$
\begin{gathered}
\left|R_{i}^{(h)}(\mathbf{x}-\mathbf{y})\right| \leq C_{N}\left(|\lambda|+\gamma^{\vartheta h}\right) \frac{\gamma^{2 h}}{1+\left[\gamma^{h}|\mathbf{x}-\mathbf{y}|\right]^{N}} \\
\left|R_{3}^{(h)}(\mathbf{x}-\mathbf{y})\right| \leq C_{N} \frac{\gamma^{2 h} \gamma^{\vartheta h}}{1+\left[\gamma^{h}|\mathbf{x}-\mathbf{y}|\right]^{N}}
\end{gathered}
$$

In order to prove (3.8), let us consider a tree in the tree expansion of $R_{i}^{(h)}(\mathbf{x}-\mathbf{y})$ and note that, given a fixed spanning tree graph $T$ defined as in (2.46), there is a unique path $\mathcal{C}_{\mathbf{x}, \mathbf{y}} \in T$ joining $v_{\mathbf{x}}$ with $v_{\mathbf{y}}$; for each line $l$ of this path, there is a propagator of scale $h_{l} \geq h=h_{\mathbf{x}, \mathbf{y}}$. If one takes into account the effects of the regularization procedure, some of these propagators are derived and join some interpolated points in place of the space-time points associated to the endpoints following $v_{\mathbf{x}, \mathbf{y}}$; however, by using the fact that $\left|g_{\omega}^{(j)}(\mathbf{x})\right| \leq C_{N} \gamma^{j}\left[1+\left(\gamma^{j}|\mathbf{x}|\right)^{N}\right]^{-1}$, 
one can show (see $\$ 5.9$ of 21 ) that one can extract from these chain of propagators at least a decaying factor $C_{N}(2 n+1)^{N}\left[1+\left(\gamma^{j}|\mathbf{x}|\right)^{N}\right]^{-1}$, where $n$ is the number of normal endpoints; note that this bound is trivial in absence of regularization. After this operation, one can bound the sum over all trees as in the proof of (2.83), with two main differences. First of all, one has to perform the sum over the scale indices by fixing $h$ in place of $h_{r}$, but this does not change nothing, since the scaling dimensions of the non trivial vertices are all positive, except that of $v_{0}$, which is 0 . The second difference is that one has to take into account that now two of the space-time points are fixed; hence, in order to perform the integrals over the other points, one can still use the propagators in the spanning tree graphs, but one has to neglect one of them; since the path $\mathcal{C}_{\mathbf{x}, \mathbf{y}}$ always contains at least one propagator of scale $h$, this implies that one gain a factor $\gamma^{2 h}$ with respect to the bound leading to (2.83), which has to be also deprived of the volume factor $L \beta$ and multiplied by the decaying factor. As concerns the factors $Z_{h_{\mathbf{x}}}^{(i, C)} / Z_{h_{\mathbf{x}}}$ and $Z_{h_{\mathbf{y}}}^{(i, C)} / Z_{h_{\mathbf{y}}}$, one can use (2.82) to "change" their scale to $h$, at the price of a innocuous factor $e^{c_{1} \varepsilon_{0}\left[\left(h_{\mathbf{x}}-h\right)+\left(h_{\mathbf{y}}-h\right)\right]}$, which can be distributed along the paths joining $v_{\mathbf{x}}$ and $v_{\mathbf{y}}$ with $v_{\mathbf{x}, \mathbf{y}}$, by slightly modifying the factors $\gamma^{-\left(h_{v}-h_{v^{\prime}}\right)\left[D_{v}+z\left(\left|P_{v}\right|, 1\right)\right]}$ associated to the corresponding non trivial vertices, see (2.93). In the case of the corrections to the leading term, coming from the trees with no endpoints, one has also a factor $\gamma^{\vartheta h}$ coming from the bound (2.102) and Lemma 2.6.

The proof of (3.9) is very similar. One has only to remark that all the trees involved in the tree expansion of $R_{3}^{(h)}(\mathbf{x}-\mathbf{y})$ must have at least an endpoint of scale +2 . This follows from the observation that all field monomials associate to normal endpoints of scale less than 2 contain an even number of field $\psi_{\omega}, \omega= \pm 1$; hence, it is not possible to build a Feynmann graph with no external lines and two source terms, one proportional to $O_{\mathbf{x}}^{(1, C)}$, which has two fields with the same $\omega$, the other proportional to $O_{\mathbf{x}}^{(2, C)}$, which has two fields of opposite $\omega$.

\subsection{Zero temperature and thermodynamic limit for the density corre- lations}

Using the above tree expansion, we can prove the existence of the zero temperature and the thermodynamic limit for the density correlations. Let us consider for definiteness the second term in (3.6) and let us indicate explicitly its $\beta, L$ dependence

$$
\sum_{h=h_{L, \beta}}^{0}\left[\frac{Z_{h}^{(\beta, L)(1, C)}}{Z_{h}^{(\beta, L)}}\right]^{2} R_{1}^{(\beta, L)(h)}(\mathbf{x})
$$

We want to show that, given $\varepsilon>0$, there exists $h_{\varepsilon}^{*}$ such that, if $\min \{\beta, L\} \geq h_{\varepsilon}^{*}$, then

$$
\left|\sum_{h=h_{L, \beta}}^{0}\left[\frac{Z_{h}^{(\beta, L)(1, C)}}{Z_{h}^{(\beta, L)}}\right]^{2} R_{1}^{(\beta, L)(h)}(\mathbf{x})-\sum_{h=-\infty}^{0}\left[\frac{\bar{Z}_{h}^{(1, C)}}{\bar{Z}_{h}}\right]^{2} R_{1}^{(h)}(\mathbf{x})\right| \leq \varepsilon
$$

where, with a notation similar to that used in Lemma 2.6, we write $\lim _{\min \{\beta, L\} \rightarrow \infty} Z_{h}^{(\beta, L)}=\bar{Z}_{h}$ and $\lim _{\min \{\beta, L\} \rightarrow \infty} Z_{h}^{(\beta, L)(1, C)}=Z_{h}^{(1, C)}$, while $R_{1}^{(h)}(\mathbf{x})=\lim _{\min \{\beta, L\} \rightarrow \infty} R_{1}^{(\beta, L)(h)}(\mathbf{x})$; all these limits do exist for the same arguments used in 83.1 . By using that, by (3.8) and the analysis of the ren.c.'s given in $\S 2.7, \frac{\bar{Z}_{h}^{(1, C)}}{Z_{h}} R_{1}^{(h)}(\mathbf{x})$ and $\frac{Z_{h}^{(\beta, L)(1, C)}}{Z_{h}^{(\beta, L)}} R_{1}^{(\beta, L)(h)}(\mathbf{x})$ are bounded by $C \gamma^{2 h}$, there exists $h_{\varepsilon}$ such that

$$
\left|\sum_{h=h_{L, \beta}}^{h_{\varepsilon}}\left[\frac{Z_{h}^{(\beta, L)(1, C)}}{Z_{h}^{(\beta, L)}}\right]^{2} R_{1}^{(\beta, L)(h)}(\mathbf{x})-\sum_{h=-\infty}^{h_{\varepsilon}}\left[\frac{\bar{Z}_{h}^{(1, C)}}{\bar{Z}_{h}}\right]^{2} R_{1}^{(h)}(\mathbf{x})\right| \leq \frac{\varepsilon}{2}
$$


Moreover, by using the tree expansion described before (3.5) and Lemma 2.6. together with the procedure described in its proof, we get

$$
\left|\sum_{h=h_{\varepsilon}}^{0}\left[\frac{Z_{h}^{(\beta, L)(1, C)}}{Z_{h}^{(\beta, L)}}\right]^{2} R_{1}^{(\beta, L)(h)}(\mathbf{x})-\sum_{h=h_{\varepsilon}}^{0}\left[\frac{\bar{Z}_{h}^{(1, C)}}{\bar{Z}_{h}}\right]^{2} R_{1}^{(h)}(\mathbf{x})\right| \leq C \sum_{h=h_{\varepsilon}}^{0} \gamma^{2 h} \gamma^{-\left(h-h_{\varepsilon}^{*}\right)} \leq \frac{\varepsilon}{2}
$$

for $h_{\varepsilon}^{*}$ large enough. We can proceed similarly for all the terms appearing in (3.5), (3.6), (3.7) and this concludes the proof of the existence of the zero temperature and thermodynamic limits for the density correlations

\subsection{Asymptotic behavior of the density correlations}

We want now to discuss how we can derive from the form of the leading terms in (3.6) and (3.7) the leading asymptotic behavior, as described in (1.26). The idea is that, since $\left|g_{\mathrm{D}, \omega}^{(h)}(\mathbf{x})\right| \leq$ $C_{N} \gamma^{h}\left[1+\left(\gamma^{h}|\mathbf{x}|\right)^{N}\right]^{-1}$, if $|\mathbf{x}| \geq 1$, in the sums over $h, h^{\prime}$ of (3.6) and (3.7) the main contribution is given by the terms with $|h|$ and $\left|h^{\prime}\right|$ of the same size as $\log _{\gamma}|\mathbf{x}|$. Hence, one expects that the asymptotic behavior of $\Omega^{(i, C)}(\mathbf{x}), i=1,2$, is the same of the function $\bar{\Omega}^{(i, C)}(\mathbf{x})$, obtained by the substitutions of $\gamma^{-h}$ and $\gamma^{-h^{\prime}}$ with $|\mathbf{x}|$ in the asymptotic expressions of the renormalization constants, given by (2.167) and (2.170), that is

$$
\frac{\left[Z_{h \vee h^{\prime}}^{(i, C)}\right]^{2}}{Z_{h} Z_{h^{\prime}}} \rightarrow|\mathbf{x}|^{2\left(\eta_{i, C}-\eta_{z}\right)}[1+f(\lambda) \log |\mathbf{x}|]^{2\left(q_{i, C}^{\left(h_{\mathbf{x}}\right)}-q_{z}^{\left(h_{x}\right)}\right)}
$$

where the coefficients $q_{t}^{(h)}$ are defined as in (2.171), $h_{\mathbf{x}}=\inf \left\{h: \gamma^{h}|\mathbf{x}| \geq 1\right\}$, and, by (2.73), (2.111), 2.124) and Lemma 2.7.

$$
f(\lambda)=\frac{a g_{1, j_{0}}}{\log \gamma}=\frac{2 \lambda \hat{v}\left(2 p_{F}\right)}{\pi v_{F}}+O\left(\lambda^{3 / 2}\right)
$$

In order to justify the substitution (3.14), let us put $\eta_{i}=2\left(\eta_{i, C}-\eta_{z}\right)$ and $q_{i}(\mathbf{x})$ any continuous interpolation between $2\left[q_{i, C}^{\left(h_{\mathbf{x}}\right)}-q_{z}^{\left(h_{\mathbf{x}}\right)}\right]$ and $2\left[q_{i, C}^{\left(h_{\mathbf{x}}-1\right)}-q_{z}^{\left(h_{\mathbf{x}}-1\right)}\right]$. Note that, thanks to the bounds (2.159) and (2.160), $q_{i}(\mathbf{x})$ is a bounded function of order $\lambda$, defined up to fluctuations bounded, for $|\mathbf{x}| \geq 1$, by $C \lambda[L(\mathbf{x}) \log L(\mathbf{x})]^{-1}$, with $L(\mathbf{x})=1+f(\lambda) \log |\mathbf{x}|$; hence, its precise definition modifies the following expressions only for a factor $1+O(\lambda)$. Let us now note that

$$
\begin{aligned}
& \left|\Omega^{(i, C)}(\mathbf{x})-\bar{\Omega}^{(i, C)}(\mathbf{x})\right| \leq C_{N}|\mathbf{x}|^{\eta_{i}-2}[1+f(\lambda) \log |\mathbf{x}|]^{q_{i}(\mathbf{x})} \sum_{h, h^{\prime}} \frac{\gamma^{h}|\mathbf{x}|}{1+\left(\gamma^{h}|\mathbf{x}|\right)^{N}} \frac{\gamma^{h^{\prime}}|\mathbf{x}|}{1+\left(\gamma^{h^{\prime}}|\mathbf{x}|\right)^{N}} . \\
& .\left|\frac{\left(\gamma^{h}|\mathbf{x}|\right)^{\eta_{z}}\left(\gamma^{h^{\prime}}|\mathbf{x}|\right)^{\eta_{z}}}{\left(\gamma^{h \vee h^{\prime}}|\mathbf{x}|\right)^{2 \eta_{i, C}}}\left[\frac{L(|\mathbf{x}|)}{L\left(\gamma^{|h|} \mid\right)}\right]^{q_{z}^{(h)}}\left[\frac{L(|\mathbf{x}|)}{L\left(\gamma^{\left|h^{\prime}\right|}\right)}\right]^{q_{z}^{\left(h^{\prime}\right)}}\left[\frac{L(|\mathbf{x}|)}{L\left(\gamma^{\left|h \vee h^{\prime}\right|}\right)}\right]^{-2 q_{i, C}^{\left(h \vee h^{\prime}\right)}} \frac{c_{h} c_{h^{\prime}}}{\widetilde{c}_{h \vee h^{\prime}}^{2}}-1\right|
\end{aligned}
$$

where

$$
L(t)=1+f(\lambda) \log t, \quad c_{h}=L\left(\gamma^{|h|}\right)^{q_{z}^{(h)}} / \hat{Z}_{h}^{(z)}, \quad \widetilde{c}_{h}=L\left(\gamma^{|h|}\right)^{-q_{i, C}^{(h)}} / \hat{Z}_{h}^{(i, C)}
$$

By (2.170), (2.157) and (2.158), $c_{h}=1+O\left(\lambda^{1 / 2}\right)$ and $\widetilde{c}_{h}=1+O\left(\lambda^{1 / 2}\right)$. On the other hand, if $r>0$ and $t \neq 0$,

$$
\left|r^{t}-1\right| \leq|t \log r|\left(r^{t}+r^{-t}\right)
$$

and, if $q \neq 0$,

$$
\left|\left[\frac{L(|\mathbf{x}|)}{L\left(\gamma^{|h|}\right)}\right]^{q}-1\right| \leq C_{q}\left[\left|f(\lambda) \log \left(\gamma^{h}|\mathbf{x}|\right)\right|+\left|f(\lambda) \log \left(\gamma^{h}|\mathbf{x}|\right)\right|^{|q|+1}\right]
$$


These two bounds, together with the bound

$$
\sum_{h=-\infty}^{0} \frac{\left(\gamma^{h} r\right)^{\alpha}\left|\log \left(\gamma^{h} r\right)\right|^{\beta}}{1+\left(\gamma^{h} r\right)^{N}} \leq C_{N, \alpha, q}
$$

valid for any $\beta, r>0, a>0$ and $N>\alpha$, imply that

$$
\left|\Omega^{(i, C)}(\mathbf{x})-\bar{\Omega}^{(i, C)}(\mathbf{x})\right| \leq C_{N} \lambda^{1 / 2}|\mathbf{x}|^{\eta_{i}-2}[1+f(\lambda) \log |\mathbf{x}|]^{q_{i}(\mathbf{x})}
$$

By the remark after (2.124), the factor $\lambda^{1 / 2}$ can be improved up to $\lambda^{1-\vartheta}, \vartheta<1$.

In order to complete the proof of (1.26) in the case $\alpha=C$, we have only to calculate $\bar{\Omega}^{(1, C)}(\mathbf{x})$ and $\bar{\Omega}^{(2, C)}(\mathbf{x})$. By using (2.166) , we see that $\eta_{1, C}=\eta_{z}$ and $q_{1, C}^{(h)}=q_{z}^{(h)}$, so that, if we define $X_{2, C}=1-\eta_{2, C}-\eta_{z}$ and $\zeta_{C}(\mathbf{x})=2\left[q_{2, C}(\mathbf{x})-q_{z}(\mathbf{x})\right]$, we get

$$
\begin{aligned}
& \bar{\Omega}^{(1, C)}(\mathbf{x})=2 \sum_{\omega} g_{\mathrm{D}, \omega}(\mathbf{x}) g_{\mathrm{D}, \omega}(\mathbf{x}) \\
& \bar{\Omega}^{(2, C)}(\mathbf{x})=4|\mathbf{x}|^{2\left(1-X_{2, C}\right)}[1+f(\lambda) \log |\mathbf{x}|]^{\zeta_{C}(\mathbf{x})} g_{\mathrm{D},+}(\mathbf{x}) g_{\mathrm{D},-}(\mathbf{x})
\end{aligned}
$$

where $g_{\mathrm{D}, \omega}(\mathbf{x})=\sum_{h=-\infty}^{0} g_{\mathrm{D}, \omega}^{(h)}(\mathbf{x})$. On the other hand, it is easy to see that, for any $N \geq 2$,

$$
g_{\mathrm{D}, \omega}(\mathbf{x})=\frac{1}{2 \pi} \frac{1}{v_{F} x_{0}+i \omega x}+O\left(|\mathbf{x}|^{-N}\right)
$$

It follows that, up to terms of order $|\mathbf{x}|^{-2-\vartheta}$ (as those coming from $\Omega^{3, C}$ ),

$$
\bar{\Omega}^{(1, C)}(\mathbf{x})=\frac{1}{\pi^{2} \widetilde{\mathbf{x}}^{2}} \bar{\Omega}_{0}(\mathbf{x}), \quad \bar{\Omega}^{(2, C)}(\mathbf{x})=\frac{L(\mathbf{x})^{\zeta_{C}(\mathbf{x})}}{\pi^{2}|\widetilde{\mathbf{x}}|^{2 X_{C}}}
$$

where the functions $\bar{\Omega}_{0}(\mathbf{x})$ and $L(\mathbf{x})$ are defined as in Theorem 1.1. The functions $R_{C}(\mathbf{x})$ and $\widetilde{R}_{C}(\mathbf{x})$, appearing in (1.26), are defined in an obvious way in terms of the contributions of order greater than 0 in $\lambda$, which have the same asymptotic behavior of the zero order terms, starting from (3.8) and (3.17). Hence, by using (2.142), (2.169) and (3.15), we get (1.26) for $\alpha=C$, together with the fact that $\zeta_{C}(\mathbf{x})=-3 / 2+O(\lambda)$, in agreement with (1.27), and

$$
X_{C}=1-c \lambda+O\left(\lambda^{2}\right), \quad c=\lim _{\lambda \rightarrow 0} \frac{g_{2,-\infty}}{2 \pi v_{F} \lambda}=\frac{2 \widehat{v}(0)-\widehat{v}\left(2 p_{F}\right)}{2 \pi v_{F}}
$$

Note also that, in Theorem 1.1, we have modified the function $f(\lambda)$ by erasing the terms of order greater than 1 in $\lambda$; the only effect of this modification is a change of the function $\widetilde{R}_{C}(\mathbf{x})$, which does not change its bound.

The proof of (1.26) in the other cases is done in the same way. In particular, in the case $\alpha=S_{i}$ we have to use again the bound (2.166), while the fact that there is no oscillating contribution to the leading term of $\Omega_{T C_{i}}$ is due to the fact there is no local marginal term which can produce it, by the remark after $\$ 2.76$.

\subsection{The two-point function}

Let us now consider the two-point function $S_{2}(\mathbf{x}-\mathbf{y})$. The proof of (1.25) can be done by using the same strategy. In this case, we have to select the trees with two special endpoints of type $\eta$ and fixed space-time points $\mathbf{x}$ and $\mathbf{y}$, the first one associated to the $\eta_{\mathbf{x}}^{-}$field, the second to the $\eta_{\mathbf{y}}^{+}$field; all the other properties, in particular the definition of $v_{\mathbf{x}}, v_{\mathbf{y}}$ and $v_{\mathbf{x}, \mathbf{y}}$ are the same as before. Such trees can be grouped in two classes: the first class contains the trees with both special endpoints of scale $\leq 1$, the second class contains the remaining trees. As before, one can 
see that the second class is associated with terms which decay faster than the leading ones; hence we analyze in detail the trees of the first class and we shall call $\bar{S}_{2}(\mathbf{x}-\mathbf{y})$ their contribution.

If one extracts from the first class the trees with no normal endpoints and substitutes in their values the propagators $g_{\omega}^{(h)}(\mathbf{x})$ with their asymptotic expressions $g_{\mathrm{D}, \omega}^{(h)}(\mathbf{x})$, see (2.101), one gets the following expression:

$$
\bar{S}_{2}(\mathbf{x}-\mathbf{y})=\sum_{\omega} e^{-i \omega p_{F}(x-y)} \sum_{h=h_{L, \beta}}^{0} \frac{1}{Z_{h}} g_{D, \omega}^{(h)}(\mathbf{x}-\mathbf{y})+\sum_{h=h_{L, \beta}}^{0} \frac{1}{Z_{h}} R^{(h)}(\mathbf{x}-\mathbf{y})
$$

where $Z_{h}{ }^{-1} R^{(h)}(\mathbf{x}-\mathbf{y})$ is defined as the sum over all trees with $n \geq 1$ normal endpoints, such that $h_{\mathbf{x}, \mathbf{y}}=h$ and $h_{r}=h_{L, \beta}-1$, if $h_{r}$ is the scale of the root, plus the corrections to the terms with no normal endpoints. By proceeding as in the proof of (3.8), we can show that

$$
\left|R^{(h)}(\mathbf{x}-\mathbf{y})\right| \leq C_{N}\left(|\lambda|+\gamma^{\vartheta h}\right) \frac{\gamma^{h}}{1+\left[\gamma^{h}|\mathbf{x}-\mathbf{y}|\right]^{N}}
$$

The "extraction" of the decaying factor $\left[1+\left[\gamma^{h}|\mathbf{x}-\mathbf{y}|\right]^{N}\right]^{-1}$ is performed exactly as in the proof of (3.8). After this operation, one can bound the sum over all trees as in the proof of (2.83), by taking into account that, in the crucial bound (2.93), the dimensional factor $\gamma^{-D_{v_{0}} h_{r}}=\gamma^{-h_{r}}$ has to be multiplied by a factor $\gamma^{2 h}$ to compensate the "missing integration" (as in the case of $\Omega_{C}(\mathbf{x})$, see above). Since $\gamma^{-h_{r}+2 h}=\gamma^{h} \prod_{v_{0}<v \leq v_{\mathbf{x}, \mathbf{y}}} \gamma^{1}$, this implies that the bound (2.93) has to be modified by substituting the factor $\gamma^{-D_{v_{0}} h_{r}}$ with $\gamma^{h}$ and by adding -1 to the scaling dimension of all vertices belonging to the path which connects $v_{0}$ with $v_{\mathbf{x}, \mathbf{y}}$. Since the dimension of these vertices is $\geq 2$, we can perform without any problem the sum over the scale indices by fixing $h$ in place of $h_{r}$. As concerns the factors $1 / \sqrt{Z_{h_{\mathrm{x}}}}$ and $1 / \sqrt{Z_{h_{\mathrm{y}}}}$ associated to the two special endpoints, one can use (2.82) to "change" their scale to $h$, at the price of a innocuous factor $e^{\frac{1}{2} c_{1} \varepsilon_{0}^{2}\left[\left(h_{\mathbf{x}}-h\right)+\left(h_{\mathbf{y}}-h\right)\right]}$, which can be distributed along the paths joining $v_{\mathbf{x}}$ and $v_{\mathbf{y}}$ with $v_{\mathbf{x}, \mathbf{y}}$, by slightly modifying the factors $\gamma^{-\left(h_{v}-h_{v^{\prime}}\right) D_{v}}$ associated to the corresponding non trivial vertices, see (2.93). In the case of the corrections to the leading term, coming from the trees with no endpoints, one has also a factor $\gamma^{\vartheta h}$ coming from the bound (2.102). By using the same arguments as in the case of $\Omega_{C}(\mathbf{x})$, is an easy exercise to show that (3.20) can be rewritten in the form (1.25). Finally the proof of the existence of the zero temperature and thermodynamic limit of $\bar{S}_{2}(\mathbf{x}-\mathbf{y})$ can be done exactly as for the density correlations.

\subsection{Borel summability}

First consider the free energy $E(\lambda)$ (1.6); we can decompose it as $E(\lambda)=\sum_{h=-\infty}^{0} E_{h}(\lambda)$, where $E_{h}(\lambda)$ is the contribution of the trees whose root has scale $h$, hence depends only on the running couplings $\vec{v}_{j}$ with scale $j>h$. We will show that $E_{h}(\lambda), h \leq 0$ is analytic in the set

$$
D_{\varepsilon, \delta}^{(h)}:=D_{\varepsilon, \delta} \bigcup\left\{\lambda \in \mathbb{C}:|\lambda|<\frac{c_{0}}{1+|h|}\right\}
$$

and such that

$$
\left|E_{h}(\lambda)\right| \leq c_{1} e^{-\kappa|h|}
$$

By using the Lemma at pag. 466 of [24], this property implies that the perturbative expansion of $E(\lambda)$ satisfies the Watson Theorem, see pag. 192 of [28]. Hence it is Borel summable in the usual meaning.

The tree expansion implies that there exists $\varepsilon_{0}$, such that, if

$$
\bar{\lambda}_{h}=\max _{j \geq h}\left|\vec{v}_{j}\right| \leq \varepsilon_{0}
$$


then $\left|E_{h}\right| \leq c_{2} \gamma^{2 h} \varepsilon_{0}$, with $c_{2}$ independent of $h$. The analysis of 2.6 implies that, given $\delta \in$ $(0, \pi / 2)$, there exists $\varepsilon$ such that, if $\lambda \in D_{\varepsilon, \delta}$, the condition (3.24) is verified uniformly in $h$; then it is easy to see that $E(\lambda)$ is analytic in $D_{\varepsilon, \delta}$ and continuous in its closure. The domain of analyticity of $E_{h}(\lambda)$ is in fact larger; the form of the beta function immediately implies that there exist two constants $c_{3}$ and $\bar{c}$ such that $\bar{\lambda}_{0} \leq c_{3}|\lambda|$ and, if $\bar{\lambda}_{h} \leq \varepsilon_{0}$, then $\bar{\lambda}_{h-1} \leq \bar{\lambda}_{h}+\bar{c} \bar{\lambda}_{h}^{2}$; hence, if $c_{3}|\lambda| \leq \min \left\{\varepsilon_{0} / 2,1 /[4 \bar{c}(|h|+1)]\right\}$, then, if $j>h$ and $\bar{\lambda}_{j} \leq 2 \bar{\lambda}_{0}$,

$$
\bar{\lambda}_{j-1} \leq \bar{\lambda}_{0}+|j| \bar{c} \bar{\lambda}_{j}^{2} \leq \bar{\lambda}_{0}\left(1+4|j| \bar{c}_{0}\right) \leq 2 \bar{\lambda}_{0}
$$

It follows that $E_{h}(\lambda)$ is analytic in the set (3.22), with $c_{0}=c_{3}^{-1} \min \left\{\varepsilon_{0} / 2,1 /(4 \bar{c})\right\}$, and that $\left|E_{h}(\lambda)\right| \leq c_{1} \gamma^{2 h}$, with $c_{1}=c_{2} \varepsilon_{0}$; hence $E(\lambda)$ satisfies (3.23) with $\kappa=2 \log \gamma$.

Let us now consider the 2-point Schwinger function $S_{2}(\mathbf{x})$. The discussion in $\$ 3.5$ about its asymptotic behavior implies that we can write $S_{2}(\mathbf{x})=\sum_{h=-\infty}^{0} S_{2, h}(\mathbf{x})$, where $S_{2, h}(\mathbf{x})$ is the contribution of the trees whose root has scale $h$, and we can prove that, if (3.24) is verified (possibly with a smaller $\varepsilon_{0}$ ), then, for any $N>0$,

$$
\left|S_{2, h}(\mathbf{x})\right| \leq c_{N} \sum_{\bar{h}=h+1}^{0} \gamma^{-\frac{\bar{h}-h}{2}} \frac{\gamma^{\bar{h}}}{Z_{\bar{h}}} \frac{1}{1+\left(\gamma^{\bar{h}}|\mathbf{x}|\right)^{N}}, \quad\left|\frac{1}{Z_{h}}\right| \leq \gamma^{\frac{|h|}{4}}
$$

with $c_{N}$ independent of $h$. Hence, if we define $h_{\mathbf{x}}$ so that $\gamma^{h_{\mathbf{x}}}|\mathbf{x}| \in[1, \gamma)$, then, if $h_{\mathbf{x}}>h$

$$
\left|S_{2, h}(\mathbf{x})\right| \leq c_{2}\left[\sum_{\bar{h}=h+1}^{h_{\mathbf{x}}} \gamma^{-\frac{\bar{h}}{2}} \gamma^{\frac{3}{4} \bar{h}} \gamma^{\frac{h}{2}}+\sum_{\bar{h}=h_{\mathbf{x}}}^{0} \gamma^{-\frac{\bar{h}}{2}} \gamma^{\frac{3}{4} \bar{h}} \gamma^{\frac{h}{2}} \gamma^{2\left(h_{\mathbf{x}}-\bar{h}\right)}\right] \leq \widetilde{c}_{2} \gamma^{\frac{h}{2}} \gamma^{\frac{h_{\mathbf{x}}}{4}}
$$

and a similar bound holds for $h_{\mathbf{x}}<h$ so that

$$
\left|S_{2, h}(\mathbf{x})\right| \leq c_{s} \gamma^{\frac{h}{2}}(1+|\mathbf{x}|)^{-1 / 4}
$$

and we can proceed as in free energy case, so proving the analogous of (3.23) for $S_{2}(\mathbf{x})$, with $c_{1}=c_{s}(1+|\mathbf{x}|)^{-1 / 4}$ and $\frac{\kappa}{\log \gamma}=\frac{1}{2}$ (this value could be improved up to any value smaller than 1 , at the price of lowering $\varepsilon_{0}$ down to 0$)$.

A similar argument can be used for the response functions.

\section{A Proof of $(1.20)$}

We have to prove that the r.h.s. of (1.20) and (1.19) are equal; thanks to the antiperiodic condition, we can suppose that $|\tau| \leq \beta / 2$. Since the sum over $k$ is a finite sum and $\chi\left(\gamma^{-M} k_{0}\right)=1$ for $\left|k_{0}\right| \leq \gamma^{M}$, it is sufficient to prove that the function

$$
\Delta_{\beta}(\tau) \equiv \frac{1}{\beta} \sum_{k_{0} \in \mathcal{D}_{\beta}:\left|k_{0}\right| \geq \gamma^{M}} \chi\left(\gamma^{-M} k_{0}\right) \frac{e^{-i k_{0} \tau}}{-i k_{0}+e(k)}
$$

goes to 0 as $M \rightarrow \infty$, if $|\tau| \leq \beta / 2$. Since $\chi(t)=\chi(-t)$, we can write

$$
\begin{aligned}
\Delta_{\beta}(\tau) & =\frac{2}{\beta} \sum_{k_{0} \geq \gamma^{M}} \chi\left(\gamma^{-M} k_{0}\right) \frac{k_{0} \sin \left(k_{0} \tau\right)}{k_{0}^{2}+e(k)^{2}}+\frac{2}{\beta} \sum_{k_{0} \geq \gamma^{M}} \chi\left(\gamma^{-M} k_{0}\right) \frac{e(k) \cos \left(k_{0} \tau\right)}{k_{0}^{2}+e(k)^{2}} \\
& \equiv \Delta_{\beta, 1}(\tau)+\Delta_{\beta, 2}(\tau)
\end{aligned}
$$

Note that $\left|\Delta_{\beta, 2}(\tau)\right| \leq C \int_{\gamma^{M}}^{\gamma^{M+1}} d k_{0} k_{0}^{-2} \leq C \gamma^{-M}$; hence, $\lim _{M \rightarrow \infty} \Delta_{\beta, 2}(\tau)=0$. Moreover, $\Delta_{\beta, 1}(0)=0$ and, if $\tau= \pm \beta / 2, \sin \left(k_{0} \tau\right)= \pm(-1)^{n}$, if $n=\left(\beta k_{0}\right) /(2 \pi)-1 / 2$. Hence, if we put

$$
F_{M}\left(k_{0}\right) \equiv \chi\left(\gamma^{-M} k_{0}\right) \frac{k_{0}}{k_{0}^{2}+e(k)^{2}}, \quad h \equiv \frac{2 \pi}{\beta}
$$


we get

$$
\left|\Delta_{\beta, 1}( \pm \beta / 2)\right| \leq C \int_{\gamma^{M}}^{\infty} d k_{0}\left|\frac{F\left(k_{0}\right)-F\left(k_{0}+h\right)}{h}\right| \leq C \gamma^{-M}
$$

To get a similar bound for $\Delta_{\beta, 1}(\tau), \tau \neq 0, \pm \beta / 2$, we have to use the oscillation properties of $\sin \left(k_{0} \tau\right)$. Note that, if $\sin (h \tau) \neq 0$,

$$
\sin \left(k_{0} \tau\right)=\frac{\cos \left(k_{0} \tau-h \tau\right)-\cos \left(k_{0} \tau+h \tau\right)}{2 \sin (h \tau)}
$$

On the other hand, if $k_{0} \in \mathcal{D}_{\beta}$, the same is true for $k_{0} \pm h$. Hence, if we put $\bar{k}_{0} \equiv \min \left\{k_{0} \geq \gamma^{M}\right\}$, we can write

$$
\begin{gathered}
\Delta_{\beta, 1}(\tau)=\frac{h}{2 \pi \sin (h \tau)}\left[\sum_{k_{0} \geq \gamma^{M}} \cos \left(k_{0} \tau\right)\left[F\left(k_{0}+h\right)-F\left(k_{0}-h\right)\right]+\right. \\
\left.F\left(\bar{k}_{0}\right) \cos \left(\left(\bar{k}_{0}-h\right) \tau\right)-F\left(\bar{k}_{0}-h\right) \cos \left(\bar{k}_{0} \tau\right)\right]
\end{gathered}
$$

so that $\left|\Delta_{\beta, 1}(\tau)\right| \leq C h[2 \pi \sin (h \tau)]^{-1} \gamma^{-M}$.

\section{B The $g_{1}$ map}

Let us consider the following map on the complex plane:

$$
g_{n+1}=g_{n}-a_{n} g_{n}^{2}
$$

where $a_{n}$ is a sequence depending on $g_{0}$, such that, if $\left|g_{0}\right|$ is small enough,

$$
a_{n}=a+\sigma_{n}, \quad\left|\sigma_{n}\right| \leq c_{0}\left|g_{0}\right|
$$

for some positive constants $a$ and $c_{0}$. We want to study the trajectory of the map (B.1), under the condition that

$$
g_{0} \in D_{\varepsilon, \delta}=\{z \in \mathbb{C}:|z|<\varepsilon,|\operatorname{Arg}(z)| \leq \pi-\delta\}, \quad \delta \in(0, \pi / 2)
$$

We shall first study the properties of a sequence $\widetilde{g}_{n}$, which turns out to be a good approximation of $g_{n}$. Let us define:

$$
A_{n}=\frac{1}{n} \sum_{k=0}^{n-1} a_{k}
$$

Lemma B.1 Given $\delta \in(0, \pi / 2)$, there exists $\varepsilon_{0}(\delta)$ such that, if $\varepsilon \leq \varepsilon_{0}(\delta)$ and $g_{0} \in D_{\varepsilon, \delta}$, the sequence

$$
\widetilde{g}_{n}=\frac{g_{0}}{1+g_{0} n A_{n}}
$$

at any step $n \geq 0$ is well defined and does not exit the larger domain $D_{\varepsilon_{1}, \delta_{1}}$, for $\varepsilon_{1}=2 \varepsilon /(\sin \delta)$ and $\delta_{1}=\delta / 2$.

Proof - First of all, we choose $\varepsilon$ so that

$$
c_{0} \varepsilon \leq a / 2 \Rightarrow a / 2 \leq \Re a_{n} \leq 3 a / 2, \quad\left|\Im a_{n}\right| \leq c_{0}\left|g_{0}\right|
$$


where $c_{0}$ is the constant defined in (B.2); we can write

$$
A_{n}=\alpha_{n}+i \beta_{n}, \quad \alpha_{n} \geq a / 2, \quad\left|\beta_{n}\right| \leq c_{0}\left|g_{0}\right| .
$$

Define $\widetilde{z}_{n}:=1+g_{0} n A_{n}:=1+g_{0} n \alpha_{n}+\widetilde{w}_{n}$; then, if $g_{0} \in D_{\varepsilon, \delta}$

$$
\left|1+g_{0} n \alpha_{n}\right| \geq \max \left\{\sin \delta, \frac{\sin \delta}{3}\left(1+\left|g_{0}\right| n \alpha_{n}\right)\right\}
$$

In fact, it is trivial to show that $\left|1+g_{0} n \alpha_{n}\right| \geq \sin \delta$; on the other hand, if $\left|g_{0}\right| n \alpha_{n} \geq 2$,

$$
\left|1+g_{0} n \alpha_{n}\right| \geq\left|g_{0}\right| n \alpha_{n}-1=\left(\left|g_{0}\right| n \alpha_{n}+2\left|g_{0}\right| n \alpha_{n}-3\right) / 3 \geq\left(\left|g_{0}\right| n \alpha_{n}+1\right) / 3
$$

By using (B.8), we get

$$
\frac{\left|\widetilde{w}_{n}\right|}{\left|1+g_{0} n \alpha_{n}\right|} \leq \frac{6 c_{0}}{a \sin \delta}\left|g_{0}\right|
$$

It follows that, if $\varepsilon$ is small enough,

$$
\left|\widetilde{z}_{n}\right| \geq \frac{1}{2} \sin \delta
$$

so that, in particular, the definition (B.5) is meaningful.

Now we want to prove that $\widetilde{g}_{n} \in D_{\varepsilon_{1}, \delta_{1}}$, with $\varepsilon_{1}=2 \varepsilon /(\sin \delta)$ and $\delta_{1}=\delta / 2$, if $\varepsilon$ is small enough. Let $g_{0}=\rho_{0} e^{i \theta_{0}}$; by using (B.8) and (B.9), we see that, if $\varepsilon$ is small enough,

$$
\left|\widetilde{g}_{n}\right| \leq \frac{2\left|g_{0}\right|}{\left|1+\alpha_{n} g_{0} n\right|} \leq \frac{2 \varepsilon}{\sin \delta} ;
$$

besides it is easy to see that

$$
\left|\operatorname{Arg}\left(\frac{g_{0}}{1+\alpha_{n} g_{0} n}\right)\right|=\left|\operatorname{Arg}\left(\frac{\rho_{0}}{e^{-i \theta_{0}}+\alpha_{n} \rho_{0} n}\right)\right| \leq\left|\theta_{0}\right| \leq \pi-\delta .
$$

Then, since $\widetilde{g}_{n}=\frac{\rho_{0}}{e^{-i \theta_{0}}+\alpha_{n} \rho_{0} n}\left(1+w_{n}\right)$, with $w_{n}$ of order $g_{0}$, for $\varepsilon$ small enough,

$$
\left|\operatorname{Arg}\left(\widetilde{g}_{n}\right)\right| \leq \pi-\delta / 2
$$

Proposition B.2 Given $\delta \in(0, \pi / 2)$, there exists $\varepsilon_{0}(\delta)$, such that, if $\varepsilon \leq \varepsilon_{0}(\delta)$ and $g_{0} \in D_{\varepsilon, \delta}$, then

$$
g_{n} \in D_{\varepsilon_{2}, \delta_{2}}, \quad \varepsilon_{2}=\frac{3 \varepsilon}{\sin \delta}, \quad \delta_{2}=\frac{\delta}{4}
$$

Moreover, if $\widetilde{g}_{n}$ is defined as in (B.5),

$$
\left|g_{n}-\widetilde{g}_{n}\right| \leq\left|\widetilde{g}_{n}\right|^{3 / 2}
$$

Proof - We shall proceed by induction on the condition (B.14), which is true for $n=0$. Suppose that it is true for $n \leq N$; then, by using (B.11) and (B.12), we see that, if $\varepsilon$ is small enough and $n \leq N$,

$$
\left|g_{n}\right| \leq 3\left|\widetilde{g}_{n}\right| / 2 \leq 3 \varepsilon / \sin \delta, \quad\left|\operatorname{Arg}\left(g_{n}\right)\right| \leq \pi-\delta / 4
$$

which proves (B.13). Moreover, by (B.1), if $\varepsilon$ is small enough,

$$
\left|g_{N+1}\right| \leq 2\left|g_{N}\right| \leq 3\left|\widetilde{g}_{N}\right|
$$


Note now that

$$
\frac{1}{g_{n+1}}-\frac{1}{g_{n}}=\frac{a_{n}}{1-a_{n} g_{n}}=a_{n}+a_{n}^{2} g_{n}+\Delta_{n}=\frac{1}{\widetilde{g}_{n+1}}-\frac{1}{\widetilde{g}_{n}}+a_{n}^{2} g_{n}+\Delta_{n}
$$

where $\Delta_{n}$ is a quantity which can be bounded by $c_{1}\left|g_{n}\right|^{2}$, for some constant $c_{1}$. We can rewrite (B.17) in the form

$$
\frac{1}{g_{n+1}}-\frac{1}{\widetilde{g}_{n+1}}=\frac{1}{g_{n}}-\frac{1}{\widetilde{g}_{n}}+a_{n}^{2} g_{n}+\Delta_{n}
$$

By using (B.6), (B.8), (B.9), (B.15), (B.16) and (B.18), we get, if $\varepsilon$ is small enough,

$$
\begin{gathered}
\left|g_{N+1}-\widetilde{g}_{N+1}\right|=\left|g_{N+1}\right|\left|\widetilde{g}_{N+1}\right|\left|\frac{1}{g_{N+1}}-\frac{1}{\widetilde{g}_{N+1}}\right| \\
\leq 3\left|\widetilde{g}_{N}\right|\left|\widetilde{g}_{N+1}\right| \sum_{n=0}^{N}\left[6 a^{2}\left|\widetilde{g}_{n}\right|+\frac{9}{4} c_{1}\left|\widetilde{g}_{n}\right|^{2}\right] \leq c_{2}\left|\widetilde{g}_{N}\right|^{3 / 2} \frac{\left|g_{0}\right|^{1 / 2}}{\left(1+\frac{a}{2}\left|g_{0}\right| N\right)^{1 / 2}} \sum_{n=0}^{N} \frac{\left|g_{0}\right|}{1+\frac{a}{2}\left|g_{0}\right| n} \\
\leq\left|\widetilde{g}_{N}\right|^{3 / 2} \frac{c_{3}\left|g_{0}\right|^{1 / 2}}{\left(1+\frac{a}{2}\left|g_{0}\right| N\right)^{1 / 2}} \log \left(1+\frac{a}{2}\left|g_{0}\right| N\right) \leq\left|\widetilde{g}_{N}\right|^{3 / 2}
\end{gathered}
$$

where $c_{2}$ and $c_{3}$ are two suitable constants.

\section{Proof of the partial vanishing of the Beta function}

In this appendix we want to prove the crucial bounds (2.108) and (2.165). This result will be achieved by comparing the beta function of the Hubbard model at the IR scales with that of a reference model, which will be studied in detail in the companion paper [18. This model is built as a perturbation of a Grassmannian-valued Gaussian measure with a two-dimensional continuous field, whose propagator is of the same form as the propagator (2.101) on the IR scales, with $\mathbf{x}$ varying on a continuous square torus; the perturbation is given by an interaction which produces an effective potential with a local part of the same form as that of the Hubbard model, see (2.81), with $\nu_{j}=0$. The point is that, for certain values of the parameters, we can control the beta function of this model by exploiting carefully the local gauge invariance of its interaction. This will be proved in [18; here we discuss how we can use the results of this paper together with the global symmetries of the model to prove (2.108) and (2.165). This strategy is a way to implement the concept of emerging symmetries in a rigorous mathematical setting.

The effective model is expressed in terms of the following Grassmann integral:

$$
\begin{aligned}
e^{\mathcal{W}_{[l, N]}(\eta, J)} & =\int P_{Z}\left(d \psi^{[l, N]}\right) \exp \left\{-\widetilde{V}\left(\sqrt{Z} \psi^{[l, N]}\right)+\sum_{\omega, s} \int_{\Lambda} d \mathbf{x} J_{\mathbf{x}, \omega, s} \psi_{\mathbf{x}, \omega, s}^{[l, N]+} \psi_{\mathbf{x}, \omega, s}^{[l, N]-}\right. \\
& \left.+\sum_{\omega, s} \int_{\Lambda} d \mathbf{x}\left[\psi_{\mathbf{x}, \omega, s}^{[l, N]+} \eta_{\mathbf{x}, \omega, s}^{-}+\eta_{\mathbf{x}, \omega, s}^{+} \psi_{\mathbf{x}, \omega, s}^{[l, N]-}\right]\right\}
\end{aligned}
$$

where $\Lambda$ is a square subset of of size $\widetilde{L}, P_{Z}\left(d \psi^{[l, N]}\right)$ is the fermion measure with propagator

$$
g_{\mathrm{D}, \omega}^{[l, N]}(\mathbf{x})=\frac{1}{Z} \frac{1}{\widetilde{L}^{2}} \sum_{\mathbf{k}} e^{i \mathbf{k x}} \frac{\chi_{l, N}(|\widetilde{\mathbf{k}}|)}{-i k_{0}+\omega c k}, \quad \widetilde{\mathbf{k}}=\left(c k, k_{0}\right), \quad c=v_{F}(1+\delta)
$$

where $Z>0$ and $\delta$ are two parameters and $\chi_{l, N}(t)$ is a smooth compact support function defined for $t \geq 0$, equal to 1 for $\gamma^{l} \leq t \leq \gamma^{N}$ and vanishing for $t \leq \gamma^{l-1}$ or $t \geq \gamma^{N+1}$; $\gamma^{l}$ is the infrared 
cut-off and $\gamma^{N}$ is the ultraviolet cut-off. The limit $N \rightarrow \infty$, followed by the limit $l \rightarrow-\infty$, will be called the limit of removed cut-offs. We choose the cut-off function such that

$$
\chi_{l, N}(|\widetilde{\mathbf{k}}|)=\sum_{j=l}^{N} f_{j}(|\widetilde{\mathbf{k}}|)
$$

with $f_{j}(|\widetilde{\mathbf{k}}|)=\chi\left(\gamma^{j}|\widetilde{\mathbf{k}}|\right)-\chi\left(\gamma^{j+1}(\widetilde{\mathbf{k}})\right.$ and $\chi(t)$ is $C^{\infty}\left(\mathcal{R}^{+}\right)$and such that $\chi(t)=1$ if $t<1 / \gamma$ and $=0$ if $|t|>1$; therefore $f_{j}(|\widetilde{\mathbf{k}}|)$ has non vanishing support in $\gamma^{j-1} \leq|\widetilde{\mathbf{k}}| \leq \gamma^{j+1}$. The interaction is

$$
\widetilde{V}(\psi)=g_{1, \perp} V_{1, \perp}(\psi)+g_{\|} V_{\|}(\psi)+g_{\perp} V_{\perp}(\psi)+g_{4} V_{4}(\psi)
$$

with

$$
\begin{aligned}
V_{1, \perp}(\psi) & =\frac{1}{2} \sum_{\omega, s} \int_{\Lambda} d \mathbf{x} d \mathbf{y} h_{\widetilde{L}}(\mathbf{x}-\mathbf{y}) \psi_{\mathbf{x}, \omega, s}^{+} \psi_{\mathbf{x}, \omega,-s}^{-} \psi_{\mathbf{y},-\omega, s}^{-} \psi_{\mathbf{y},-\omega,-s}^{+} \\
V_{\|}(\psi) & =\frac{1}{2} \sum_{\omega, s} \int_{\Lambda} d \mathbf{x} d \mathbf{y} h_{\widetilde{L}}(\mathbf{x}-\mathbf{y}) \psi_{\mathbf{x}, \omega, s}^{+} \psi_{\mathbf{x}, \omega, s}^{-} \psi_{\mathbf{y},-\omega, s}^{+} \psi_{\mathbf{y},-\omega, s}^{-} \\
V_{\perp}(\psi) & =\frac{1}{2} \sum_{\omega, s} \int_{\Lambda} d \mathbf{x} d \mathbf{y} h_{\widetilde{L}}(\mathbf{x}-\mathbf{y}) \psi_{\mathbf{x}, \omega, s}^{+} \psi_{\mathbf{x}, \omega, s}^{-} \psi_{\mathbf{y},-\omega,-s}^{+} \psi_{\mathbf{y},-\omega,-s}^{-} \\
V_{4}(\psi) & =\frac{1}{2} \sum_{\omega, s} \int_{\Lambda} d \mathbf{x} d \mathbf{y} h_{\widetilde{L}}(\mathbf{x}-\mathbf{y}) \psi_{\mathbf{x}, \omega, s}^{+} \psi_{\mathbf{x}, \omega, s}^{-} \psi_{\mathbf{y}, \omega,-s}^{+} \psi_{\mathbf{y}, \omega,-s}^{-}
\end{aligned}
$$

where $h_{\widetilde{L}}(\mathbf{x})$ is defined in the following way. Let us take a smooth function $\hat{h}(\mathbf{p})$, defined on $\mathbb{R}^{2}$ and rotational invariant, such that $|\hat{h}(\mathbf{p})| \leq C e^{-\mu|\mathbf{p}|}$ for some positive $C$ and $\mu$, and $\hat{h}(0)=1$; moreover, let us call $\mathcal{D}_{\widetilde{L}}$ the set of space-time momenta $\mathbf{k}=\left(k, k_{0}\right)$, with $k=\frac{2 \pi}{\tilde{L}} n$ and $k_{0}=\frac{2 \pi}{\tilde{L}} n_{0}$. Then

$$
h_{\widetilde{L}}(\mathbf{x}):=\frac{1}{\widetilde{L}^{2}} \sum_{\mathbf{p} \in \mathcal{D}_{\tilde{L}}} \hat{h}(\mathbf{p}) e^{i \mathbf{p x}}
$$

We write

$$
g_{\mathrm{D}, \omega}^{[l, N]}(\mathbf{x})=\sum_{j=l}^{N} g_{\mathrm{D}, \omega}^{(j)}(\mathbf{x})
$$

where $g_{\omega}^{(j)}(\mathbf{x})$ is defined as $g_{\omega}^{[l, N]}(\mathbf{x})$ with $\chi_{l, N}(|\widetilde{\mathbf{k}}|)$ replaced by $f_{j}(|\widetilde{\mathbf{k}}|)$, see $[\mathbf{C . 3})$.

The multiscale analysis of [18] shows that, even if the free propagator has the same UV singularity of the Thirring model, the integration of the UV scales is not problematic, since the interaction is not local. As concerns the integration of the infrared scales, it can be done in a way similar to the one in the Hubbard model described in $\$ 2$, which we shall refer to for the notation.

However, before starting the multiscale IR integration, we have to perform some technical operations, which will make possible to compare the flow of the running couplings with that of the Hubbard model. After the integration of the UV scales up to $j=1$, the free measure propagator is given by $g_{\mathrm{D}, \omega}^{[l, 1]}(\mathbf{x})$, defined as in (C.2) with $N=1$. In this expression, the velocity $c$ has the role of the Fermi velocity $v_{F}$ of the Hubbard model. In order to match the asymptotic behavior of the two models, we can not choose $c=v_{F}$; for this reason we introduced the parameter $\delta$. However, it is not possible to compare the RG flows of the two models if the two velocities are different; hence, we have to move from the free measure to the interaction the term proportional to $\delta$. Moreover, since also the cutoff function $\chi^{[l, 1]}\left(\left|\left(k_{0}, c k\right)\right|\right)$ depends on $\delta$, we have to "modify" it in $\chi_{[l, 1]}\left(\left|\left(k_{0}, v_{F} k\right)\right|\right)$. A simple way to perform these operations without introducing spurious 
singularities is described in [18; we shall omit the technical details, which are not important in the following discussion. The final result is that, up to negligible differences for $j=0$ and $j=l$, the effective potential is the same we should get if the propagator of $\psi^{[l, 0]}$ were equal to

$$
\frac{1}{Z} \frac{1}{L^{2}} \sum_{\mathbf{k}} e^{i \mathbf{k x}} \frac{\chi_{l, 0}\left(\left|\left(k_{0}, v_{F} k\right)\right|\right)}{-i k_{0}+\omega v_{F} k}
$$

so that the renormalized single scale propagator will have the form corresponding to the leading behavior of the single scale propagator in the Hubbard model, see (2.100).

Let us now analyze in more detail the RG flow of the effective model for $j \leq 0$. The main difference with respect to the Hubbard model is that (2.71) has to be replaced by

$$
\begin{aligned}
\widetilde{V}^{(j)}\left(\sqrt{Z_{j}} \psi\right) & =g_{1, \perp, j} F_{1, \perp}\left(\sqrt{Z_{j}} \psi\right)+g_{\|, j} F_{\|}\left(\sqrt{Z_{j}} \psi\right)+ \\
+g_{\perp, j} F_{\perp}\left(\sqrt{Z_{j}} \psi\right) & +F_{4}\left(\sqrt{Z_{j}} \psi\right)+\delta_{j} V_{\delta}\left(\sqrt{Z_{j}} \psi\right)
\end{aligned}
$$

where the functions $F_{\alpha}(\psi)$ are defined as the functions $V_{\alpha}(\psi)$ of (C.5) with $\delta(\mathbf{x}-\mathbf{y})$ in place of $h_{L}(\mathbf{x}-\mathbf{y})$; the absence of local terms proportional to $\psi^{+} \psi^{-}$is a consequence of the oddness in $\mathbf{k}$ of the free propagator. The running couplings verify equations of the form

$$
\begin{aligned}
g_{\alpha, h-1}-g_{\alpha, h} & =B_{\alpha}^{(h)}\left(\vec{g}_{h}, \delta_{h}, . . \vec{g}_{0}, \delta_{0}, \vec{g}, \delta\right) \\
\delta_{h-1}-\delta_{h} & =B_{\delta}^{(h)}\left(\vec{g}_{h}, \delta_{h}, . . \vec{g}_{0}, \delta_{0}, \vec{g}, \delta\right)
\end{aligned}
$$

where $\alpha=(1, \perp), \|, \perp, 4$ and $\vec{g}_{j}=\left(g_{1, \perp, j}, g_{\|, j}, g_{\perp, j}, g_{4, j}\right)$. Note that the functions $B_{\alpha}^{(h)}$ and $B_{\delta}^{(h)}$ are of the second order in their arguments; in the case of $B_{\delta}^{(h)}$, this follows from the structure of $\widetilde{V}(\psi)$ (see (C.5)), which does not allow us to build Feynmann graphs of the first order in $\vec{g}$. For the same reason

$$
\delta_{0}=\delta+O\left(\varepsilon_{0}^{2}\right), \quad \varepsilon_{0}=\max \{|\vec{g}|,|\delta|\}
$$

and this relations can be inverted, if $\varepsilon_{0}$ is small enough.

There are some symmetries which is important to exploit. For notational simplicity, we will write $\left(G_{1, \perp}, G_{\|}, G_{\perp}, G_{4}, \Delta\right)$ or $(\vec{G}, \Delta)$ in place of $\left(\vec{g}_{h}, \delta_{h}, . . \vec{g}_{0}, \delta_{0}, \vec{g}, \delta\right)$.

a. Spin $U(1)$. Both the free measure and the interaction are invariant under the transformation

$$
\psi_{\mathbf{x}, \omega, s}^{\varepsilon} \rightarrow e^{i \varepsilon \alpha_{s}} \psi_{\mathbf{x}, \omega, s}^{\varepsilon}
$$

where $\alpha_{s}$ is a spin-dependent angle. This means that the local part of the effective interaction only contains terms which have as many $\psi_{s}^{+}$as $\psi_{s}^{-}$, for each given $s$. Moreover, it is clear from the symmetries $\omega \rightarrow-\omega$ and $s \rightarrow-s$ that all the terms must occur in the same linear combinations of (C.5).

b. Vector-Axial Symmetry. Both the free measure and the interaction are invariant under the transformation

$$
\psi_{\mathbf{x}, \omega, s}^{\varepsilon} \rightarrow e^{i \varepsilon \vartheta_{\omega, s}} \psi_{\mathbf{x}, \omega, s}^{\varepsilon}
$$

with $\alpha_{\omega, s}$ dependent on $\omega$ and $s$. All the interaction terms in (C.5) are invariant but $V_{1, \perp}$. However, if $g_{1, \perp}=0$, it is easy to see, by a graph by graph analysis, that a term of this type can not be generated by the other ones; hence, the function $B_{1, \perp}^{(h)}$ must be odd in $g_{1, \perp}$ :

$$
B_{1, \perp}^{(h)}\left(G_{1, \perp}, G_{\|}, G_{\perp}, G_{4}, \Delta\right)=G_{1, \perp} \bar{B}^{(h)}\left(G_{1, \perp}^{2}, G_{\|}, G_{\perp}, G_{4}, \Delta\right)
$$

where $G_{\alpha}^{2}$ denotes the tensor $\left\{g_{\alpha, j} g_{\alpha, j^{\prime}}\right\}_{j \cdot j^{\prime} \geq h}$ and $G_{\alpha} \bar{B}_{\alpha}^{(h)}$ is a shorthand for $\sum_{j \geq h} g_{\alpha, j} B_{\alpha}^{(h, j)}$. In particular, this implies that, if $g_{1, \perp}=0$, then $g_{1, \perp, j}=0$, that is the surface $\mathcal{C}_{1}=\{\vec{g}, \delta$ : $\left.g_{1, \perp}=0\right\}$, in the space of the interaction parameters $(\vec{g}, \delta)$, is invariant. 
In the same manner, it is easy that the other $B_{\alpha}^{(h)}$ functions are even in $g_{1, \perp}$ :

$$
B_{\alpha}^{(h)}\left(G_{1, \perp}, G_{\|}, G_{\perp}, G_{4}, \Delta\right)=\bar{B}_{\alpha}^{(h)}\left(G_{1, \perp}^{2}, G_{\|}, G_{\perp}, G_{4}, \Delta\right), \quad \alpha=\|, \perp, 4, \delta
$$

c. Spin $S U(2)$. It is convenient to rewrite the interaction as

$$
\begin{aligned}
\widetilde{V}(\psi) & =g_{1, \perp}\left(V_{1, \perp}(\psi)-V_{\|}(\psi)\right)+\left(g_{\|}+g_{1, \perp}-g_{\perp}\right) V_{\|}(\psi)+ \\
& +g_{\perp}\left(V_{\perp}(\psi)+V_{\|}(\psi)\right)+g_{3} V_{3}(\psi)+g_{4} V_{4}(\psi)+\delta_{h} V_{\delta}(\psi)
\end{aligned}
$$

It is evident that $V_{1, \perp}-V_{\|}, V_{\perp}+V_{\|}, V_{3} V_{4}$ and $V_{\delta}$, as well as the free measure, are invariant under the transformation of the fields

$$
\widehat{\psi}_{\mathbf{k}, \omega, s}^{-} \rightarrow \sum_{s^{\prime}} U_{s, s^{\prime}} \widehat{\psi}_{\mathbf{k}, \omega, s^{\prime}}^{-}, \quad \widehat{\psi}_{\mathbf{k}, \omega, s}^{+} \rightarrow \sum_{s^{\prime}} \widehat{\psi}_{\mathbf{k}, \omega, s^{\prime}}^{+} U_{s^{\prime}, s}^{\dagger}
$$

for $U \in S U(2)$. While $V_{\|}$isn't: if $g_{\|}+g_{1}-g_{\perp}=0$ it will remain zero. Thus we find two others invariant surfaces:

$$
C_{1,+}=\left\{\vec{g}, \delta: g_{1, \perp}=g_{\perp}-g_{\|}\right\}, \quad C_{1,-}=\left\{\vec{g}, \delta:-g_{1, \perp}=g_{\perp}-g_{\|}\right\}
$$

Finally we consider the flow of $Z_{h}$ and the renormalization constant $Z_{h}^{(1)}$ associated with the density operator $\rho_{\mathbf{x}, \omega, s}=\psi_{\mathbf{x}, \omega, s}^{+} \psi_{\mathbf{x}, \omega, s}^{-}$in the generating functional (C.1); $Z_{h}^{(1)}$ is defined as $Z_{h}^{(1, C)}$ in (2.74). It is easy to see, by using the symmetry properties of the model as before, that $Z_{h-1} / Z_{h}=1+B_{z}^{(h)}(\vec{G}, \Delta)$ and $Z_{h-1}^{(1)} / Z_{h}^{(1)}=1+B_{\rho}^{(h)}(\vec{G}, \Delta)$, with $B_{\alpha}^{(h)}(\vec{G}, \Delta)=$ $\bar{B}_{\alpha}^{(h)}\left(G_{1}^{2}, G_{3}^{2}, G_{\|}, G_{\perp}, G_{4}, \Delta\right)$ for $\alpha=z, \rho$. Hence

$$
\frac{Z_{h-1}^{(1)}}{Z_{h-1}}=\frac{Z_{h}^{(1)}}{Z_{h}}\left[1+\widetilde{B}^{(h)}(\vec{G}, \Delta)\right]
$$

with

$$
\widetilde{B}^{(h)}(\vec{G}, \Delta)=\bar{B}^{(h)}\left(G_{1, \perp}^{2}, G_{3}^{2}, G_{\|}, G_{\perp}, G_{4}, \Delta\right)
$$

Let us now consider the Hubbard model. In (2.104) we have written its Beta function as sum of two terms, the second of which is asymptotically negligible, by (2.105); the first term, denoted in (2.104) by $\beta_{\alpha}^{(j)}\left(\vec{g}_{j}, \delta_{j} ; \ldots ; \vec{g}_{0}, \delta_{0}\right) \equiv \beta_{\alpha}^{(j)}\left(G_{1}, G_{2}, G_{4}, \Delta\right)$ coincides with the Beta function of the effective model on the invariant surface $\mathcal{C}_{1,+}$, if we subtract from it the contribution of the trees containing endpoints of scale grater than 0 and we interpret everywhere the integrals over the space-time variables, which in the Hubbard model case are a shorthand for $\sum_{x \in \mathcal{C}} \int_{-\beta / 2}^{\beta / 2}$ as the integrals over $\Lambda$, with $\widetilde{L}^{-1}=\max \left\{L^{-1}, \beta^{-1}\right\}$. As discussed in $\S 4.6$ of 21], this modification produces an error of order $\varepsilon_{j}^{2} \gamma^{\vartheta j}$.

Hence, by using (C.13) and (C.12), we get

$$
\begin{aligned}
& \beta_{1}^{(j)}\left(G_{1}, G_{2}, G_{4}, \Delta\right)=G_{1} \bar{\beta}_{1 \perp}^{(j)}\left(G_{1}^{2}, G_{2}-G_{1}, G_{2}, G_{4}, \Delta\right)+O\left(\varepsilon_{j}^{2} \gamma^{\vartheta j}\right) \\
& \beta_{2}^{(j)}\left(G_{1}, G_{2}, G_{4}, \Delta\right)=\bar{\beta}_{\perp}^{(j)}\left(G_{1}^{2}, G_{2}-G_{1}, G_{2}, G_{4}, \Delta\right)+O\left(\varepsilon_{j}^{2} \gamma^{\vartheta j}\right) \\
& \beta_{4}^{(j)}\left(G_{1}, G_{2}, G_{4}, \Delta\right)=\bar{\beta}_{4}^{(j)}\left(G_{1}^{2}, G_{2}-G_{1}, G_{2}, G_{4}, \Delta\right)+O\left(\varepsilon_{j}^{2} \gamma^{\vartheta j}\right) \\
& \beta_{\delta}^{(j)}\left(G_{1}, G_{2}, G_{4}, \Delta\right)=\bar{\beta}_{\delta}^{(j)}\left(G_{1}^{2}, G_{2}-G_{1}, G_{2}, G_{4}, \Delta\right)+O\left(\varepsilon_{j}^{2} \gamma^{\vartheta j}\right)
\end{aligned}
$$

where $\bar{\beta}_{\alpha}^{(j)}\left(G_{1}^{2}, G_{2}-G_{1}, G_{2}, G_{4}, \Delta\right)$ denotes the value of $\bar{B}_{\alpha}^{(j)}\left(G_{1}^{2}, G_{2}-G_{1}, G_{2}, G_{4}, \Delta\right)$, after the subtraction of the trees containing endpoints of scale grater than 0 . 
Therefore, if $\alpha \neq 1$, the contributions of order 0 and 1 in $G_{1}$ of $\beta_{\alpha}^{(j)}(\vec{G}, \Delta)$ are the same as the contributions of the same order of $\bar{\beta}_{\alpha}^{(j)}\left(0, G_{2}-G_{1}, G_{2}, G_{4}, \Delta\right)$. On the other hand, in the following paper, see (4.44) of [18], we will prove that, if we call $b_{\alpha}^{(j)}\left(\bar{g}_{\|}, \bar{g}_{\perp}, \bar{g}_{4}, \bar{\delta}\right)$ the value taken by $\bar{\beta}_{\alpha}^{(j)}\left(0, G_{\|}, G_{\perp}, G_{4}, \Delta\right)$ when $\left(g_{\|, j}, g_{\perp, j}, g_{4, j}, \delta_{j}\right)=\left(\bar{g}_{\|}, \bar{g}_{\perp}, \bar{g}_{4}, \bar{\delta}\right)$ for any $j$, then, in the limit $\widetilde{L}, N=\infty$,

$$
\left|b_{\alpha}^{(j)}\left(\bar{g}_{\|}, \bar{g}_{\perp}, \bar{g}_{4}, \bar{\delta}\right)\right| \leq C\left[\max \left\{\left|\bar{g}_{\|}\right|,\left|\bar{g}_{\perp}\right|,\left|\bar{g}_{4}\right|,|\bar{\delta}|\right\}\right]^{2} \gamma^{\vartheta j}, \quad \alpha=\|, \perp, 4, \delta
$$

The contributions of order $i=0,1$ of the functions $b_{\alpha}^{(j)}(\mathbf{v})$ coincide with the functions $b_{\alpha, i}^{(j)}(\mathbf{v})$ of the Hubbard model, up to the corrections described in (C.17), if we take the limit $L, \beta \rightarrow \infty$. It is easy to see that this implies a difference of order $|\mathbf{v}|^{2} \gamma^{-\left(j-h_{L, \beta}\right)}$, so that we get the bound (2.108).

In we define in a similar way the function $\widetilde{b}^{(j)}\left(g_{\|}, g_{\perp}, g_{4}, \delta\right)$ in terms of $\bar{B}^{(j)}\left(0, G_{\|}, G_{\perp}, G_{4}, \Delta\right)$, in the following paper, see (4.44) of [18, it is also proved that

$$
\left|\widetilde{b}^{(j)}\left(g_{\|}, g_{\perp}, g_{4}, \delta\right)\right| \leq C\left[\max \left\{\left|g_{\|}\right|,\left|g_{\perp}\right|,\left|g_{4}\right|,|\delta|\right\}\right]^{2} \gamma^{\vartheta j}
$$

which implies (2.165).

Note that, in order to prove (2.108), which is a crucial ingredient in the proof of the boundedness of the flow of the spin-symmetric Hubbard model, we need information from a non spin symmetric model; in fact, we have derived (2.108) from the model (C.1) with $g_{\perp} \neq g_{\|}$and $g_{1, \perp}=0$.

Acknowledgements G.B. and V.M. acknowledge the financial support of MIUR, PRIN 2008. V.M. gratefully acknowledges also the financial support from the ERC Starting Grant CoMBoS239694 .

\section{References}

[1] Feldman, Knörrer J.H., and Trubowitz E. A two dimensional fermi liquid. Comm. Math. Phys, 247:1-319, 2004.

[2] A. Giuliani and V. Mastropietro. The 2D Hubbard model on the honeycomb lattice. Comm. Math. Phys., 293:301-346, 2010.

[3] P.W. Anderson. The Theory of Superconductivity in the High-Tc Cuprate Superconductors. Princeton University Press, Princeton, 1997.

[4] F.D.M. Haldane. General relation of correlarion exponents and application to the anisotropic $\mathrm{S}=1 / 2$ Heisenberg chain. Phys. Rev. Lett., 45:1358-1362, 1980.

[5] L.P. Kadanoff and F.J. Wegner. Some critical properties of the Eight-Vertex Model. Phys. Rev. B, 4:3989-3993, 1971.

[6] A. Luther and I. Peschel. Calculation of critical exponents in two dimensions from quantum field theory in one dimension. Phys. Rev. B, 12:3908-3917, 1975.

[7] D. Mattis and E.H. Lieb. Exact solution of a many fermion system and its associated boson field. J. Math. Phys., 6:304-3129, 1965.

[8] F.D.M. Haldane. Luttinger liquid theory of one-dimensional quantum fluids: I. properties of the Luttinger model and their extension to the general 1D interacting spinless Fermi gas. J. Phys. C, 14:2585-2609, 1981. 
[9] J. Sólyom. The Fermi gas model of one-dimensional conductors. Adv. Phys., 28:201-303, 1979.

[10] R.G. Pereira, S.R. White, and I. Affleck. Spectral function of spinless fermions on a onedimensional lattice. Phys. Rev. B, 79, 2009.

[11] G. Benfatto and V. Mastropietro. On the density-density critical indices in interacting Fermi systems. Comm. Math. Phys., 231:97-134, 2002.

[12] G. Benfatto and V. Mastropietro. Ward identities and chiral anomaly in the Luttinger liquid. Comm. Math. Phys., 258:609-655, 2005.

[13] G. Benfatto, G. Gallavotti, A. Procacci, and B. Scoppola. Beta functions and Schwinger functions for a many fermions system in one dimension. Comm. Math. Phys., 160:93-171, 1994.

[14] G. Benfatto and V. Mastropietro. Universality relations in non-solvable quantum spin chains. J. Stat. Phys., 138:1084-1108, 2010.

[15] G. Benfatto, P. Falco, and V. Mastropietro. Universal relations for nonsolvable statistical models. Phys. Rev. Lett., 104:075701, Feb 2010.

[16] T. Giamarchi. Quantum Physics in one dimension. Oxford University Press, 2004.

[17] A.M. Tsvelik. Quantum Field Theory of Condensed Matter Physics. Cambridge University Press, 2007.

[18] G. Benfatto, P. Falco, and V. Mastropietro. Universality of one-dimensional Fermi systems, II. The Luttinger liquid structure. preprint, 2013.

[19] E.H. Lieb and F.Y. Wu. Absence of Mott transition in the 1D Hubbard model. Phys. Rev. Lett., 20:1445-1449, 1968.

[20] D. Mattis. Band theory of magnetism in metals in context of exactly soluble models. Physics, 1:183-193, 1964.

[21] G. Benfatto and V. Mastropietro. Renormalization group, hidden symmetries and approximate Ward identities in the $X Y Z$ model. Rev. Math. Phys., 13:1323-1435, 2001.

[22] G. Benfatto, P. Falco, and V. Mastropietro. Functional integral construction of the massive Thirring model: verification of axioms and massless limit. Comm. Math. Phys., 273:67-118, 2007.

[23] David C. Brydges. A short course on cluster expansions. In Phénomènes critiques, systèmes aléatoires, théories de jauge, (Les Houches, 1984), pages 129-183. North-Holland, Amsterdam, 1986.

[24] A. Lesniewski. Effective action for the Yukawa 2 quantum field theory. Comm. Math. Phys., 108:437-467, 1987.

[25] V. Mastropietro. Rigorous proof of Luttinger liquid behavior in the 1d Hubbard model. $J$. Stat. Phys., 121:373-432, 2005.

[26] A. Giuliani and V. Mastropietro. Anomalous universality in the anisotropic Ashkin-Teller model. Comm. Math. Phys., 256:681-735, 2005.

[27] V. Mastropietro. The absence of logarithmic divergences in the spin and charge density correlations of the 1d Hubbard model. J. Phys. A, 40:3347-3368, 2007.

[28] G. Hardy. Divergent series. Oxford University Press, London, 1949. 